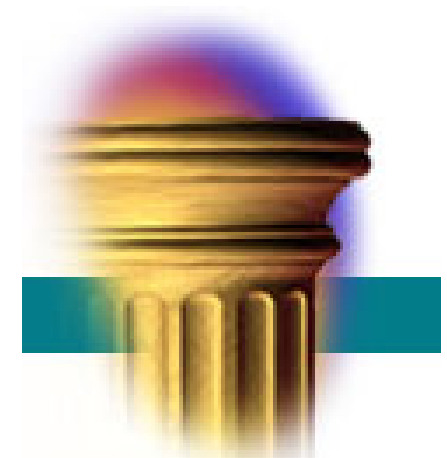

0
0
0
0
0
0
0
0

Identification of Models of the Labor Market

Eric French and Christopher Taber

\author{
REVISED \\ September 23, 2010 \\ WP 2010-08
}




\title{
IDENTIFICATION OF MODELS OF THE LABOR MARKET
}

\author{
Eric French \\ Federal Reserve Bank of Chicago \\ Christopher Taber ${ }^{1}$ \\ Department of Economics \\ University of Wisconsin-Madison \\ and NBER
}

September 23, 2010

\footnotetext{
${ }^{1}$ We thank Pedro Carneiro, Bruce Hansen, John Kennan, Salvador Navarro, Jim Walker, and students in Taber's 2010 Economics 751 class for comments. We also thank Zach Seeskin and David Benson for excellent research assistance. The opinions and conclusions are solely those of the authors, and should not be construed as representing the opinions of the Federal Reserve System.
} 


\begin{abstract}
This chapter discusses identification of common selection models of the labor market. We start with the classic Roy model and show how it can be identified with exclusion restrictions. We then extend the argument to the generalized Roy model, treatment effect models, duration models, search models, and dynamic discrete choice models. In all cases, key ingredients for identification are exclusion restrictions and support conditions.
\end{abstract}

Key words: Identification, Roy model, discrete choice, selection, treatment effects JEL codes: C14, C51, J22, J24 


\section{Introduction}

This chapter discusses identification of common selection models of the labor market. We are primarily concerned with nonparametric identification. We view nonparametric identification as important for the following reasons.

First, recent advances in computer power, more widespread use of large data sets, and better methods mean that estimation of increasingly flexible functional forms is possible. Flexible functional forms should be encouraged. The functional form and distributional assumptions used in much applied work rarely come from the theory. Instead, they come from convenience. Furthermore, they are often not innocuous. ${ }^{1}$

Second, the process of thinking about nonparametric identification is useful input into applied work. It is helpful to an applied researcher both in informing her about which type of data would be ideal and which aspects of the model she might have some hope of estimating. If a feature of the model is not nonparametrically identified, then one knows it cannot be identified directly from the data. Some additional type of functional form assumption must be made. As a result, readers of empirical papers are often skeptical of the results in cases in which the model is not nonparametrically identified.

Third, identification is an important part of a proof of consistency of a nonparametric estimator.

However, we acknowledge the following limitation of focusing on nonparametric identification. With any finite data set, an empirical researcher can almost never be completely nonparametric. Some aspects of the data that might be formally identified could never be estimated with any reasonable level of precision. Instead, estimators are usually only nonparametric in the sense that one allows the flexibility of the model to grow with the sample size. A nice example of this is Sieve estimators in which one estimates finite parameter models but the number of parameters gets large with the data set. An example would be approximating a function by a polynomial and letting the degree of the polynomial get large as the sample size increases. However, in that case one still must verify that the model is nonparametrically identified in

\footnotetext{
${ }^{1}$ A classic reference on this is Lalonde (1986) who shows that parametric models cannot replicate the results of an experiment. Below we present an example on Catholic Schools from Altonji, Elder, and Taber (2005a) suggesting that parametric assumptions drive the empirical estimates.
} 
order to show that the model is consistent. One must also construct standard errors appropriately. In this chapter we do not consider the purely statistical aspects of nonparametric estimation such as calculation of standard errors. This is a very large topic within econometrics. ${ }^{2}$

The key issue in identification of most models of the labor market is the selection problem. For example, individuals are typically not randomly assigned to jobs. With this general goal in mind we begin with the simplest and most fundamental selection model in labor economics, the Roy (1951) model. We go into some detail to explain Heckman and Honoré's (1990) results on identification of this model. A nice aspect of identification of the Roy model is that the basic methodology used in this case can be extended to show identification of other labor models. We spend the rest of the chapter showing how this basic intuition can be used in a wide variety of labor market models. Specifically we cover identification in the generalized Roy model, treatment effect models, the competing risk model, search models, and forward looking dynamic models. While we are clearly not covering all models in labor economics, we hope the ideas are presented in a way that the similarities in the basic models can be seen and can be extended by the reader to alternative frameworks.

The plan of this chapter is specifically as follows. Section 2 discusses some econometric preliminaries. We consider the Roy model in section 3, generalize this to the Generalized Roy model in section 4, and then use the model to think about identification of treatment effects in section 5 . In section 6 we consider duration models and search models and then consider estimation of dynamic discrete choice models in section 7 . Finally in section 8 we offer some concluding thoughts.

\section{Econometric Preliminaries}

\section{$2.1 \quad$ Notation}

Throughout this chapter we use capital letters with $i$ subscripts to denote random variables and small letters without $i$ subscripts to denote possible outcomes of that random variable. We will also try to be explicit throughout this chapter in denoting

\footnotetext{
${ }^{2}$ See Chen (2007) for discussion of Sieve estimators, including standard error calculation.
} 
conditioning. Thus, for example, we will use the notation

$$
E\left(Y_{i} \mid X_{i}=x\right)
$$

to denote the expected value of outcome $Y_{i}$ conditional on the regressor variable $X_{i}$ being equal to some realization $x$.

\subsection{Identification}

The word "identification" has come to mean different things to different labor economists. Here, we use a formal econometrics definition of identification. Consider two different models that lead to data generating processes. If the data generated by these two models have exactly the same distribution then the two models are not separately identified from each other. However, if any two different model specifications lead to different data distributions, the two specifications are separately identified. We give a more precise definition below. Our definition of identification is based on some of the notation and set up of Matzkin's (2007) following an exposition based on Shaikh (2010).

Let $P$ denote the true distribution of the observed data $X$. An econometric model defines a data generating process. We assume that the model is specified up to an unknown vector $\theta$ of parameters, functions and distribution functions. This is known to lie in space $\Theta$. Within the class of models, the element $\theta \in \Theta$ determines the distribution of the data that is observable to the researcher $P_{\theta}$. Notice that identification is fundamentally data dependent. With a richer data set, the distribution $P_{\theta}$ would be a different object.

Let $\mathcal{P}$ be the set of all possible distributions that could be generated by the class of models we consider (i.e. $\mathcal{P} \equiv\left\{P_{\theta}: \theta \in \Theta\right\}$ ). We assume that the model is correctly specified which means that $P \in \mathcal{P}$. The identified set is defined as

$$
\Theta(P) \equiv\left\{\theta \in \Theta: P_{\theta}=P\right\}
$$

This is the set of possible $\theta$ that could have generated data that has distribution $P$. By assuming that $P \in \mathcal{P}$ we have assumed that our model is correctly specified so this set is not empty. We say that $\theta$ is identified if $\Theta(P)$ is a singleton for all $P \in \mathcal{P}$.

The question we seek to answer here is under what conditions is it possible to learn about $\theta$ (or some feature of $\theta$ ) from the distribution of the observed data $P$. 
Our interest is not always to identify the full data generating process. Often we are interested in only a subset of the model, or a particular outcome from it. Specifically, our goal may be to identify

$$
\psi=\Psi(\theta)
$$

where $\Psi$ is a known function. For example in a regression model $Y_{i}=X_{i}^{\prime} \beta+u_{i}$, the feature of interest is typically the regression coefficients. In this case $\Psi$ would take the trivial form

$$
\Psi(\theta)=\beta
$$

However, this notation allows for more general cases in which we might be interested in identifying specific aspects of the model. For example, if our interest is in identifying the covariance between $X$ and $Y$ in the case of the linear regression model, we do not need to know $\theta$ per se, but rather a transformation of these parameters. That is we could be interested in

$$
\Psi(\theta)=\operatorname{Cov}\left(X_{i}, Y_{i}\right)
$$

We could also be interested in a forecast of the model such as

$$
\Psi(\theta)=x^{\prime} \beta
$$

for some specific $x$. The distinction between identification of features of the model as opposed to the full model is important as in many cases the full model is not identified, but the key feature of interest is identified.

To think about identification of $\psi$ we define

$$
\Psi(\Theta(P))=\{\Psi(\theta): \theta \in \Theta(P)\}
$$

That is, it is the set of possible values of $\psi$ that are consistent with the data distribution $P$. We say that $\psi$ is identified if $\Psi(\Theta(P))$ is a singleton.

As an example consider the standard regression model with two regressors:

$$
Y_{i}=\beta_{0}+\beta_{1} X_{1 i}+\beta_{2} X_{2 i}+\varepsilon_{i}
$$

with $E\left(\varepsilon_{i} \mid X_{i}=x\right)=0$ for any value $x$ (where $\left.X_{i}=\left(X_{1 i}, X_{2 i}\right)\right)$. In this case $\theta=\left(\beta, F_{X, \varepsilon}\right)$ where $F_{X, \varepsilon}$ is the joint distribution of $\left(X_{1 i}, X_{2 i}, \varepsilon_{i}\right)$ and $\beta=\left(\beta_{0}, \beta_{1}, \beta_{2}\right)$. One would write $\Theta$ as $\mathcal{B} \times \mathcal{F}_{X, \varepsilon}$ where $\mathcal{B}$ is the parameter space for $\beta$ and $\mathcal{F}_{X, \varepsilon}$ is the 
space of joint distributions between $X_{i}$ and $\varepsilon_{i}$ that satisfy $E\left(\varepsilon_{i} \mid X_{i}=x\right)=0$ for all $x$. Since the data here is represented by $\left(X_{1 i}, X_{2 i}, Y_{i}\right), P_{\theta}$ represents the joint distribution of $\left(X_{1 i}, X_{2 i}, Y_{i}\right)$. Given knowledge of $\beta$ and $F_{X, \varepsilon}$ we know the data generating process and thus we know $P_{\theta}$.

To focus ideas suppose we are interested in identifying $\beta$ (i.e. $\Psi\left(\beta, F_{X, \varepsilon}\right)=\beta$ ) in regression model (2.1) above. Let the true value of the data generating process be $\theta^{*}=$ $\left(\beta^{*}, F_{X, \varepsilon}^{*}\right)$ so that by definition $P_{\theta^{*}}=P$. In this case $\Theta(P) \equiv\left\{\left(\beta, F_{X, \varepsilon}\right) \in \mathcal{B} \times \mathcal{F}_{X, \varepsilon}: P_{\beta, F_{x, \varepsilon}}=P\right\}$, that is it is the set of $\left(\beta, F_{X, \varepsilon}\right)$ that would lead our data $\left(X_{i}, Y_{i}\right)$ to have distribution $P$. In this case $\Psi(\Theta(P))$ is the set of values of $\beta$ in this set (i.e. $\Psi(\Theta(P))=$ $\left\{\beta:\left(\beta, F_{X, \varepsilon}\right) \in \Theta(P)\right.$ for some $\left.\left.F_{X, \varepsilon} \in \mathcal{F}_{X, \varepsilon}\right\}\right)$.

In the case of 2 covariates, we know the model is identified as long as $X_{1 i}$ and $X_{2 i}$ are not degenerate and not collinear. To see how this definition of identification applies to this model, note that for any $\beta^{*} \neq \beta$ the lack of perfect multicollinearity means that we can always find values of $\left(x_{1}, x_{2}\right)$ for which

$$
\beta_{0}+\beta_{1} x_{1}+\beta_{2} x_{2} \neq \beta_{0}^{*}+\beta_{1}^{*} x_{1}+\beta_{2}^{*} x_{2} .
$$

Since $E\left(Y_{i} \mid X_{i}=x\right)$ is one aspect of the joint distribution of $P_{\theta}$, it must be the case that when $\beta^{*} \neq \beta, P_{\theta} \neq P$. Since this is true for any value of $\beta \neq \beta^{*}$, then $\Psi(\Theta(P))$ must be the singleton $\beta^{*}$.

However, consider the well known case of perfect multicollinearity in which the model is not identified. In particular suppose that

$$
X_{1 i}+X_{2 i}=1
$$

For the true value of $\beta^{*}=\left(\beta_{0}^{*}, \beta_{1}^{*}, \beta_{2}^{*}\right)$ consider some other value $\widetilde{\beta}=\left(\beta_{0}^{*}+\beta_{2}^{*}, \beta_{1}^{*}-\right.$ $\left.\beta_{2}^{*}, 0\right)$. Then for any $x$,

$$
\begin{aligned}
E\left(Y_{i} \mid X_{i}=x\right) & =\beta_{0}^{*}+\beta_{1}^{*} x_{1}+\beta_{2}^{*} x_{2} \\
& =\beta_{0}^{*}+\beta_{1}^{*} x_{1}+\beta_{2}^{*}\left(1-x_{1}\right) \\
& =\beta_{0}^{*}+\beta_{2}^{*}+\left(\beta_{1}^{*}-\beta_{2}^{*}\right) x_{1} \\
& =\widetilde{\beta}_{0}+\widetilde{\beta}_{1} x_{1} .
\end{aligned}
$$

If $F_{X, \varepsilon}$ is the same for the two models, then the joint distribution of $\left(Y_{i}, X_{i}\right)$ is the same in the two cases. Thus the identification condition above is violated because with 
$\widetilde{\theta}=\left(\widetilde{\beta}, F_{X, \varepsilon}^{*}\right), P_{\widetilde{\theta}}=P$ and thus $\widetilde{\beta} \in \Psi(\Theta(P))$. Since the true value $\beta^{*} \in \Psi(\Theta(P))$ as well, $\Psi(\Theta(P))$ is not a singleton and thus $\beta$ is not identified.

\subsection{Support}

Another important issue is the support of the data. The simplest definition of support is just the range of the data. When data are discrete, this is the set of values that occur with positive probability. Thus a binary variable that is either zero or one would have support $\{0,1\}$. The result of a die roll has support $\{1,2,3,4,5,6\}$. With continuous variables things get somewhat more complicated. One can think of the support of a random variable as the set of values for which the density is positive. For example, the support of a normal random variable would be the full real line (which we will often refer to as "full support"). The support of a uniform variable on $[0,1]$ is $[0,1]$. The support of an exponential variable would be the positive real line.

This can be somewhat trickier in dealing with outcomes that occur with measure zero. For example one could think of the support of a uniform variable as $[0,1],(0,1],[0,1)$, or $(0,1)$. The distinction between these objects will not be important in what we are doing, but to be formal we will use the Davidson (1994) definition of support. He defines the support of a random variable with distribution $F$ as the set of points at which $F$ is (strictly) increasing. ${ }^{3}$ By this definition, the support of a uniform would be $[0,1]$. We will also use the notation $\operatorname{supp}\left(Y_{i}\right)$ to denote the unconditional support of random variable $Y_{i}$ and $\operatorname{supp}\left(Y_{i} \mid X_{i}=x\right)$ to denote the conditional support.

To see the importance of this concept, consider a simple case of the separable regression model

$$
Y_{i}=g\left(X_{i}\right)+u_{i}
$$

with a single continuous $X_{i}$ variable and $E\left(u_{i} \mid X_{i}=x\right)=0$ for $x \in \operatorname{supp}\left(X_{i}\right)$. In this case we know that

$$
E\left(Y_{i} \mid X_{i}=x\right)=g(x)
$$

Letting $\mathcal{X}$ be the support of $X_{i}$, it is straightforward to see that $g$ is identified on the set $\mathcal{X}$. But $g$ is not identified outside the set $\mathcal{X}$ because the data is completely silent about these values. Thus if $\mathcal{X}=\mathbb{R}, g$ is globally identified. However, if $\mathcal{X}$ only covers a subset

\footnotetext{
${ }^{3} \mathrm{He}$ defines $F$ (strictly) increasing at point $x$ to mean that for any $\varepsilon>0, F(x+\varepsilon)>F(x-\varepsilon)$.
} 
of the real line it is not. For example, one interesting counterfactual is the change in the expected value of $Y_{i}$ if $X_{i}$ were increased by $\delta: E\left(g\left(X_{i}+\delta\right)\right)$. If $\mathcal{X}=\mathbb{R}$ this is trivially identified, but if the support of $X_{i}$ were bounded from above, this would no longer be the case. That is, if the supremum of $\mathcal{X}$ is $\bar{x}<\infty$, then for any value of $x>\bar{x}-\delta$, $g(x+\delta)$ is not identified and thus the unconditional expected value of $g\left(X_{i}+\delta\right)$ is not identified either. This is just a restatement of the well known fact that one cannot project out of the data unless one makes functional form assumptions. Our point here is that support assumptions are very important in nonparametric identification results. One can only identify $g$ over the range of plausible values of $X_{i}$ if $X_{i}$ has full support. For this reason, we will often make strong support condition assumptions. This also helps illuminate the tradeoff between functional form assumptions and flexibility. In order to project off the support of the data in a simple regression model one needs to use some functional form assumption. The same is true for selection models.

\subsection{Continuity}

There is one complication that we need to deal with throughout. It is not a terribly important issue, but will shape some of our assumptions. Consider again the separable regression model

$$
Y_{i}=g\left(X_{i}\right)+u_{i}
$$

As mentioned above $E\left(Y_{i} \mid X_{i}=x\right)=g(x)$ so it seems trivial to see that $g$ is identified, but that is not quite true. To see the problem, suppose that both $X_{i}$ and $u_{i}$ are standard normals. Consider two different models for $g$,

Model 1:

$$
g(x)= \begin{cases}0 & x<1.4 \\ 1 & x \geq 1.4\end{cases}
$$

versus

Model 2:

$$
g(x)=\left\{\begin{array}{ll}
0 & x \leq 1.4 \\
1 & x>1.4
\end{array} .\right.
$$

These models only differ at the point $x=1.4$, but since $X_{i}$ is normal this is a zero probability event and we could never distinguish between these models because they 
imply the same joint distribution of $\left(X_{i}, Y_{i}\right)$. For the exact same reason it isn't really a concern (except in very special cases such as if one was evaluating a policy in which we would set $X_{i}=1.4$ for everyone). Since this will be an issue throughout this chapter we explain how to deal with it now and use this convention throughout the chapter.

We will make the following assumptions.

Assumption 2.1. $X_{i}$ can be written as $\left(X_{i}^{c}, X_{i}^{d}\right)$ where the elements of $X_{i}^{c}$ are continuously distributed (no point has positive mass), and $X_{i}^{d}$ is distributed discretely (all support points have positive mass).

Assumption 2.2. For any $x^{d} \in \operatorname{supp}\left(X_{i}^{d}\right), g\left(x^{c}, x^{d}\right)$ is almost surely continuous across $x^{c} \in \operatorname{supp}\left(X_{i}^{c} \mid X_{i}^{d}=x^{d}\right)$.

The first part says that we can partition our observables into continuous and discrete ones. One could easily allow for variables that are partially continuous and partially discrete, but this would just make our results more tedious to exposit. The second assumption states that choosing a value of $X$ at which $g$ is discontinuous (in the continuous variables) is a zero probability event.

Theorem 2.1. Under assumptions 2.1 and 2.2 and assuming model 2.2 with $E\left(u_{i} \mid\right.$ $\left.X_{i}=x\right)=0$ for $x \in \operatorname{supp}\left(X_{i}\right), g(x)$ is identified on a set $\mathcal{X}^{*}$ that has measure 1 .

(Proof in Appendix)

The proof just states that $g$ is identified almost everywhere. More specifically it is identified everywhere that it is continuous.

\section{The Roy Model}

The classic model of selection in the labor market is the Roy (1951) model. In the Roy model, workers choose one of two possible occupations: hunting and fishing. They cannot pursue both at the same time. The worker's log wage is $Y_{f i}$ if he fishes and $Y_{h i}$ if he hunts. Workers maximize income so they choose the occupation with higher wage. Thus a worker chooses to fish if $Y_{f i}>Y_{h i}$. The occupation is defined as

$$
J_{i}= \begin{cases}f & \text { if } Y_{f i}>Y_{h i} \\ h & \text { if } Y_{h i} \geq Y_{f i}\end{cases}
$$


and the log wage is defined as

$$
Y_{i}=\max \left\{Y_{f i}, Y_{h i}\right\}
$$

Workers face a simple binary choice: choose the job with the highest wage. This simplicity has led the model to be used in one form or another in a number of important labor market contexts. Many discrete choice models share the Roy model's structure. Examples in labor economics include the choice of whether to continue schooling, what school to attend, what occupation to pursue, whether to join a union, whether to migrate, whether to work, whether to obtain training, and whether to marry.

As mentioned in the introduction, we devote considerable attention to identification of this model. In subsequent sections we generalize these results to other models.

The responsiveness of the supply of fishermen to changes in the price of fish depends critically on the joint distribution of $\left(Y_{f i}, Y_{h i}\right)$. Thus we need to know what a fisherman would have made if he had chosen to hunt. However, we do not observe this but must infer its counterfactual distribution from the data at hand. Our focus is on this selection problem. Specifically, much of this chapter is concerned with the following question: Under what conditions is the joint distribution of $\left(Y_{f i}, Y_{h i}\right)$ identified? We start by considering estimation in a parametric model and then consider nonparametric identification.

Roy (1951) is concerned with how occupational choice affects the aggregate distribution of earnings and makes a series of claims about this relationship. These claims turn out to be true when the distribution of skills in the two occupations is lognormal.

Heckman and Honoré (1990) consider identification of the Roy model (i.e., the joint distribution of $\left.\left(Y_{f i}, Y_{h i}\right)\right)$. They show that there are two methods for identifying the Roy model. The first is through distributional assumptions. The second is through exclusion restrictions. ${ }^{4}$

In order to focus ideas, we use the following case:

$$
\begin{aligned}
& Y_{f i}=g_{f}\left(X_{f i}, X_{0 i}\right)+\varepsilon_{f i} \\
& Y_{h i}=g_{h}\left(X_{h i}, X_{0 i}\right)+\varepsilon_{h i}
\end{aligned}
$$

\footnotetext{
${ }^{4}$ Heckman and Honoré discuss price variation as separate from exclusion restrictions. However, in our framework price changes can be modeled as just one type of exclusion restriction so we do not explicitly discuss price variation.
} 
where the unobservable error terms $\left(\varepsilon_{f i}, \varepsilon_{h i}\right)$ are independent of the observable variables $X_{i}=\left(X_{f i}, X_{h i}, X_{0 i}\right)$ and $Y_{f i}$ and $Y_{h i}$ denote log wages in the fishing and hunting sectors respectively. We distinguish between three types of variables. $X_{0 i}$ influences productivity in both fishing and hunting, $X_{f i}$ influences fishing only, and $X_{h i}$ influences hunting only. The variables $X_{f i}$ and $X_{h i}$ are "exclusion restrictions," and play a very important role in the identification results below. In the context of the Roy model, an exclusion restriction could be a change in the price of rabbits which increases income from hunting, but not from fishing. The notation is general enough to incorporate a model without exclusion restrictions (in which case one or more of the $X_{j i}$ would be empty).

Our version of the Roy framework imposes two strong assumptions. First, that $Y_{j i}$ is separable in $g_{j}\left(X_{j i}, X_{0 i}\right)$ and $\varepsilon_{j i}$ for $j \in\{f, h\}$. Second, we assume that $g_{j}\left(X_{j i}, X_{0 i}\right)$ and $\varepsilon_{j i}$ are independent of one another. Note that independence implies homoskedasticity: the variance of $\varepsilon_{j i}$ cannot depend on $X_{j i}$. There is a large literature looking at various other more flexible specifications and this is discussed thoroughly in Matzkin (2007). It is also trivial to extend this model to allow for a general relationship between $X_{0 i}$ and $\left(\varepsilon_{f i}, \varepsilon_{h i}\right)$ as we discuss in section 3.3 below.

We focus on the separable independent model for two reasons. First, the assumptions of separability and independence have bite beyond a completely general nonparametric relationship. That is, to the extent that they are true, identification is facilitated by these assumptions. Presumably because researchers think these assumptions are approximately true, virtually all empirical research uses these assumptions. Second, despite these strong assumptions, they are obviously much weaker than the standard assumptions that $g$ is linear (i.e. $g_{f}\left(X_{f i}, X_{0 i}\right)=X_{f i}^{\prime} \gamma_{f f}+X_{0 i}^{\prime} \gamma_{0 f}$ and that $\varepsilon_{f i}$ is normally distributed. One approach to writing this chapter would have been to go through all of the many specifications and alternative assumptions. We choose to focus on a single base specification for expositional simplicity.

Heckman and Honoré (1990) first discuss identification of the joint distribution of $\left(Y_{f i}, Y_{h i}\right)$ using distributional assumptions. They show that when one can observe the distribution of wages in both sectors, and assuming $\left(Y_{f i}, Y_{h i}\right)$ is joint normally distributed, then the joint distribution of $\left(Y_{f i}, Y_{h i}\right)$ is identified from a single cross section even without any exclusion restrictions or regressors. To see why, write equations (3.3) 
and (3.4) without regressors (so $g_{f}=\mu_{f}$, the mean of $\left.Y_{f i}\right)$ :

$$
\begin{aligned}
& Y_{f i}=\mu_{f}+\varepsilon_{f i} \\
& Y_{h i}=\mu_{h}+\varepsilon_{h i}
\end{aligned}
$$

where

$$
\left[\begin{array}{c}
\varepsilon_{f i} \\
\varepsilon_{h i}
\end{array}\right]=N\left(\left[\begin{array}{l}
0 \\
0
\end{array}\right],\left[\begin{array}{cc}
\sigma_{f}^{2} & \sigma_{f h} \\
\sigma_{f h} & \sigma_{h}^{2}
\end{array}\right]\right)
$$

Letting

$$
\lambda(\cdot)=\frac{\phi(\cdot)}{\Phi(\cdot)}
$$

(with $\phi$ and $\Phi$ the pdf and cdf of a standard normal),

$$
c=\frac{\mu_{f}-\mu_{h}}{\sqrt{\sigma_{f}^{2}+\sigma_{h}^{2}-2 \sigma_{f h}}},
$$

and for each $j \in\{h, f\}$,

$$
\tau_{j}=\frac{\sigma_{j}^{2}-\sigma_{f h}}{\sqrt{\sigma_{f}^{2}+\sigma_{h}^{2}-2 \sigma_{f h}}} .
$$

One can derive the following conditions from properties of normal random variables found in Heckman and Honoré (1990):

$$
\begin{aligned}
\operatorname{Pr}\left(J_{i}=f\right) & =\Phi(c) \\
E\left(Y_{i} \mid J_{i}=f\right) & =\mu_{f}+\tau_{f} \lambda(c) \\
E\left(Y_{i} \mid J_{i}=h\right) & =\mu_{h}+\tau_{h} \lambda(-c) \\
\operatorname{Var}\left(Y_{i} \mid J_{i}=f\right) & =\sigma_{f}^{2}+\tau_{f}^{2}\left(-\lambda(c) c-\lambda^{2}(c)\right) \\
\operatorname{Var}\left(Y_{i} \mid J_{i}=h\right) & =\sigma_{h}^{2}+\tau_{h}^{2}\left(\lambda(-c) c-\lambda^{2}(-c)\right) \\
E\left(\left[Y_{i}-E\left(Y_{i} \mid J_{i}=f\right)\right]^{3} \mid J_{i}=f\right) & =\tau_{f}^{3} \lambda(c)\left[2 \lambda^{2}(c)+3 c \lambda(c)+c^{2}-1\right] . \\
E\left(\left[Y_{i}-E\left(Y_{i} \mid J_{i}=h\right)\right]^{3} \mid J_{i}=h\right) & =\tau_{h}^{3} \lambda(-c)\left[2 \lambda^{2}(-c)-3 c \lambda(-c)+c^{2}-1\right] .
\end{aligned}
$$

This gives us seven equations in the five unknowns $\mu_{f}, \mu_{h}, \sigma_{f}^{2}, \sigma_{h}^{2}$, and $\sigma_{f h}$. It is straightforward to show that the five parameters can be identified from this system of equations. 
However, Theorems 7 and 8 of Heckman and Honoré (1990) show that when one relaxes the log normality assumption, without exclusion restrictions in the outcome equation, the model is no longer identified. This is true despite the strong assumption of agent income maximization. This result is not particularly surprising in the sense that our goal is to estimate a full joint distribution of a two dimensional object $\left(Y_{f i}, Y_{h i}\right)$, but all we can observe is two one dimensional distributions (wages conditional on job choice). Since there is no information in the data about the wage that a fisherman may have received as a hunter, one cannot identify this joint distribution. In fact, Theorem 7 of Heckman and Honoré (1990) states that we can never distinguish the actual model from an alternative model in which skills are independent of each other.

\subsection{Estimation of the Normal Linear Labor Supply model}

It is often the case that we only observe wages in one sector. For example, when estimating models of participation in the labor force, the wage is observed only if the individual works. We can map this into our model by associating working with "fishing" and not working with "hunting." That is, we let $Y_{f i}$ denote income if working and let $Y_{h i}$ denote the value of not working. ${ }^{5}$

But there are other examples in which we observe the wage in only one sector. For example, in many data sets we do not observe wages of workers in the black market sector. Another example is return immigration in which we know when a worker leaves the data to return to their home country, but we do not observe that wage.

In Section 3.2 we discuss identification of the nonparametric version of the model. However, it turns out that identification of the more complicated model is quite similar to estimation of the model with normally distributed errors. Thus we review this in detail before discussing the nonparametric model. We also remark that providing a consistent estimator also provides a constructive proof of identification, so one can also interpret these results as (informally) showing identification in the normal model. The

\footnotetext{
${ }^{5}$ There are two common participation models. The first is the home production model in which the individual chooses between home and market production. The second is the labor supply model in which the individual chooses between market production and leisure. In practice the two types of models tend to be similar and some might argue the distinction is semantic. In a model of home production, $Y_{h i}$ is the (unobserved) gain from home production. In a model of labor supply, $Y_{h i}$ is the leisure value of not working.
} 
model is similar to Willis and Rosen's (1979) Roy Model of educational choices or Lee's (1978) model of union status and the empirical approach is analogous. We assume that

$$
\begin{gathered}
Y_{f i}=X_{f i}^{\prime} \gamma_{f f}+X_{0 i}^{\prime} \gamma_{0 f}+\varepsilon_{f i} \\
Y_{h i}=X_{h i}^{\prime} \gamma_{h h}+X_{0 i}^{\prime} \gamma_{0 h}+\varepsilon_{h i} \\
{\left[\begin{array}{c}
\varepsilon_{f i} \\
\varepsilon_{h i}
\end{array}\right]=N\left(\left[\begin{array}{l}
0 \\
0
\end{array}\right],\left[\begin{array}{cc}
\sigma_{f}^{2} & \sigma_{f h} \\
\sigma_{f h} & \sigma_{h}^{2}
\end{array}\right]\right) .}
\end{gathered}
$$

In a labor supply model where $f$ represents market work, $Y_{f i}$ is the market wage which will be observed for workers only. $Y_{h i}$, the pecuniary value of not working, is never observed in the data. Keane, Todd, and Wolpin's (this volume) example of the static model of a married woman's labor force participation is similar.

One could simply estimate this model by maximum likelihood. However we discuss a more traditional four step method to illustrate how the parametric model is identified. This four step process will be analogous to the more complicated nonparametric identification below. Step 1 is a "reduced form probit" of occupational choices as a function of all covariates in the model. Step 2 estimates the wage equations by controlling for selection as in the second step of a Heckman Two step (Heckman, 1979). Step 3 uses the coefficients of the wage equations and plugs these back into a probit equation to estimate a "structural probit." Step 4 shows identification of the remaining elements of the variance-covariance matrix of the residuals.

\section{Step 1: Estimation of Choice Model}

The probability of choosing fishing (i.e., work) is:

$$
\begin{aligned}
\operatorname{Pr}\left(J_{i}=f \mid X_{i}=x\right) & =\operatorname{Pr}\left(Y_{f i}>Y_{h i} \mid X_{i}=x\right) \\
& =\operatorname{Pr}\left(x_{f}^{\prime} \gamma_{f f}+x_{0}^{\prime} \gamma_{0 f}+\varepsilon_{f i}>x_{0}^{\prime} \gamma_{0 h}+x_{h}^{\prime} \gamma_{h h}+\varepsilon_{h i}\right) \\
& =\operatorname{Pr}\left(x_{f}^{\prime} \gamma_{f f}-x_{h}^{\prime} \gamma_{h h}+x_{0}^{\prime}\left(\gamma_{0 f}-\gamma_{0 h}\right)>\varepsilon_{h i}-\varepsilon_{f i}\right) \\
& =\Phi\left(\frac{x_{f}^{\prime} \gamma_{f f}-x_{h}^{\prime} \gamma_{h h}+x_{0}^{\prime}\left(\gamma_{0 f}-\gamma_{0 h}\right)}{\sigma^{*}}\right) \\
& =\Phi\left(x^{\prime} \gamma^{*}\right)
\end{aligned}
$$

where $\Phi$ is the cdf of a standard normal, $\sigma^{*}$ is the standard deviation of $\left(\varepsilon_{h i}-\varepsilon_{f i}\right)$, and

$$
\gamma^{*} \equiv\left(\frac{\gamma_{f f}}{\sigma^{*}}, \frac{-\gamma_{h h}}{\sigma^{*}}, \frac{\gamma_{0 f}-\gamma_{0 h}}{\sigma^{*}}\right)
$$


This is referred to as the "reduced form model" as it is a reduced form in the classical sense: the parameters are a known function of the underlying structural parameters. It can be estimated by maximum likelihood as a probit model. Let $\widehat{\gamma^{*}}$ represent the estimated parameter vector. This is all that can be learned from the choice data alone. We need further information to identify $\sigma^{*}$ and to separate $\gamma_{0 f}$ from $\gamma_{0 h}$.

\section{Step 2: Estimating the Wage Equation}

This is essentially the second stage of a Heckman (1979) two step. To review the idea behind it, let

$$
\varepsilon_{i}^{*}=\frac{\varepsilon_{h i}-\varepsilon_{f i}}{\sigma^{*}}
$$

Then consider the regression

$$
\varepsilon_{f i}=\tau \varepsilon_{i}^{*}+\zeta_{i}
$$

where $\operatorname{cov}\left(\varepsilon_{i}^{*}, \zeta_{i}\right)=0$ (by definition of regression) and thus:

$$
\begin{aligned}
\tau & =\frac{\operatorname{cov}\left(\varepsilon_{f i}, \varepsilon_{i}^{*}\right)}{\operatorname{var}\left(\varepsilon_{i}^{*}\right)} \\
& =E\left[\varepsilon_{f i}\left(\frac{\varepsilon_{h i}-\varepsilon_{f i}}{\sigma^{*}}\right)\right] \\
& =\frac{\sigma_{f h}-\sigma_{f}^{2}}{\sigma^{*}}
\end{aligned}
$$

The wage of those who choose to work is

$$
\begin{aligned}
E\left(Y_{f i} \mid J_{i}=f, X_{i}=x\right) & =x_{f}^{\prime} \gamma_{f f}+x_{0}^{\prime} \gamma_{0 f}+E\left(\varepsilon_{f i} \mid J_{i}=f, X_{i}=x\right) \\
& =x_{f}^{\prime} \gamma_{f f}+x_{0}^{\prime} \gamma_{0 f}+E\left(\tau \varepsilon_{i}^{*}+\zeta_{i} \mid \varepsilon_{i}^{*} \leq x^{\prime} \gamma^{*}\right) \\
& =x_{f}^{\prime} \gamma_{f f}+x_{0}^{\prime} \gamma_{0 f}+\tau E\left(\varepsilon_{i}^{*} \mid \varepsilon_{i}^{*} \leq x^{\prime} \gamma^{*}\right) \\
& =x_{f}^{\prime} \gamma_{f f}+x_{0}^{\prime} \gamma_{0 f}-\tau \lambda\left(x^{\prime} \gamma^{*}\right) .
\end{aligned}
$$

Showing that $E\left(\varepsilon_{i}^{*} \mid \varepsilon_{i}^{*} \leq x^{\prime} \gamma^{*}\right)=-\lambda\left(x^{\prime} \gamma^{*}\right)$ is a fairly straightforward integration problem and is well known. Because equation (3.6) is a conditional expectation function, OLS regression of $Y_{i}$ on $X_{0 i}, X_{f i}$, and $\lambda\left(X_{i}^{\prime} \widehat{\gamma^{*}}\right)$ gives consistent estimates of $\gamma_{f f}, \gamma_{0 f}$, and $\tau . \widehat{\gamma}^{*}$ is the value of $\gamma^{*}$ estimated in equation (3.5).

Note that we do not require an exclusion restriction. Since $\lambda$ is a nonlinear function, but $g_{f}$ is linear, this model is identified. However, without an exclusion restriction, identification is purely through functional form. When we consider a nonparametric 
version of the model below, exclusion restrictions are necessary. We discuss this issue in section 3.2 .

\section{Step 3: The Structural Probit}

Our next goal is to estimate $\gamma_{0 h}$ and $\gamma_{h h}$. In Step 1 we obtained consistent estimates

of $\gamma^{*} \equiv\left(\frac{\gamma_{0 f}-\gamma_{0 h}}{\sigma^{*}}, \frac{\gamma_{f f}}{\sigma^{*}}, \frac{-\gamma_{h h}}{\sigma^{*}}\right)$ and in Step 2 we obtained consistent estimates of $\gamma_{0 f}$ and $\gamma_{f f}$

When there is only one exclusion restriction (i.e. $\gamma_{f f}$ is a scalar), identification proceeds as follows. Because we identified $\gamma_{f f}$ in Step 2 and $\gamma_{f f} / \sigma^{*}$ in Step 1, we can identify $\sigma^{*}$. Once $\sigma^{*}$ is identified, it is easy to see how to identify $\gamma_{h h}$ (because $\frac{-\gamma_{h h}}{\sigma^{*}}$ is identified) and $\gamma_{0 h}$ (because $\frac{\gamma_{0 f}-\gamma_{0 h}}{\sigma^{*}}$ and $\gamma_{0 f}$ are identified).

In terms of estimation of these objects, if there is more than one exclusion restriction the model is over-identified. If we have two exclusion restrictions, $\gamma_{f f}$ and $\gamma_{f f} / \sigma^{*}$ are both $2 \times 1$ vectors, and thus we wind up with 2 consistent estimates of $\sigma^{*}$. The most standard way of solving this model is by estimating the "structural probit:"

$$
\operatorname{Pr}\left(J_{i}=f \mid X_{i}=x\right)=\Phi\left(\frac{1}{\sigma^{*}}\left(x_{f}^{\prime} \widehat{\gamma_{f f}}+x_{0}^{\prime} \widehat{\gamma_{0 f}}\right)-x_{h}^{\prime} \frac{\gamma_{h h}}{\sigma^{*}}-x_{0}^{\prime} \frac{\gamma_{0 h}}{\sigma^{*}}\right) .
$$

That is, one just runs a probit of $J_{i}$ on $\left(X_{f i}^{\prime} \widehat{\gamma_{f f}}+X_{0 i}^{\prime} \widehat{\gamma_{0 f}}\right), X_{0 i}$, and $X_{h i}$ where $\widehat{\gamma_{f f}}$ and $\widehat{\gamma_{0 f}}$ are our our estimates of $\gamma_{f f}$ and $\gamma_{0 f}$.

Step 3 is essential if our goal is to estimate the labor supply equation. If we are only interested in controlling for selection to obtain consistent estimates of the wage equation, we do not need to worry about the structural probit. However, notice that

$$
\frac{\partial \operatorname{Pr}\left(J_{i}=f \mid X_{i}=x\right)}{\partial Y_{f i}}=\frac{1}{\sigma^{*}} \phi\left(x^{\prime} \gamma^{*}\right) .
$$

and thus the labor supply elasticity is:

$\frac{\partial \log \left[\operatorname{Pr}\left(J_{i}=f \mid X_{i}=x\right)\right]}{\partial Y_{f i}}=\frac{\partial \operatorname{Pr}\left(J_{i}=f \mid X_{i}=x\right)}{\partial Y_{f i}} \frac{1}{\operatorname{Pr}\left(J_{i}=f \mid X_{i}=x\right)}=\frac{1}{\sigma^{*}} \frac{\phi\left(x^{\prime} \gamma^{*}\right)}{\Phi\left(x^{\prime} \gamma^{*}\right)}$, where, as before, $Y_{f i}$ is the log of income if working. Thus knowledge of $\sigma^{*}$ is essential for identifying the effects of wages on participation.

One could not estimate the structural probit without the exclusion restriction $X_{f i}$ as the first two components of the probit in equation (3.7) would be perfectly collinear. For any $\sigma^{*}>0$ we could find a value of $\gamma_{0 h}$ and $\gamma_{h h}$ to that delivers the same choice probabilities. Furthermore, if these parameters were not identified, the elasticity of labor supply with respect to wages would not be identified either. 


\section{Step 4: Estimation of the Variance Matrix of the Residuals}

Lastly, we identify all the components of $\Sigma,\left(\sigma_{f}^{2}, \sigma_{h}^{2}, \sigma_{f h}\right)$ as follows. We have described

how to obtain consistent estimates of $\sigma^{*}=\sqrt{\sigma_{f}^{2}+\sigma_{h}^{2}-2 \sigma_{f h}}$ and $\tau=\frac{\sigma_{f h}-\sigma_{f}^{2}}{\sigma^{*}}$. This gives us two equations in three parameters. We can obtain the final equation by using the variance of the residual in the selection model since

$$
\operatorname{Var}\left(\varepsilon_{f i} \mid J_{i}=f, X_{i}=x\right)=\sigma_{f}^{2}+\tau^{2}\left[-\lambda\left(x^{\prime} \gamma^{*}\right) x^{\prime} \gamma^{*}-\lambda^{2}\left(x^{\prime} \gamma^{*}\right)\right]
$$

Let $i=1, . ., N_{f}$ index the set of individuals who choose $J_{i}=f$ and $\widehat{\varepsilon_{f i}}$ is the residual $Y_{f i}-X_{f i}^{\prime} \widehat{\gamma_{f f}}-X_{0 i}^{\prime} \widehat{\gamma_{0 f}}$ Using "hats" to denote estimators we can estimate the model as

$$
\begin{aligned}
\widehat{\sigma_{f}^{2}} & =\frac{1}{N_{f}} \sum_{i=1}^{N_{f}}\left(\widehat{\varepsilon_{f i}}+\tau \lambda\left(X_{i}^{\prime}{\widehat{\gamma^{*}}}^{*}\right)^{2}-\widehat{\tau}^{2}\left(-\lambda\left(X_{i}^{\prime} \widehat{\gamma}^{*}\right) X_{i}^{\prime}{\widehat{\gamma^{*}}}^{2} \lambda^{2}\left(X_{i}^{\prime} \widehat{\gamma}^{*}\right)\right)\right. \\
\widehat{\sigma_{f h}} & =\widehat{\sigma_{f}^{2}}-\widehat{\tau} \widehat{\sigma}^{*} \\
{\widehat{\sigma_{h}}}^{2} & ={\widehat{\sigma^{*}}}^{2}-{\widehat{\sigma_{f}}}^{2}+2 \widehat{\sigma_{f h}}
\end{aligned}
$$

\subsection{Identification of the Roy Model: the Non-Parametric Ap- proach}

Although the parametric case with exclusion restrictions is more commonly known, the model in the previous section is still identified non-parametrically if the researcher is willing to impose stronger support conditions on the observable variables. Heckman and Honoré (1990, Theorem 12) provide conditions under which one can identify the model nonparametrically using exclusion restrictions. We present this case below.

Assumption 3.1. $\left(\varepsilon_{f i}, \varepsilon_{h i}\right)$ is continuously distributed with distribution function $G$, support $\mathbb{R}^{2}$, and is independent of $X_{i}$. The marginal distributions of $\varepsilon_{f i}$ and $\varepsilon_{f i}-\varepsilon_{h i}$ have medians equal to zero.

Assumption 3.2. $\operatorname{supp}\left(g_{f}\left(X_{f i}, x_{0}\right), g_{h}\left(X_{h i}, x_{0}\right)\right)=\mathbb{R}^{2}$ for all $x_{0} \in \operatorname{supp}\left(X_{0 i}\right)$.

Assumption 3.2 is crucial for identification. It states that for any value of $g_{h}\left(x_{h}, x_{0}\right)$, $g_{f}\left(X_{f i}, x_{0}\right)$ varies across the full real line and for any value of $g_{f}\left(x_{f}, x_{0}\right), g_{h}\left(X_{h i}, x_{0}\right)$ varies across the full real line. This means that we can condition on a set of variables for which the probability of being a hunter (i.e. $\left.\operatorname{Pr}\left(J_{i}=h \mid X_{i}=x\right)\right)$ is arbitrarily close to 1 . This is clearly a very strong assumption that we will discuss further.

We need the following two assumptions for the reasons discussed in section 2.4. 
Assumption 3.3. $X_{i}=\left(X_{f i}, X_{h i}, X_{0 i}\right)$ can be written as $\left(X_{f i}^{c}, X_{f i}^{d}, X_{h i}^{c}, X_{h i}^{d}, X_{0 i}^{c}, X_{0 i}^{d}\right)$ where the elements of $\left(X_{f i}^{c}, X_{h i}^{c}, X_{0 i}^{c}\right)$ are continuously distributed (no point has positive mass), and $\left(X_{f i}^{d}, X_{h i}^{d}, X_{0 i}^{d}\right)$ is distributed discretely (all support points have positive mass).

Assumption 3.4. For any $\left(x_{f}^{d}, x_{h}^{d}, x_{0}^{d}\right) \in \operatorname{supp}\left(X_{f i}^{d}, X_{h i}^{d}, X_{0 i}^{d}\right), g_{f}\left(x_{f}^{c}, x_{f}^{d}, x_{0}^{c}, x_{0}^{d}\right)$ and $g_{h}\left(x_{h}^{c}, x_{h}^{d}, x_{0}^{c}, x_{0}^{d}\right)$ are almost surely continuous across $x^{c} \in \operatorname{supp}\left(X_{i}^{c} \mid X_{i}^{d}=x^{d}\right)$.

Under these assumptions we can prove the theorem following Heckman and Honoré(1990).

Theorem 3.1. If $\left(J_{i} \in\{f, h\}, Y_{f i}\right.$ if $\left.J_{i}=f, X_{i}\right)$ are all observed and generated under model (3.1)-(3.4), then under assumptions 3.1-3.4, $g_{f}, g_{h}$, and $G$ are identified on a set $\mathcal{X}^{*}$ that has measure 1 .

\section{(Proof in Appendix)}

A key theme of this chapter is that the basic structure of identification in this model is similar to identification of more general selection models so we explain this result in much detail. The basic structure of the proof we present below is similar to Heckman and Honoré's proof of their Theorems 10 and 12. We modify the proof to allow for the case where $Y_{h i}$ is not observed.

The proof in the appendix is more precise, but in the text we present the basic ideas. We follow a structure analogous to the parametric empirical approach when the residuals are normally distributed as presented in section 3.1. First we consider identification of the occupational choice given only observable covariates and the choice model. This is the nonparametric analogue of the reduced form probit. Second we estimate $g_{f}$ given the data on $Y_{f i}$ which is the analogue of the second stage of the Heckman two step, and is more broadly the nonparametric version of the classical selection model. In the third step we consider the nonparametric analogue of identification of the structural probit. Since we will have already established identification of $g_{f}$, identification of this part of the model boils down to identification of $g_{h}$. Finally in the fourth step we consider identification of $G$ (the joint distribution of $\left.\left(\varepsilon_{f i}, \varepsilon_{h i}\right)\right)$. We discuss each of these steps in order.

To map the Roy model into our formal definition of identification presented in section 2.2, the model is determined by $\theta=\left(g_{f}, g_{h}, G, F_{x}\right)$ where $F_{x}$ is the joint distribution of $\left(X_{f i}, X_{h i}, X_{0 i}\right)$. The observable data here is $\left(X_{f i}, X_{h i}, X_{0 i}, J_{i}, 1\left(J_{i}=f\right) Y_{f i}\right)$. Thus $P$ 
is the joint distribution of this observable data and $\Theta(P)$ represents the possible data generating processes consistent with $P$.

\section{Step 1: Identification of Choice Model}

The nonparametric identification of this model is established in Matzkin (1992). We can write the model as

$$
\begin{aligned}
\operatorname{Pr}\left(J_{i}=f \mid X_{i}=x\right) & =\operatorname{Pr}\left(\varepsilon_{h i}-\varepsilon_{f i}<g_{f}\left(x_{f}, x_{0}\right)-g_{h}\left(x_{h}, x_{0}\right)\right) \\
& =G_{h-f}\left(g_{f}\left(x_{f}, x_{0}\right)-g_{h}\left(x_{h}, x_{0}\right)\right)
\end{aligned}
$$

where $G_{h-f}$ is the distribution function of $\varepsilon_{h i}-\varepsilon_{f i}$.

Using data only on choices, this model is only identified up to a monotonic transformation. To see why, note that we can write $J_{i}=f$ when

$$
g_{f}\left(x_{f}, x_{0}\right)-g_{h}\left(x_{h}, x_{0}\right)>\varepsilon_{h i}-\varepsilon_{f i}
$$

but this is equivalent to the condition

$$
M\left(g_{f}\left(x_{f}, x_{0}\right)-g_{h}\left(x_{h}, x_{0}\right)\right)>M\left(\varepsilon_{h i}-\varepsilon_{f i}\right)
$$

where $M($.$) is any strictly increasing function. Clearly the model in equation (3.8) can-$ not be distinguished from an alternative model in equation (3.9). This is the nonparametric analog of the problem that the scale (i.e., the variance of $\varepsilon_{h i}-\varepsilon_{f i}$ ) and location (only the difference between $g_{f}\left(x_{f}, x_{0}\right)$ and $g_{h}\left(x_{h}, x_{0}\right)$ but not the level of either) of the parametric binary choice model are not identified. Without loss of generality we can normalize the model up to a monotonic transformation. There are many ways to do this. A very convenient normalization is to choose the transformation $M(\cdot)=G_{h-f}(\cdot)$ because $G_{h-f}\left(\varepsilon_{h i}-\varepsilon_{f i}\right)$ has a uniform distribution. ${ }^{6}$ So we define

$$
\begin{aligned}
\varepsilon_{i} & \equiv G_{h-f}\left(\varepsilon_{h i}-\varepsilon_{f i}\right) \\
g(x) & \equiv G_{h-f}\left(g_{f}\left(x_{f}, x_{0}\right)-g_{h}\left(x_{h}, x_{0}\right)\right) .
\end{aligned}
$$

\footnotetext{
${ }^{6}$ To see why note that for any $x, \operatorname{Pr}\left(G_{h-f}\left(\varepsilon_{h i}-\varepsilon_{f i}\right)<x\right)=\operatorname{Pr}\left(\varepsilon_{h i}-\varepsilon_{f i} \leq G_{h-f}^{-1}(x)\right)=$ $\left.G_{h-f}\left(G_{h-f}^{-1}(x)\right)\right)=x$.
} 
Then

$$
\begin{aligned}
\operatorname{Pr}\left(J_{i}=f \mid X_{i}=x\right) & =\operatorname{Pr}\left(g_{f}\left(x_{f}, x_{0}\right)-g_{h}\left(x_{h}, x_{0}\right)>\varepsilon_{h i}-\varepsilon_{f i}\right) \\
& =\operatorname{Pr}\left(G_{h-f}\left(g_{f}\left(x_{f}, x_{0}\right)-g_{h}\left(x_{h}, x_{0}\right)\right)>G_{h-f}\left(\varepsilon_{h i}-\varepsilon_{f i}\right)\right) \\
& =\operatorname{Pr}\left(\varepsilon_{i}<g(x)\right) \\
& =g(x) .
\end{aligned}
$$

Thus we have established that we can (i) write the model as $J_{i}=f$ if and only if $g\left(X_{i}\right)>\varepsilon_{i}$ where $\varepsilon_{i}$ is uniform $[0,1]$ and (ii) that $g$ is identified.

This argument can be mapped into our formal definition of identification from Section 2.2 above. The goal here is identification of $g$ so we $\operatorname{define} \Psi(\theta)=g$. Note that even though $g$ is not part of $\theta$, it is a known function of the components of $\theta$. The key set now is $\Psi(\Theta(P))$ which is now defined as the set of possible values $g$ that could have generated the joint distribution of $\left(X_{f i}, X_{h i}, X_{0 i}, J_{i}, 1\left(J_{i}=f\right) Y_{f i}\right)$. Since $\operatorname{Pr}\left(J_{i}=f \mid X_{i}=x\right)=g(x)$, no other possible value of $g$ could generate the data. Thus $\Psi(\Theta(P))$ only contains the true value and is thus a singleton.

\section{Step 2: Identification of the Wage Equation $g_{f}$}

Next consider identification of $g_{f}$. Median regression identifies

$$
\operatorname{Med}\left(Y_{i} \mid X_{i}=x, J_{i}=f\right)=g_{f}\left(x_{f}, x_{0}\right)+\operatorname{Med}\left(\varepsilon_{f i} \mid X_{i}=x, \varepsilon_{i}<g(x)\right)
$$

The goal is to identify $g_{f}\left(x_{f}, x_{0}\right)$. The problem is that when we vary $\left(x_{f}, x_{0}\right)$ we also typically vary $\operatorname{Med}\left(\varepsilon_{f i} \mid X_{i}=x, g(x)>\varepsilon_{i}\right)$. This is the standard selection problem. Because we can add any constant to $g_{f}$ and subtract it from $\varepsilon_{f i}$ without changing the model, a normalization that allows us to pin down the location of $g_{f}$ is that $\operatorname{Med}\left(\varepsilon_{f i}\right)=0$. The problem is that this is the unconditional median rather than the conditional one. The solution here is what is often referred to as identification at infinity (e.g. Chamberlain, 1986, or Heckman, 1990). For some value $\left(x_{f}, x_{0}\right)$ suppose we can find a value of $x_{h}$ to send $\operatorname{Pr}\left(\varepsilon_{i}<g(x)\right)$ arbitrarily close to one. It is referred to as identification at infinity because if $g_{h}$ were linear in the exclusion restriction $x_{h}$ this could be achieved by sending $x_{h} \rightarrow-\infty$. In our fishing/hunting example, this could be sending the price of rabbits to zero which in turn sends log income from hunting to 
$-\infty$. Then notice that ${ }^{7}$

$$
\begin{aligned}
\lim _{g(x) \rightarrow 1} \operatorname{Med}\left(Y_{i} \mid X_{i}=x, J_{i}=f\right) & =g_{f}\left(x_{f}, x_{0}\right)+\lim _{g(x) \rightarrow 1} \operatorname{Med}\left(\varepsilon_{f i} \mid \varepsilon_{i} \leq g(x)\right)=g_{f}\left(x_{f}, x_{0}\right)+\operatorname{Med}\left(\varepsilon_{f i} \mid \varepsilon_{i} \leq 1\right) \\
& =g_{f}\left(x_{f}, x_{0}\right)+\operatorname{Med}\left(\varepsilon_{f i}\right) \\
& =g_{f}\left(x_{f}, x_{0}\right)
\end{aligned}
$$

Thus $g_{f}$ is identified.

Conditioning on $x$ so that $\operatorname{Pr}\left(J_{i}=1 \mid X_{i}=x\right)$ is arbitrarily close to one is essentially conditioning on a group of individuals for whom there is no selection, and thus there is no selection problem. Thus we are essentially saying that if we can condition on a group of people for whom there is no selection we can solve the selection bias problem.

While this may seem like cheating, without strong functional form assumptions it is necessary for identification. To see why, suppose there is some upper bound of $\operatorname{supp}\left[g\left(X_{i}\right)\right]$ equal to $g^{u}<1$ which would prevent us from using this type of argument. Consider any potential worker with a value of $\varepsilon_{i}>g^{u}$. For those individuals it must be the case that

$$
\varepsilon_{i}>g\left(X_{i}\right)
$$

so they must always be a hunter. As a result, the data is completely uninformative about the distribution of $\varepsilon_{f i}$ for these individuals. For this reason the unconditional median of $\varepsilon_{f i}$ would not be identified. We will discuss approaches to dealing with this potential problem in the Treatment Effect section below.

To relate this to the framework from Section 2.2 above now we define $\Psi(\theta)=g_{f}$, so $\Psi(\Theta(P))$ contains the values of $g_{f}$ consistent with $P$. However since

$$
\lim _{g(x) \rightarrow \infty} \operatorname{Med}\left(Y_{f} \mid X_{i}=x, J_{i}=f\right)=g_{f}\left(x_{f}, x_{0}\right)
$$

$g_{f}$ is the only element of $\Psi(\Theta(P))$, thus it is identified.

\section{Identification of the Slope Only without "Identification at Infinity"}

If one is only interested in identifying the "slope" of $g_{f}$ and not the intercept, one can avoid using an identification at infinity argument. That is, for any two points $\left(x_{f}, x_{0}\right)$ and $\left(\tilde{x}_{f}, \tilde{x}_{0}\right)$, consider identifying the difference $g_{f}\left(x_{f}, x_{0}\right)-g_{f}\left(\tilde{x}_{f}, \tilde{x}_{0}\right)$. The

\footnotetext{
${ }^{7}$ We are using loose notation here. What we mean by $\lim _{g(x) \rightarrow 1}$ is to hold $\left(x_{f}, x_{0}\right)$ fixed, but take a sequence of values of $x_{h}$ so that $g(x) \rightarrow 1$.
} 
key to identification is the existence of the exclusion restriction $X_{h i}$. For these two points, suppose we can find values $x_{h}$ and $\tilde{x}_{h}$ so that

$$
g\left(x_{f}, x_{h}, x_{0}\right)=g\left(\tilde{x}_{f}, \tilde{x}_{h}, \tilde{x}_{0}\right) .
$$

There may be many pairs of $\left(x_{h}, \tilde{x}_{h}\right)$ that satisfy this equality and we could choose any of them. Define $\tilde{x} \equiv\left(\tilde{x}_{f}, \tilde{x}_{h}, \tilde{x}_{0}\right)$. The key aspect of this is that since $g(x)=g(\tilde{x})$, and thus the probability of being a fisherman is the same given the two sets of points, then the bias terms are also the same: $\operatorname{Med}\left(\varepsilon_{f i} \mid \varepsilon_{i}<g(x)\right)=\operatorname{Med}\left(\varepsilon_{f i} \mid \varepsilon_{i}<g(\tilde{x})\right)$.

This allows us to write

$$
\begin{aligned}
\operatorname{Med}\left(Y_{i} \mid X_{i}=x, J_{i}=f\right) & -\operatorname{Med}\left(Y_{i} \mid X_{i}=\tilde{x}, J_{i}=f\right) \\
= & g_{f}\left(x_{f}, x_{0}\right)+\operatorname{Med}\left(\varepsilon_{f i} \mid \varepsilon_{i}<g(x)\right) \\
& -\left[g_{f}\left(\tilde{x}_{f}, \tilde{x}_{0}\right)+\operatorname{Med}\left(\varepsilon_{f i} \mid \varepsilon_{i}<g(\tilde{x})\right)\right] \\
= & g_{f}\left(x_{f}, x_{0}\right)-g_{f}\left(\tilde{x}_{f}, \tilde{x}_{0}\right) .
\end{aligned}
$$

As long as we have sufficient variation in $X_{h i}$ we can do this everywhere and identify $g_{f}$ up to location.

\section{Step 3: Identification of $g_{h}$}

In terms of identifying $g_{h}$, the exclusion restriction that influences wages as a fisherman but not as a hunter (i.e. $\left.X_{f i}\right)$ will be crucial. Consider identifying $g_{h}\left(x_{h}, x_{0}\right)$ for any particular value $\left(x_{h}, x_{0}\right)$. The key here is finding a value of $x_{f}$ so that

$$
\operatorname{Pr}\left(J_{i}=f \mid X_{i}=\left(x_{f}, x_{h}, x_{0}\right)\right)=0.5 .
$$

Assumption 3.2 guarantees that we can do this. To see why equation (3.10) is useful, note that it must be that for this value of $\left(x_{f}, x_{h}, x_{0}\right)$

$$
0.5=\operatorname{Pr}\left(\varepsilon_{h i}-\varepsilon_{f i} \leq g_{f}\left(x_{f}, x_{0}\right)-g_{h}\left(x_{h}, x_{0}\right)\right) .
$$

But the fact that $\varepsilon_{h i}-\varepsilon_{f i}$ has median zero implies that

$$
g_{h}\left(x_{h}, x_{0}\right)=g_{f}\left(x_{f}, x_{0}\right)
$$


Since $g_{f}$ is identified, $g_{h}$ is identified from this expression. ${ }^{8}$

Again to relate this to the framework in Section 2.2 above, now $\Psi(\theta)=g_{h}$ and $\Psi(\Theta(p))$ is the set of functions $g_{h}$ that are consistent with $P$. Above we showed that if $\operatorname{Pr}\left(J_{i}=f \mid X_{i}=x\right)=0.5$, then $g_{h}\left(x_{h}, x_{0}\right)=g_{f}\left(x_{f}, x_{0}\right)$. Thus since we already showed that $g_{f}$ is identified, $g_{h}$ is the only element of $\Psi(\Theta(p))$.

\section{Step 4: Identification of $G$}

Next consider identification of $G$ given $g_{f}$ and $g_{h}$. We will show how to identify the joint distribution of $\left(\varepsilon_{f i}, \varepsilon_{h i}\right)$ closely following the exposition of Heckman and Taber (2008). Note that from the data one can observe

$$
\begin{aligned}
& \operatorname{Pr}\left(J_{i}=f, Y_{f i}<s \mid X_{i}=x\right) \\
= & \operatorname{Pr}\left(g_{h}\left(x_{h}, x_{0}\right)+\varepsilon_{h i} \leq g_{f}\left(x_{f}, x_{0}\right)+\varepsilon_{f i}, g_{f}\left(x_{f}, x_{0}\right)+\varepsilon_{f i} \leq s\right) \\
= & \operatorname{Pr}\left(\varepsilon_{h i}-\varepsilon_{f i} \leq g_{f}\left(x_{f}, x_{0}\right)-g_{h}\left(x_{h}, x_{0}\right), \varepsilon_{f i} \leq s-g_{f}\left(x_{f}, x_{0}\right)\right)
\end{aligned}
$$

which is the cumulative distribution function of $\left(\varepsilon_{h i}-\varepsilon_{f i}, \varepsilon_{f i}\right)$ evaluated at the point $\left(g_{f}\left(x_{f}, x_{0}\right)-g_{h}\left(x_{h}, x_{0}\right), s-g_{f}\left(x_{f}, x_{0}\right)\right)$. By varying the point of evaluation one can identify the joint distribution of $\left(\varepsilon_{h i}-\varepsilon_{f i}, \varepsilon_{f i}\right)$ from which one can derive the joint distribution of $\left(\varepsilon_{f i}, \varepsilon_{h i}\right)$.

Finally in terms of the identification conditions in Section 2.2 above, now $\Psi(\theta)=G$ and $\Psi(\Theta(P))$ is the set of distributions $G$ consistent with $P$. Since $G$ is uniquely defined by the expression (3.12) and since everything else in this expression is identified, $G$ is the only element of $\Psi(\Theta(P))$.

\subsection{Relaxing Independence between Observables and Unob- servables.}

For expositional purposes we focus on the case in which the observables are independent of the unobservables, but relaxing these assumptions is easy to do. The simplest case is to allow for a general relationship between $X_{0 i}$ and $\left(\varepsilon_{f i}, \varepsilon_{h i}\right)$. To see how easy this

\footnotetext{
${ }^{8}$ Note that Heckman and Honoré (1990) choose a different normalization. Rather than normalizing the median of $\varepsilon_{h i}-\varepsilon_{f i}$ to zero (which is convenient in the case in which $Y_{h i}$ is not observed) they normalize the median of $\varepsilon_{h i}$ to zero (which is more convenient in their case). Since this is just a normalization, it is innocuous. After identifying the model under our normalization we could go back to redefine the model in terms of theirs.
} 
is, consider a case in which $X_{0 i}$ is just binary, for example denoting men and women. Independence seems like a very strong assumption in this case. For example, the distribution of unobserved preferences might be different for women and men, leading to different selection patterns. In order to allow for this, we could identify and estimate the Roy model separately for men and for women. Expanding from binary $X_{0 i}$ to finite support $X_{0 i}$ is trivial, and going beyond that to continuous $X_{0 i}$ is straightforward. Thus one can relax the independence assumption easily. But for expositional purposes we prefer our specification.

The distinction between $X_{f i}$ and $X_{0 i}$ was not important in steps 1 and 2 of our discussion above. When one is only interested in the outcome equation $Y_{f i}=g_{f}\left(X_{f i}, X_{0 i}\right)+$ $\varepsilon_{f i}$, relaxing the independence assumption between $X_{f i}$ and $\left(\varepsilon_{f i}, \varepsilon_{h i}\right)$ can be done as well. However, in step 3 this distinction is important in identifying $g_{h}$ and the independence assumption is not easy to relax.

If we allow for general dependence between $X_{0 i}$ and $\left(\varepsilon_{f i}, \varepsilon_{h i}\right)$, the "identification at infinity" argument becomes more important as the argument about "Identification of the Slope Only without Identification at Infinity" no longer goes through. In that case the crucial feature of the model was that $\operatorname{Med}\left(\varepsilon_{f i} \mid \varepsilon_{i}<g(x)\right)=\operatorname{Med}\left(\varepsilon_{f i} \mid \varepsilon_{i}<g(\tilde{x})\right)$. However, without independence this is no longer generally true because $\operatorname{Med}\left(\varepsilon_{f i} \mid X_{i}=\right.$ $\left.x, J_{i}=f\right)=\operatorname{Med}\left(\varepsilon_{f i} \mid X_{0 i}=x_{0}, \varepsilon_{i}<g(x)\right)$. Thus even if $g(x)=g(\tilde{x})$, when $x_{0} \neq \tilde{x}_{0}$, in general $\operatorname{Med}\left(\varepsilon_{f i} \mid X_{0 i}=x_{0}, \varepsilon_{i}<g(x)\right) \neq \operatorname{Med}\left(\varepsilon_{f i} \mid X_{0 i}=\tilde{x}_{0}, \varepsilon_{i}<g(\tilde{x})\right)$.

\subsection{The Importance of Exclusion Restrictions}

We now show that the model is not identified in general without an exclusion restriction. ${ }^{9}$ Consider a simplified version of the model,

$$
\begin{gathered}
J_{i}= \begin{cases}f & \text { if } g\left(X_{i}\right)-\varepsilon_{i} \geq 0 \\
h & \text { otherwise }\end{cases} \\
Y_{f i}=g_{f}\left(X_{i}\right)+\varepsilon_{f i}
\end{gathered}
$$

\footnotetext{
${ }^{9}$ An exception is Buera (2006), who allows for general functional forms and does not need an exclusion restriction. Assuming wages are observed in both sectors, and making stronger use of the independence assumption between the observables and the unobservables, he shows that the model can be identified without exclusion restrictions.
} 
where $\varepsilon_{i}$ is uniform $(0,1)$ and $\left(\varepsilon_{i}, \varepsilon_{f i}\right)$ is independent of $X_{i}$ with distribution $G$ and we use the location normalization $\operatorname{Med}\left(\varepsilon_{f i} \mid X_{i}\right)=0$. As in Section 3.2, we observe $X_{i}$, whether $J_{i}=f$ or $h$, and if $J_{i}=f$ then we observe $Y_{f i}$.

We can think about estimating the model from the median regression

$$
\begin{aligned}
\operatorname{Med}\left[Y_{f i} \mid X_{i}=x\right] & =g_{f}\left(X_{i}\right)+\operatorname{Med}\left[\varepsilon_{f i} \mid X_{i}=x\right] \\
& =g_{f}\left(X_{i}\right)+\operatorname{Med}\left[\varepsilon_{f i} \mid g\left(X_{i}\right)>\varepsilon_{i}\right] \\
& =g_{f}\left(X_{i}\right)+h\left(g\left(X_{i}\right)\right)
\end{aligned}
$$

Under the assumption that $\operatorname{Med}\left(\varepsilon_{f i} \mid X_{i}\right)=0$ it must be the case that $h(1)=0$, but this is our only restriction on $h$ and $g$. Thus the model above has the same conditional median as an alternative model

$$
\operatorname{Med}\left[Y_{f i} \mid X_{i}=x\right]=\widetilde{g}_{f}\left(X_{i}\right)+\widetilde{h}\left(g\left(X_{i}\right)\right)
$$

where $\widetilde{g}_{f}\left(X_{i}\right)=g_{f}\left(X_{i}\right)+k\left(g\left(X_{i}\right)\right)$ and $\widetilde{h}\left(g\left(X_{i}\right)\right)=h\left(g\left(X_{i}\right)\right)-k\left(g\left(X_{i}\right)\right)$. Equations (3.13) and (3.14) are observationally equivalent. Without an exclusion restriction, it is impossible to tell if observed income from working varies with $X_{i}$ because it varies with $g_{f}$ or because it varies with the labor force participation rate and thus the extent of selection. Thus the models in equations (3.13) and (3.14) are not distinguishable using conditional medians.

To show the two models are indistinguishable using using the full joint distribution of the data, consider an alternative data generating model with the same first stage, but now $Y_{f i}$ is determined by

$$
Y_{f i}=\widetilde{g}_{f}\left(X_{i}\right)+\widetilde{\varepsilon}_{f i}
$$

where $\widetilde{\varepsilon}_{f i}$ is independent of $X_{i}$ with $\operatorname{Med}\left(\widetilde{\varepsilon}_{f i} \mid X_{i}\right)=0$. Let $\widetilde{G}\left(\varepsilon_{i}, \widetilde{\varepsilon}_{f i}\right)$ be the joint distribution of $\left(\varepsilon_{i}, \widetilde{\varepsilon}_{f i}\right)$ in the alternative model. We will continue to assume that in the alternative model $\widetilde{g}_{f}\left(X_{i}\right)=g_{f}\left(X_{i}\right)+k\left(g\left(X_{i}\right)\right)$. The question is whether the alternative model to be able to generate the same data distribution.

In the true model

$$
\begin{aligned}
\operatorname{Pr}\left(\varepsilon_{i} \leq g(x), Y_{f i}<y\right) & =\operatorname{Pr}\left(\varepsilon_{i} \leq g(x), g_{f}(x)+\varepsilon_{f i} \leq y\right) \\
& =G\left(g(x), y-g_{f}(x)\right)
\end{aligned}
$$


In the alternative model

$$
\begin{aligned}
\operatorname{Pr}\left(\varepsilon_{i} \leq g(x), Y_{f i}<y\right) & =\operatorname{Pr}\left(\varepsilon_{i} \leq g(x), \widetilde{g}_{f}(x)+\widetilde{\varepsilon}_{f i} \leq y\right) \\
& =\widetilde{G}\left(g(x), y-\widetilde{g}_{f}(x)\right)
\end{aligned}
$$

Thus these two models generate exactly the same joint distribution of data and cannot be separately identified as long as we define $\widetilde{G}$ so that ${ }^{10}$

$$
\begin{aligned}
\widetilde{G}\left(g(x), y-\widetilde{g}_{f}(x)\right) & =G\left(g(x), y-g_{f}(x)\right) \\
& =G\left(g(x), y-\widetilde{g}_{f}(x)+k(g(x))\right) .
\end{aligned}
$$

\section{The Generalized Roy Model}

We next consider the "Generalized Roy Model" (as defined in e.g. Heckman and Vytlacil, 2007a). The basic Roy model assumes that workers only care about their income. The Generalized Roy Model allows workers to care about non-pecuniary aspects of the job as well. Let $U_{f i}$ and $U_{h i}$ be the utility that individual $i$ would receive from being a fisherman or a hunter respectively where for $j \in\{f, h\}$,

$$
U_{j i}=Y_{j i}+\varphi_{j}\left(Z_{i}, X_{0 i}\right)+\nu_{j i}
$$

where $\varphi_{j}\left(Z_{i}, X_{0 i}\right)$ represents the non-pecuniary utility gain from observables $Z_{i}$ and $X_{0 i}$. The variable $Z_{i}$ allows for the fact that there may be other variables that affect the taste for hunting versus fishing directly, but do not affect wages in either sector. ${ }^{11}$ Note that we are imposing separability between $Y_{j i}$ and $\varphi_{j}$. In general we can provide conditions in which the results presented here will go through if we relax this assumption but we impose it for expositional simplicity. The occupation is now defined as

$$
J_{i}=\left\{\begin{array}{ll}
f & \text { if } U_{f i}>U_{h i} \\
h & \text { if } U_{f i} \leq U_{h i}
\end{array} .\right.
$$

\footnotetext{
${ }^{10}$ One cannot do this with complete freedom as one needs $\widetilde{G}$ to be a legitimate c.d.f. That is, it must be nondecreasing in both of its arguments. However, there will typically be many examples of $k$ for which $\widetilde{G}$ is a cdf and the model is not identified. For example, if $k$ is a nondecreasing function $\widetilde{G}$ will be a legitimate c.d.f.

${ }^{11}$ In principle some of the elements of $Z_{i}$ may affect $\varphi_{f}$ and others may affect $\varphi_{h}$, but this distinction will not be important here so we use the most general notation.
} 
We continue to assume that

$$
\begin{gathered}
Y_{f i}=g_{f}\left(X_{f i}, X_{0 i}\right)+\varepsilon_{f i} \\
Y_{h i}=g_{h}\left(X_{h i}, X_{0 i}\right)+\varepsilon_{h i} \\
Y_{i}=\left\{\begin{array}{ll}
Y_{f i} & \text { if } J_{i}=f \\
Y_{h i} & \text { if } J_{i}=h
\end{array} .\right.
\end{gathered}
$$

It will be useful to define a reduced form version of this model. Note that people fish when

$$
\begin{aligned}
0 & <U_{f i}-U_{h i} \\
& =\left(Y_{f i}+\varphi_{f}\left(Z_{i}, X_{0 i}\right)+\nu_{f i}\right)-\left(Y_{h i}+\varphi_{h}\left(Z_{i}, X_{0 i}\right)+\nu_{h i}\right) \\
& =g_{f}\left(X_{f i}, X_{0 i}\right)+\varphi_{f}\left(Z_{i}, X_{0 i}\right)-g_{h}\left(X_{h i}, X_{0 i}\right)-\varphi_{h}\left(Z_{i}, X_{0 i}\right)+\varepsilon_{f i}+\nu_{f i}-\varepsilon_{h i}-\nu_{h i} .
\end{aligned}
$$

In the previous section we described how the choice model can only be identified up to a monotonic transform and that assuming the error term is uniform is a convenient

normalization. We do the same thing here. Let $F^{*}$ be the distribution function of $\varepsilon_{h i}+\nu_{h i}-\varepsilon_{f i}-\nu_{f i}$. Then we define

$$
\begin{aligned}
\nu_{i} & \equiv F^{*}\left(\varepsilon_{h i}+\nu_{h i}-\varepsilon_{f i}-\nu_{f i}\right) \\
\varphi\left(Z_{i}, X_{i}\right) & \equiv F^{*}\left(g_{f}\left(X_{f i}, X_{0 i}\right)+\varphi_{f}\left(Z_{i}, X_{0 i}\right)-g_{h}\left(X_{h i}, X_{0 i}\right)-\varphi_{h}\left(Z_{i}, X_{0 i}\right)\right) .
\end{aligned}
$$

As above, this normalization is convenient because it is straightforward to show that

$$
J_{i}=f \text { when } \varphi\left(Z_{i}, X_{i}\right)>\nu_{i}
$$

and that $\nu_{i}$ is uniformly distributed on the unit interval.

We assume that the econometrician can observe the occupations of the worker and the wages that they receive in their chosen occupations as well as $\left(X_{i}, Z_{i}\right)$.

\subsection{Identification}

It turns out that the basic assumptions that allow us to identify the Roy model also allow us to identify the generalized Roy model.

We start with the reduced form model in which we need two more assumptions. 
Assumption 4.1. $\left(\nu_{i}, \varepsilon_{f i}, \varepsilon_{h i}\right)$ is continuously distributed and is independent of $\left(Z_{i}, X_{i}\right)$. Furthermore, $\nu_{i}$ is distributed uniform on the unit interval and the medians of both $\varepsilon_{f i}$ and $\varepsilon_{\text {hi }}$ are zero.

Assumption 4.2. The support of $\varphi\left(Z_{i}, x\right)$ is $[0,1]$ for all $x \in \operatorname{supp}\left(X_{i}\right)$.

We also slightly extend the restrictions on the functions to include $\varphi_{f}$ and $\varphi_{h}$.

Assumption 4.3. $\left(Z_{i}, X_{i}\right)=\left(Z_{i}, X_{f i}, X_{h i}, X_{0 i}\right)$ can be written as $\left(Z_{i}^{c}, Z_{i}^{d}, X_{f i}^{c}, X_{f i}^{d}, X_{h i}^{c}, X_{h i}^{d}, X_{0 i}^{c}, X_{0 i}^{d}\right)$ where the elements of $\left(Z_{i}^{c}, X_{f i}^{c}, X_{h i}^{c}, X_{0 i}^{c}\right)$ are continuously distributed (no point has positive mass), and $\left(Z_{i}^{d}, X_{f i}^{d}, X_{h i}^{d}, X_{0 i}^{d}\right)$ is distributed discretely (all support points have positive mass).

Assumption 4.4. For any $\left(z^{d}, x_{f}^{d}, x_{h}^{d}, x_{0}^{d}\right) \in \operatorname{supp}\left(Z_{i}^{d}, X_{f i}^{d}, X_{h i}^{d}, X_{0 i}^{d}\right), g_{f}\left(x_{f}^{c}, x_{f}^{d}, x_{0}^{c}, x_{0}^{d}\right)$, $g_{h}\left(x_{h}^{c}, x_{h}^{d}, x_{0}^{c}, x_{0}^{d}\right), \varphi_{f}\left(z^{c}, z^{d}, x_{0}^{c}, x_{0}^{d}\right)$ and $\varphi_{h}\left(z^{c}, z^{d}, x_{0}^{c}, x_{0}^{d}\right)$ are almost surely continuous across

$$
\left(z^{c}, x^{c}\right) \in \operatorname{supp}\left(Z_{i}^{c}, X_{i}^{c} \mid\left(Z_{i}^{d}, X_{i}^{d}\right)=\left(z^{d}, x^{d}\right)\right)
$$

Theorem 4.1. Under assumptions 4.1-4.4, $\varphi, g_{f}, g_{h}$ and the joint distribution of $\left(\nu_{i}, \varepsilon_{f i}\right)$ and of $\left(\nu_{i}, \varepsilon_{h i}\right)$ are identified from the joint distribution of $\left(J_{i}, Y_{i}\right)$ on a set $\mathcal{X}^{*}$ that has measure 1 where $\left(J_{i}, Y_{i}\right)$ are generated by model (4.1)-(4.4).

\section{(Proof in Appendix)}

The intuition for identification follows directly from the intuition given for the basic Roy model. We show this in 3 steps:

1. Identification of $\varphi$ is like the "Step 1: identification of choice model" section. We can only identify $\varphi$ up to a monotonic transformation for exactly the same reason given in that section. We impose the normalization that $\nu_{i}$ is uniform in assumption 4.2. Given that assumption

$$
\operatorname{Pr}\left(J_{i}=f \mid Z_{i}=z, X_{i}=x\right)=\varphi(z, x)
$$

so identification of $\varphi$ from $\operatorname{Pr}\left(J_{i}=f \mid Z_{i}=z, X_{i}=x\right)$ comes directly. 
2. Identification of $g_{f}$ and $g_{h}$ are completely analogous to "Step 2: identification of $g_{f}$ " in section 3.2. That is

$$
\begin{aligned}
\lim _{\varphi(z, x) \rightarrow 1} & \operatorname{Med}\left(Y_{i} \mid Z_{i}=z, X_{i}=x, J_{i}=f\right) \\
& =g_{f}\left(x_{f}, x_{0}\right)+\lim _{\varphi(z, x) \rightarrow 1} \operatorname{Med}\left(\varepsilon_{f i} \mid Z_{i}=z, X_{i}=x, J_{i}=f\right) \\
& =g_{f}\left(x_{f}, x_{0}\right)+\lim _{\varphi(z, x) \rightarrow 1} \operatorname{Med}\left(\varepsilon_{f i} \mid \nu_{i} \leq \varphi(z, x)\right) \\
& =g_{f}\left(x_{f}, x_{0}\right)+\operatorname{Med}\left(\varepsilon_{f i}\right) \\
& =g_{f}\left(x_{f}, x_{0}\right) .
\end{aligned}
$$

The analogous argument works for $g_{h}$ when we send $\varphi(z, x) \rightarrow 0$.

3. Identification of the joint distribution of $\left(\nu_{i}, \varepsilon_{f i}\right)$ and of $\left(\nu_{i}, \varepsilon_{h i}\right)$ are analogous to the "Step 4: identification of $G$ " discussion in the Roy model. That is if we let $G_{\nu, \varepsilon_{f}}$ represent the joint distribution of $\left(\nu_{i}, \varepsilon_{f i}\right)$ then

$$
\begin{aligned}
\operatorname{Pr}\left(J_{i}=f, Y_{f i} \leq y \mid\left(Z_{i}, X_{i}\right)=(z, x)\right) & =\operatorname{Pr}\left(\nu_{i} \leq \varphi(z, x), g_{f}\left(x_{f}, x_{0}\right)+\varepsilon_{f i} \leq y\right) \\
& =G_{\nu, \varepsilon_{f}}\left(\varphi(z, x), y-g_{f}\left(x_{f}, x_{0}\right)\right) .
\end{aligned}
$$

The analogous argument works for the joint distribution of $\left(\nu_{i}, \varepsilon_{h i}\right)$.

Note that not all parameters are identified such as the non-pecuniary gain from fishing $\varphi_{f}-\varphi_{h}$. To identify the "structural" generalized Roy model we make two additional assumptions:

Assumption 4.5. The median of $\varepsilon_{h i}+\nu_{h i}-\varepsilon_{f i}-\nu_{f i}$ is zero.

Assumption 4.6. For any value of $\left(z, x_{0}\right) \in \operatorname{supp}\left(Z_{i}, X_{0 i}\right), g_{f}\left(X_{f i}, x_{0}\right)-g_{h}\left(X_{h i}, x_{0}\right)$ has full support (i.e. the whole real line).

Theorem 4.2. Under Assumptions 4.1-4.6, $\varphi_{f}-\varphi_{h}$, the distribution of $\left(\varepsilon_{h i}+\nu_{h i}-\varepsilon_{f i}-\nu_{f i}, \varepsilon_{f i}\right)$, and the distribution of $\left(\varepsilon_{h i}+\nu_{h i}-\varepsilon_{f i}-\nu_{f i}, \varepsilon_{h i}\right)$ are identified.

\section{(Proof in Appendix)}

Note that Theorem 4.1 gives the joint distribution of $\left(\nu_{i}, \varepsilon_{f i}\right)$ while Theorem 4.2 gives the joint distribution of $\left(\varepsilon_{h i}+\nu_{h i}-\varepsilon_{f i}-\nu_{f i}, \varepsilon_{f i}\right)$. Since $\nu_{i}=F^{*}\left(\varepsilon_{h i}+\nu_{h i}-\varepsilon_{f i}-\nu_{f i}\right)$, this really just amounts to saying that $F^{*}$ is identified. 
Furthermore, whereas $g_{f}$ and $g_{h}$ are identified in Theorem 4.1, $\varphi_{f}-\varphi_{h}$ is identified in Theorem 4.2. Recall $\varphi_{f}-\varphi_{h}$ is the added utility (measured in money) of being a fisherman relative to a hunter. The exclusion restrictions $X_{f i}$ and $X_{h i}$ help us identify this. These exclusion restrictions allow us to vary the pecuniary gains of the two sectors, holding preferences $\varphi_{f}-\varphi_{h}$ constant. Identification is analogous to the "Step 3: identification of $g_{h}$ " in the standard Roy model. To see where identification comes from, for every $\left(z, x_{0}\right)$ think about the following conditional median

$$
\begin{aligned}
0.5 & =\operatorname{Pr}\left(J_{i}=f \mid Z_{i}=z, X_{i}=x\right) \\
& =\operatorname{Pr}\left(\varepsilon_{h i}+\nu_{h i}-\varepsilon_{f i}-\nu_{f i} \leq g_{f}\left(x_{f}, x_{0}\right)+\varphi_{f}\left(z, x_{0}\right)-g_{h}\left(x_{h}, x_{0}\right)-\varphi_{h}\left(z, x_{0}\right)\right) .
\end{aligned}
$$

Since the median of $\varepsilon_{h i}+\nu_{h i}-\varepsilon_{f i}-\nu_{f i}$ is zero, this means that

$$
g_{f}\left(x_{f}, x_{0}\right)+\varphi_{f}\left(z, x_{0}\right)-g_{h}\left(x_{h}, x_{0}\right)-\varphi_{h}\left(z, x_{0}\right)=0,
$$

and thus

$$
\varphi_{f}\left(z, x_{0}\right)-\varphi_{h}\left(z, x_{0}\right)=g_{h}\left(x_{h}, x_{0}\right)-g_{f}\left(x_{f}, x_{0}\right) .
$$

Because $g_{f}$ and $g_{h}$ is identified, $\varphi_{f}-\varphi_{h}$ is identified also. The argument above shows that we do not need both $X_{f i}$ and $X_{h i}$, we only need $X_{f i}$ or $X_{h i}$.

Suppose there is no variable that affects earnings in one sector but not preferences $\left(X_{f i}\right.$ or $\left.X_{h i}\right)$. An alternative way to identify $\varphi_{f}-\varphi_{h}$ is to use a cost measured in dollars. Consider the linear version of the model with normal errors and without exclusion restrictions $\left(X_{h i}, X_{f i}\right)$ so that

$$
\begin{aligned}
g_{h}\left(x_{0}\right) & =x_{0 i}^{\prime} \gamma_{h} \\
g_{f}\left(x_{0}\right) & =x_{0 i}^{\prime} \gamma_{f} \\
\varphi_{f}\left(z, x_{0}\right)-\varphi_{h}\left(z, x_{0}\right) & =x_{0}^{\prime} \beta_{0}+z^{\prime} \beta_{z} .
\end{aligned}
$$

The reduced form probit is:

$$
\operatorname{Pr}\left(J_{i}=f \mid Z_{i}=z, X_{i}=x\right)=\Phi\left(x_{0 i}^{\prime} \frac{\gamma_{f}-\gamma_{h}+\beta_{0}}{\sigma}+z_{i}^{\prime} \frac{\beta_{z}}{\sigma}\right)
$$

where $\sigma$ is the standard deviation of $\varepsilon_{h i}+\nu_{h i}-\varepsilon_{f i}-\nu_{f i}$. Theorem 4.1 above establishes that the functions $g_{f}$ and $g_{h}$ (i.e., $\gamma_{f}$ and $\gamma_{h}$ ) as well as the variance of $\varepsilon_{h i}$ and $\varepsilon_{f i}$ are identified. We still need to identify $\beta_{0}, \beta_{z}$ and $\sigma$. Thus we are able to identify

$$
\frac{\gamma_{f}-\gamma_{h}+\beta_{0}}{\sigma} \text { and } \frac{\beta_{z}}{\sigma} \text {. }
$$


If $\beta_{0}$ and $\beta_{z}$ are scalars we still have three parameters $\left(\beta_{0}, \beta_{z}, \sigma\right)$ and two restrictions $\left(\frac{\gamma_{f}-\gamma_{h}+\beta_{0}}{\sigma}, \frac{\beta_{z}}{\sigma}\right)$. If they are not scalars, we still have one more parameter than restriction. However suppose that one of the exclusion restrictions represents a cost variable that is measured in the same units as $Y_{f i}-Y_{h i}$. For example in a schooling case suppose that $Y_{f i}$ represents the present value of earnings as a college graduate, $Y_{h i}$ represents the present value of high school graduate as a college graduate, and the exclusion restriction, $Z_{i}$, represents the present value of college tuition. In this case $\beta_{z}=-1$ the coefficient on $Z_{i}$ is $-1 / \sigma$, so $\sigma$ is identified. Given $\sigma$ it is very easy to show that the rest of the parameters are identified as well. Heckman, Lochner, and Taber (1998) provide an example of this argument using tuition as in the style above. In section 7.3 we discuss Heckman and Navarro (2007) who use this approach as well.

\subsection{Lack of Identification of the Joint Distribution of $\left(\varepsilon_{f i}, \varepsilon_{h i}\right)$}

In pointing out what is identified in the model it is also important to point out what is not identified. Most importantly in the generalized Roy model we were able to identify the joint distribution between the error terms in the selection equation and each of the outcomes, but not the joint distribution of the variables in the outcome equation. In particular the joint distribution between the error terms $\left(\varepsilon_{f i}, \varepsilon_{h i}\right)$ is not identified. Even strong functional form assumptions will not solve this problem. Fir example, it is easy to show that in the joint normal model the covariance of $\left(\varepsilon_{f i}, \varepsilon_{h i}\right)$ is not identified.

\subsection{Are Functional Forms Innocuous? Evidence from Catholic Schools}

As the theorems above make clear, nonparametric identification requires exclusion restrictions. However, completely parametric models typically do not require exclusion restrictions. In specific empirical examples, identification could primarily be coming from the exclusion restriction or identification could be coming primarily from the functional form assumptions (or some combination between the two). When researchers use exclusion restrictions in data, it is important to be careful about which assumptions are important.

We describe one example from Altonji, Elder, and Taber (2005b). Based on Evans and Schwab (1995), Neal (1997), and Neal and Grogger (2000) they consider a bivariate 
probit model of Catholic schooling and college attendance.

$$
\begin{aligned}
C H_{i} & =1\left(X_{i}^{\prime} \beta+\lambda Z_{i}+u_{i}>0\right) \\
Y_{i} & =1\left(\alpha C H_{i}+X_{i}^{\prime} \gamma+\varepsilon_{i}>0\right),
\end{aligned}
$$

where $1(\cdot)$ is the indicator function taking the value one if its argument is true and zero otherwise, $\mathrm{CH}_{i}$ is a dummy variable indicating attendance at a Catholic school, and $Y_{i}$ is a dummy variable indicating college attendance. Identification of the effect of Catholic schooling on college attendance (or high school graduation) is the primary focus of these studies. The question at hand is in practice whether the assumed functional forms for $u_{i}$ and $\varepsilon_{i}$ are important for identifying the $\alpha$ coefficient and thus the effect of Catholic schools on college attendance.

The model in equations (4.7)-(4.8) is a minor extension of the generalized Roy model. The first key difference is that the outcome variable in equation (4.8) is binary (attend college or not), whereas in the case of the Generalized Roy model the outcomes were continuous (earnings in either sector). The second key difference is that the outcome equation for Catholic versus Non-Catholic school only differs in the intercept $(\alpha)$. The error term $\left(\varepsilon_{i}\right)$ and the slope coefficients $(\gamma)$ are restricted to be the same. Nevertheless, the machinery to prove non-parametric identification of the Generalized Roy model can be applied to this framework. ${ }^{12}$

Using data from the National Longitudinal Survey of 1972, Altonji, Elder, and Taber (2005b) consider an array of instruments and different specifications for equations (4.7) and (4.8) . In Table 1 we present a subset of their results. We show four different models. The "Single Equation Model" gives results in which selection into Catholic school is not accounted for. The first column gives results from a probit model (with point estimates, standard errors, and marginal effects). The second column give results from a Linear Probability model. Next we present the estimates of $\alpha$ from a Bivariate Probit models with alternative exclusion restrictions. The final row presents the results with no exclusion restrictions. Finally we also present results from an instrumental variable linear probability model with the same set of exclusion restrictions.

\footnotetext{
${ }^{12}$ Following Matzkin (1992), we need a monotonic normalization on the outcome model (such as assuming the error term is uniform). Once we have done this, proving identification of this model is almost identical to the generalized Roy model and is easily done with an exclusion restriction with sufficient support.
} 
Table 1

\begin{tabular}{|c|c|c|}
\hline \multicolumn{3}{|c|}{$\begin{array}{c}\text { Estimated Effects of Catholic Schools on College Attendance: } \\
\text { From Linear and Nonlinear Specifications }\end{array}$} \\
\hline \multicolumn{3}{|c|}{ Single Equation Models } \\
\hline & Probit & OLS \\
\hline & 0.239 & 0.239 \\
\hline \multicolumn{3}{|c|}{$[0.640]$} \\
\hline & $(0.198)$ & $(0.070)$ \\
\hline \multicolumn{3}{|c|}{ Two Equation Models } \\
\hline Excluded Variable & Bivariate Probit & 2SLS \\
\hline \multirow[t]{3}{*}{ Catholic } & 0.285 & -0.093 \\
\hline & {$[0.761]$} & \\
\hline & $(0.543)$ & $(0.324)$ \\
\hline \multirow[t]{3}{*}{ Catholic $\times$ Distance } & 0.478 & 2.572 \\
\hline & {$[1.333]$} & \\
\hline & $(0.516)$ & $(2.442)$ \\
\hline \multirow[t]{3}{*}{ None } & 0.446 & \\
\hline & {$[1.224]$} & \\
\hline & $(0.542)$ & \\
\hline
\end{tabular}

Notes: Source: Altonji, Elder, Taber (2005b). Urban Non-Whites from NLS-72.

The first set of results come from simple probits and from OLS.

The further results come from Bivariate Probits and from two stage least squares.

We present the marginal effect of Catholic high school attendance on college attendance.

[Point Estimate from Probit in Brackets]

(Standard Errors in Parentheses)

One can see that the marginal effect from the single equation probit is very similar to the OLS estimate. It indicates that college attendance rates are approximately 23.9 percentage points higher for Catholic high school graduates than for public high school 
graduates. The rest of the table presents results from three bivariate probit models and two instrumental variables models using alternative exclusion restrictions. The problem is clearest when the interaction between the student coming from a Catholic school and distance to the nearest Catholic school is used as an instrument. The 2SLS gives nonsensical results: a coefficient of 2.572 with an enormous standard error. This indicates that the instrument has little power. However, the bivariate probit result is more reasonable. It suggests that the true marginal causal effect is around 0.478 and the point estimate is statistically significant. This seems inconsistent with the 2SLS results which indicated that this exclusion restriction had very little power. However it is clear what is going on when we compare this result to the model at the bottom of the table without an exclusion restriction. The estimate is very similar with a similar standard error. The linearity and normality assumptions drive the results.

The case in which Catholic religion by itself is used as an instrument is less problematic. The IV result suggests a strong amount of positive selection but still yields a large standard error. The bivariate probit model suggests a marginal effect that is a bit larger than the OLS effect. However, note that the standard error for the model with and without an exclusion restriction are quite similar which seems inconsistent with the idea that the exclusion restriction is providing a lot of identifying information. Further note that the IV result suggests a strong positive selection bias while the bivariate probit without exclusion restrictions suggests a strong negative bias. The bivariate probit in which Catholic is excluded is somewhere between the two. This suggests that both functional form and exclusion restrictions are important in this case. We should emphasize the "suggests" part of this sentence as none of this is a formal test. It does, however, make one wonder how much trust to put in the bivariate probit results by themselves.

Another paper documenting the importance of functional form assumptions is Das et al. (2003), who estimate the return to education for young Australian women. They estimate equations for years of education, the probability of working, and wages. When estimating the wage equation they address both the endogeneity of years of education and also selection caused because we only observe wages for workers. They allow for flexibility in the returns to education (where the return depends on years of education) and also in the distribution of the residuals. They find that when they assume normality 
of the error terms, the return to education is approximately $12 \%$, regardless of years of education. However, once they allow for more flexible functional forms for the error terms, they find that the returns to education decline sharply with years of education. For example, they find that at 10 years of education, the return to education is over $15 \%$. However, at 14 years, the return to education is only about $5 \%$.

\section{$5 \quad$ Treatment Effects}

There is a very large literature on the estimation of treatment effects. For more complete summaries see Heckman and Robb (1986), Heckman, Lalonde and Smith (1999), Heckman and Vytlacil (2007a,2007b), Abbring and Heckman (2007), or Imbens and Wooldridge (2009). ${ }^{13}$ DiNardo and Lee (2010) provide a discussion that is complementary to ours. Our goal in this section is not to survey the whole literature but provide a brief summary and to put it into the context of identification of the Generalized Roy Model.

The goal of this literature is to estimate the value of receiving a treatment defined as:

$$
\pi_{i}=Y_{f i}-Y_{h i}
$$

In the context of the Roy model, $\pi_{i}$ is the income gain from moving from hunting to fishing. This income gain potentially varies across individuals in the population. Thus for people who choose to be fishermen, $\pi_{i}$ is positive and for people who choose to be hunters, $\pi_{i}$ is negative.

Estimation of treatment effects is of great interest in many literatures. The term "treatment effect" makes the most sense in the context the medical literature. Choice $f$ could represent taking a medical treatment (such as an experimental drug) while $h$ could represent no treatment. In that case $Y_{f i}$ and $Y_{h i}$ would represent some measure of health status for individual $i$ with and without the treatment. Thus the treatment effect $\pi_{i}$ is the effect of the drug on the health outcome for individual $i$.

The classic example in labor economics is job training. In that case, $Y_{f i}$ would

\footnotetext{
${ }^{13}$ There is also a substantial literature on the tradeoffs between different empirical approaches. Key papers include Leamer (1983), Heckman (1979, 1999, 2000), Angrist and Imbens (1999), Rosenzweig and Wolpin (2000), Deaton (2009), Heckman and Urzua (2009), Imbens (2009), Angrist and Pischke (2010), Sims (2010).
} 
represent a labor market outcome for individuals who received training and $Y_{h i}$ would represents the outcome in the absence of training.

In both the case of drug treatment and job training, empirical researchers have exploited randomized trials. Medical patients are often randomly assigned either a treatment or a placebo (i.e., a sugar pill that should have no effect on health). Likewise, many job training programs are randomly assigned. For example, in the case of the Job Training Partnership Act, a large number of unemployed individuals applied for job training (see e.g. Bloom et. al., 1997). Of those who applied for training, some were assigned training and some were assigned no training.

Because assignment is random and affects the level of treatment, one can treat assignment as an exclusion restriction that is correlated with treatment (i.e., the probability that $J_{i}=f$ ) but is uncorrelated with preferences or ability because it is random. In this sense, random assignment solves the selection problem that is the focus of the Roy model. As we show below, exogenous variation provided by experiments allows the researcher to cleanly identify some properties of the distribution of $Y_{f i}$ and $Y_{h i}$ under relatively weak assumptions. Furthermore, the methods for estimating these objects are simple, which adds to their appeal.

The treatment effect framework is also widely used for evaluating quasi-experimental data as well. By quasi-experimental data, we mean data that are not experimental, but exploit variation that is "almost as good as" random assignment.

\subsection{Treatment Effects and the Generalized Roy Model}

Within the context of the generalized Roy model note that in general

$$
\pi_{i}=g_{f}\left(X_{f i}, X_{0 i}\right)-g_{h}\left(X_{h i}, X_{0 i}\right)+\varepsilon_{f i}-\varepsilon_{h i}
$$

An important special case of the treatment effect defined in equation (5.1) is when

$$
\begin{aligned}
g_{f}\left(X_{f i}, X_{0 i}\right) & =g_{h}\left(X_{h i}, X_{0 i}\right)+\pi_{0} \\
\varepsilon_{f i} & =\varepsilon_{h i} .
\end{aligned}
$$

In this case, the treatment effect $\pi_{i}=Y_{f i}-Y_{h i}=\pi_{0}$ is a constant across individuals. Identification of this parameter is relatively straightforward. However, there is a substantial literature that studies identification of heterogeneous treatment effects. As we 
point out above, treatment effects are positive for some people and negative for others in the context of the Roy model. Furthermore, there is ample empirical evidence that the returns to job training are not constant, but instead vary across the population (Heckman, LaLonde, and Smith, 1999).

In section 4.2 we explain why the joint distribution of $\left(\varepsilon_{f i}, \varepsilon_{h i}\right)$ is not identified. This means that the distribution of $\pi_{i}$ is not identified and even relatively simple summary statistics like the median of this distribution is not identified in general. The key problem is that even when assignment is random, we do not observe the same people in both occupations.

Since the full generalized Roy model is complicated, hard to describe, and very demanding in terms of data, researchers often focus on a summary statistic to summarize the result. The most common in this literature is the Average Treatment Effect (ATE) defined as

$$
\begin{aligned}
A T E & \equiv E\left(\pi_{i}\right) \\
& =E\left(Y_{f i}\right)-E\left(Y_{h i}\right) .
\end{aligned}
$$

From Theorem 4.1 we know that (under the assumptions of that theorem) the distribution of $Y_{f i}$ and $Y_{h i}$ are identified. Thus, their expected values are also identified under the one additional assumption that these expected values exist.

Assumption 5.1. The expected values of $Y_{f i}$ and $Y_{h i}$ are finite

Theorem 5.1. Under the assumptions of Theorem 4.1 and Assumption 5.1, the Average Treatment effect is identified.

\section{(Proof in Appendix)}

To see where identification of this object comes from, abstract from $X_{i}$ so that the only observable is $Z_{i}$, which affects the non-pecuniary gain in utility from occupation across occupations. With experimental data, $Z_{i}$ could be randomly generated assignments to occupation. Notice that

$$
\begin{aligned}
& \lim _{\varphi(z) \rightarrow 1} E\left(Y_{f i} \mid Z_{i}=z, J_{i}=f\right)-\lim _{\varphi(z) \rightarrow 0} E\left(Y_{h i} \mid Z_{i}=z, J_{i}=h\right) \\
& =\lim _{\varphi(z) \rightarrow 1} E\left(Y_{f i} \mid \nu_{i} \leq \varphi(z)\right)-\lim _{\varphi(z) \rightarrow 0} E\left(Y_{h i} \mid \nu_{i}>\varphi(z)\right) \\
& =E\left(Y_{f i}\right)-E\left(Y_{h i}\right) .
\end{aligned}
$$


Thus the exclusion restriction is the key to identification. Note also that we need groups of individuals where $\varphi\left(Z_{i}\right) \approx 1$ (who are always fishermen) and $\varphi\left(Z_{i}\right) \approx 0$ (who are always hunters); thus "identification at infinity" is essential as well. For the reasons discussed in the nonparametric Roy model above, if $\varphi\left(Z_{i}\right)$ were never higher than some $\varphi\left(z^{u}\right)<1$ then $E\left(Y_{f i}\right)$ would not be identified. Similarly if $\varphi\left(Z_{i}\right)$ were never lower then some $\varphi\left(z^{\ell}\right)>0$, then $E\left(Y_{h i}\right)$ would not be identified.

While one could directly estimate the the ATE using "identification at infinity," as described above this is not the common practice and not something we would advocate. The standard approach would be to estimate the full Generalized Roy Model and then use it to simulate the various treatment effects. This is often done using a completely parametric approach as in, for example, the classic paper by Willis and Rosen (1979). However, there are quite a few nonparametric alternatives as well, including construction of the Marginal Treatment effects as discussed in section 5.3 and 5.4 below.

As it turns out, even with experimental data, it is rarely the case that $\varphi\left(Z_{i}\right)$ is identically one or zero with positive probability. In the case of medicine, some people assigned the treatment do not take the treatment. In the training example, many people who are offered subsidized training decide not to undergo the training. Thus, when compliance with assignment is less than $100 \%$, we cannot recover the ATE. In Section 5.2 we discuss more precisely what we do recover when there is less than $100 \%$ compliance.

It is also instructive to relate the ATE to instrumental variables estimation. Let $Y_{i}$ be the outcome of interest

$$
Y_{i}=\left\{\begin{array}{ll}
Y_{f i} & \text { if } J_{i}=f \\
Y_{h i} & \text { if } J_{i}=h
\end{array},\right.
$$

and let $D_{f i}$ be a dummy variable indicating whether $J_{i}=f$. Consider estimating the model

$$
Y_{i}=\beta_{0}+\beta_{1} D_{f i}+u_{i}
$$

using instrumental variables with $Z_{i}$ as an instrument for $D_{f i}$. Assume that $Z_{i}$ is correlated with $D_{f i}$ but not with $Y_{f i}$ or $Y_{h i}$. Consider first the constant treatment effect model described in equations (5.2) and (5.3) so that $\pi_{i}=\pi_{0}$ for everyone in the 
population. In that case

$$
\begin{aligned}
Y_{i} & =Y_{f i} D_{f i}+Y_{h i}\left(1-D_{f i}\right) \\
& =Y_{h i}+D_{f i}\left(Y_{f i}-Y_{h i}\right) \\
& =Y_{h i}+D_{f i} \pi_{0}
\end{aligned}
$$

Then two stage least squares on the model above yields

$$
\begin{aligned}
\operatorname{plim} \widehat{\beta}_{1} & =\frac{\operatorname{Cov}\left(Z_{i}, Y_{i}\right)}{\operatorname{Cov}\left(Z_{i}, D_{f i}\right)} \\
& =\frac{\operatorname{Cov}\left(Z_{i}, Y_{h i}+D_{f i} \pi_{0}\right)}{\operatorname{Cov}\left(Z_{i}, D_{f i}\right)} \\
& =\frac{\operatorname{Cov}\left(Z_{i}, Y_{h i}\right)}{\operatorname{Cov}\left(Z_{i}, D_{f i}\right)}+\frac{\operatorname{Cov}\left(Z_{i}, \pi_{0} D_{f i}\right)}{\operatorname{Cov}\left(Z_{i}, D_{f i}\right)} \\
& =\pi_{0} .
\end{aligned}
$$

Thus in the constant treatment effect model, instrumental variables provides a consistent estimate of the treatment effect. However, this result does not carry over to heterogeneous treatment effects and the average treatment effects as Heckman (1997) shows. Following the expression above we get

$$
\begin{aligned}
\operatorname{plim} \widehat{\beta}_{1} & =\frac{\operatorname{Cov}\left(Z_{i}, Y_{h i}+D_{f i} \pi_{i}\right)}{\operatorname{Cov}\left(Z_{i}, D_{f i}\right)} \\
& =\frac{\operatorname{Cov}\left(Z_{i}, D_{f i} \pi_{i}\right)}{\operatorname{Cov}\left(Z_{i}, D_{f i}\right)} \\
& \neq A T E
\end{aligned}
$$

in general. In sections 5.2 and 5.3 below, we describe what instrumental variables identifies.

In practice there are two potential problems with the assumptions behind Theorem 5.1 above

- The researcher may not have a valid exclusion restriction. We discuss some of the options for this case in Sections 5.5, 5.6, and 5.7.

- Even if they do, the variable may not have full support. By this we mean that the instrumental variable $Z_{i}$ may not vary enough so that for some observed values of $Z_{i}$ everyone is always a fisherman and for other observed values of $Z_{i}$ everyone 
is always a hunter. We discuss what can be identified using exclusion restrictions with limited support in Sections 5.2, 5.3, 5.4, and 5.6.

We discuss a number of different approaches some of which assumes an exclusion restriction but relaxes the support conditions and others that do not require exclusion restrictions.

\subsection{Local Average Treatment Effects}

Imbens and Angrist (1994) and Angrist, Imbens, and Rubin (1996) consider identification when the support of $Z_{i}$ takes on a finite number of points. They show that when varying the instrument over this range, they can identify what they call a Local Average Treatment Effect. Furthermore, they show how instrumental variables can be used to estimate it. It is again easiest to think about this problem after abstracting from $X_{i}$, as it is straightforward to condition on these variables (see Imbens and Angrist, 1994, for details). For simplicity's sake, consider the case in which the instrument $Z_{i}$ is binary and takes on the values $\{0,1\}$. In many cases not only is the instrument discrete, but it is also binary. For example, in randomized medical trials, $Z_{i}=1$ represents assignment to treatment, whereas $Z_{i}=0$ represents assignment to the placebo. In job training programs, $Z_{i}=1$ represents assignment to the training program, whereas $Z_{i}=0$ represents no assigned training.

It is important to point out that not all patients assigned treatment actually receive the treatment. Thus $J_{i}=f$ if the patient actually takes the drug and $J_{i}=h$ if the individual does not take the drug. Likewise, not all individuals who are assigned training actually receive the training, so $J_{i}=f$ if the individual goes to training and $J_{i}=h$ if she does not. The literature on Local Average Treatment Effects handles this case as well as many others. However, we do require that the instrument of assignment has power: $\operatorname{Pr}\left(J_{i}=f \mid Z_{i}=1\right) \neq \operatorname{Pr}\left(J_{i}=f \mid Z_{i}=0\right)$. Without loss of generality we will assume that $\operatorname{Pr}\left(J_{i}=f \mid Z_{i}=1\right)>\operatorname{Pr}\left(J_{i}=f \mid Z_{i}=0\right)$.

Using the reduced form version of the generalized Roy model the choice problem is

$$
J_{i}=f \text { if } \varphi\left(Z_{i}\right)>\nu_{i}
$$

where $\nu_{i}$ is uniformly distributed. 
The following six objects can be learned directly from the data:

$$
\begin{aligned}
& \operatorname{Pr}\left(J_{i}=f \mid Z_{i}=0\right)=\operatorname{Pr}\left(\nu_{i} \leq \varphi(0)\right) \\
& \operatorname{Pr}\left(J_{i}=f \mid Z_{i}=1\right)=\operatorname{Pr}\left(\nu_{i} \leq \varphi(1)\right) \\
& E\left(Y_{f i} \mid Z_{i}=0, J_{i}=f\right)=E\left(Y_{f i} \mid \nu_{i} \leq \varphi(0)\right) \\
& E\left(Y_{h i} \mid Z_{i}=0, J_{i}=h\right)=E\left(Y_{h i} \mid \nu_{i}>\varphi(0)\right) \\
& E\left(Y_{f i} \mid Z_{i}=1, J_{i}=f\right)=E\left(Y_{f i} \mid \nu_{i} \leq \varphi(1)\right) \\
& E\left(Y_{h i} \mid Z_{i}=1, J_{i}=h\right)=E\left(Y_{h i} \mid \nu_{i}>\varphi(1)\right),
\end{aligned}
$$

The above equations show that our earlier assumption that $\operatorname{Pr}\left(J_{i}=f \mid Z_{i}=1\right)>$ $\operatorname{Pr}\left(J_{i}=f \mid Z_{i}=0\right)$ implies $\operatorname{Pr}\left(\nu_{i} \leq \varphi(1)\right)>\operatorname{Pr}\left(\nu_{i} \leq \varphi(0)\right)$. This, combined with the structure embedded in equation (5.6) means that

$$
\operatorname{Pr}\left(\nu_{i} \leq \varphi(1) \mid \nu_{i} \leq \varphi(0)\right)=1
$$

so then an individual who is a fisherman when $Z_{i}=0$ is also a fisherman when $Z_{i}=1$. Similar reasoning implies $\operatorname{Pr}\left(\nu_{i} \leq \varphi(1) \mid \varphi(0)<\nu_{i} \leq \varphi(1)\right)=1$. Using this and Bayes rule yields

$$
\begin{aligned}
\operatorname{Pr}\left(\nu_{i} \leq \varphi(0) \mid \nu_{i} \leq \varphi(1)\right) & =\frac{\operatorname{Pr}\left(\nu_{i} \leq \varphi(1) \mid \nu_{i} \leq \varphi(0)\right) \operatorname{Pr}\left(\nu_{i} \leq \varphi(0)\right)}{\operatorname{Pr}\left(\nu_{i} \leq \varphi(1)\right)} \\
& =\frac{\operatorname{Pr}\left(\nu_{i} \leq \varphi(0)\right)}{\operatorname{Pr}\left(\nu_{i} \leq \varphi(1)\right)} \\
\operatorname{Pr}\left(\varphi(0)<\nu_{i} \leq \varphi(1) \mid \nu_{i} \leq \varphi(1)\right) & =\frac{\operatorname{Pr}\left(\nu_{i} \leq \varphi(1) \mid \varphi(0)<\nu_{i} \leq \varphi(1)\right) \operatorname{Pr}\left(\varphi(0)<\nu_{i} \leq \varphi(1)\right)}{\operatorname{Pr}\left(\nu_{i} \leq \varphi(1)\right)} \\
& =\frac{\operatorname{Pr}\left(\varphi(0)<\nu_{i} \leq \varphi(1)\right)}{\operatorname{Pr}\left(\nu_{i} \leq \varphi(1)\right)}
\end{aligned}
$$

Using the fact that $\operatorname{Pr}\left(\nu_{i} \leq \varphi(1)\right)=\operatorname{Pr}\left(\nu_{i} \leq \varphi(0)\right)+\operatorname{Pr}\left(\varphi(0)<\nu_{i} \leq \varphi(1)\right)$, one can show that

$$
\begin{aligned}
E\left(Y_{f i} \mid \nu_{i} \leq \varphi(1)\right)= & E\left(Y_{f i} \mid \nu_{i} \leq \varphi(0)\right) \operatorname{Pr}\left(\nu_{i} \leq \varphi(0) \mid \nu_{i} \leq \varphi(1)\right) \\
& +E\left(Y_{f i} \mid \varphi(0)<\nu_{i} \leq \varphi(1)\right) \operatorname{Pr}\left(\varphi(0)<\nu_{i} \leq \varphi(1) \mid \nu_{i} \leq \varphi(1)\right)
\end{aligned}
$$


Combining equation (5.10) with equations (5.8) and (5.9) yields

$$
\begin{aligned}
E\left(Y_{f i} \mid \nu_{i} \leq \varphi(1)\right)= & \frac{E\left(Y_{f i} \mid \nu_{i} \leq \varphi(0)\right) \operatorname{Pr}\left(\nu_{i} \leq \varphi(0)\right)}{\operatorname{Pr}\left(\nu_{i} \leq \varphi(1)\right)} \\
& +\frac{E\left(Y_{f i} \mid \varphi(0)<\nu_{i} \leq \varphi(1)\right) \operatorname{Pr}\left(\varphi(0)<\nu_{i} \leq \varphi(1)\right)}{\operatorname{Pr}\left(\nu_{i} \leq \varphi(1)\right)}
\end{aligned}
$$

Rearranging equation (5.11) shows that we can identify

$$
\begin{aligned}
& E\left(Y_{f i} \mid \varphi(0) \leq \nu_{i}<\varphi(1)\right) \\
& =\frac{E\left(Y_{f i} \mid Z_{i}=1, J_{i}=f\right) \operatorname{Pr}\left(J_{i}=f \mid Z_{i}=1\right)-E\left(Y_{f i} \mid Z_{i}=0, J_{i}=f\right) \operatorname{Pr}\left(J_{i}=f \mid Z_{i}=0\right)}{\operatorname{Pr}\left(J_{i}=f \mid Z_{i}=1\right)-\operatorname{Pr}\left(J_{i}=f \mid Z_{i}=0\right)}
\end{aligned}
$$

since everything on the right hand side is directly identified from the data.

Using the analogous argument one can show that

$$
\begin{aligned}
& E\left(Y_{h i} \mid \varphi(0) \leq \nu_{i}<\varphi(1)\right) \\
& \quad=\frac{E\left(Y_{h i} \mid Z_{i}=0, J_{i}=h\right) \operatorname{Pr}\left(J_{i}=h \mid Z_{i}=0\right)-E\left(Y_{h i} \mid Z_{i}=1, J_{i}=h\right) \operatorname{Pr}\left(J_{i}=h \mid Z_{i}=1\right)}{\operatorname{Pr}\left(J_{i}=f \mid Z_{i}=1\right)-\operatorname{Pr}\left(J_{i}=f \mid Z_{i}=0\right)}
\end{aligned}
$$

is identified. But this means that we can identify

$$
E\left(\pi_{i} \mid \varphi(0) \leq \nu_{i}<\varphi(1)\right)=E\left(Y_{f i}-Y_{h i} \mid \varphi(0) \leq \nu_{i}<\varphi(1)\right)
$$

which Imbens and Angrist (1994) define as the Local Average Treatment Effect. This is the average treatment effect for that group of individuals who would alter their treatment status if their value of $Z_{i}$ changed. Given the variation in $Z_{i}$, this is the only group for whom we can identify a treatment effect. Any individual in the data with $\nu_{i}>\varphi(1)$ would never choose $J_{i}=f$, so the data are silent about $E\left(Y_{f i} \mid \nu_{i}>\varphi(1)\right)$. Similarly the data is silent about $E\left(Y_{h i} \mid \nu_{i} \leq \varphi(0)\right)$.

Imbens and Angrist (1994) also show that the standard linear Instrumental Variables estimator yield consistent estimates of Local Average Treatment Effects. Consider the instrumental variables estimator of equation (5.4)

$$
Y_{i}=\beta_{0}+\beta_{1} D_{f i}+u_{i}
$$

In equation (5.5) we showed that

$$
\begin{aligned}
\widehat{\beta}_{1} \stackrel{p}{\rightarrow} & \frac{\operatorname{Cov}\left(Z_{i}, D_{f i} \pi_{i}\right)}{\operatorname{Cov}\left(Z_{i}, D_{f i}\right)} \\
& =\frac{E\left(\pi_{i} D_{f i} Z_{i}\right)-E\left(\pi_{i} D_{f i}\right) E\left(Z_{i}\right)}{E\left(D_{f i} Z_{i}\right)-E\left(D_{f i}\right) E\left(Z_{i}\right)}
\end{aligned}
$$


Let $P_{z}$ denote the probability that $Z_{i}=1$. The numerator of the above expression is

$$
\begin{aligned}
& E\left(\pi_{i} D_{f i} Z_{i}\right)-E\left(\pi_{i} D_{f i}\right) E\left(Z_{i}\right) \\
= & P_{z} E\left(\pi_{i} D_{f i} \mid Z_{i}=1\right)-E\left(\pi_{i} D_{f i}\right) P_{z} \\
= & P_{z} E\left(\pi_{i} D_{f i} \mid Z_{i}=1\right)-\left[P_{z} E\left(\pi_{i} D_{f i} \mid Z_{i}=1\right)+\left(1-P_{z}\right) E\left(\pi_{i}, D_{f i} \mid Z_{i}=0\right)\right] P_{z} \\
= & P_{z}\left(1-P_{z}\right)\left[E\left(\pi_{i} D_{f i} \mid Z_{i}=1\right)-E\left(\pi_{i} D_{f i} \mid Z_{i}=0\right)\right] \\
= & P_{z}\left(1-P_{z}\right) E\left(\pi_{i} \mid \varphi(0)<\nu_{i} \leq \varphi(1)\right) \operatorname{Pr}\left(\varphi(0)<\nu_{i} \leq \varphi(1)\right)
\end{aligned}
$$

where the key simplification comes from the fact that

$$
\begin{aligned}
E\left(\pi_{i} D_{f i} \mid Z_{i}=1\right) & =E\left(\pi_{i} 1\left(\nu_{i} \leq \varphi(1)\right)\right) \\
& =E\left(\pi_{i}\left[1\left(\nu_{i} \leq \varphi(0)\right)+1\left(\varphi(0)<\nu_{i} \leq \varphi(1)\right)\right]\right) \\
& =E\left(\pi_{i} D_{f i} \mid Z_{i}=0\right)+E\left(\pi_{i} \mid \varphi(0)<\nu_{i} \leq \varphi(1)\right) \operatorname{Pr}\left(\varphi(0)<\nu_{i} \leq \varphi(1)\right) .
\end{aligned}
$$

Next consider the denominator

$$
\begin{aligned}
& E\left(D_{f i} Z_{i}\right)-E\left(D_{f i}\right) E\left(Z_{i}\right) \\
= & P_{z} E\left(D_{f i} \mid Z_{i}=1\right)-E\left(D_{f i}\right) P_{z} \\
= & P_{z} E\left(D_{f i} \mid Z_{i}=1\right)-\left[P_{z} E\left(D_{f i} \mid Z_{i}=1\right)+\left(1-P_{z}\right) E\left(D_{f i} \mid Z_{i}=0\right)\right] P_{z} \\
= & P_{z}\left(1-P_{z}\right)\left[E\left(D_{f i} \mid Z_{i}=1\right)-E\left(D_{f i} \mid Z_{i}=0\right)\right] \\
= & P_{z}\left(1-P_{z}\right) \operatorname{Pr}\left(\varphi(0)<\nu_{i} \leq \varphi(1)\right)
\end{aligned}
$$

Thus

$$
\begin{aligned}
\widehat{\beta}_{1} \stackrel{p}{\rightarrow} & \frac{E\left(\pi_{i} D_{f i} Z_{i}\right)-E\left(\pi_{i} D_{f i}\right) E\left(Z_{i}\right)}{E\left(D_{f i} Z_{i}\right)-E\left(D_{f i}\right) E\left(Z_{i}\right)} \\
& =\frac{P_{z}\left(1-P_{z}\right) E\left(\pi_{i} \mid \varphi(0)<\nu_{i} \leq \varphi(1)\right) \operatorname{Pr}\left(\varphi(0)<\nu_{i} \leq \varphi(1)\right)}{P_{z}\left(1-P_{z}\right) \operatorname{Pr}\left(\varphi(0)<\nu_{i} \leq \varphi(1)\right)} \\
& =E\left(\pi_{i} \mid \varphi(0)<\nu_{i} \leq \varphi(1)\right)
\end{aligned}
$$

Imbens and Angrist never explicitly use the generalized Roy model or the latent index framework. Instead, they write their problem only in terms of the choice probabilities. However, in order to do this they must make one additional assumption. Specifically, they assume that if $J_{i}=f$ when $Z_{i}=0$ then $J_{i}=f$ when $Z_{i}=1$. Thus 
changing $Z_{i}=0$ to $Z_{i}=1$ never causes some people to switch from fishing to hunting. It only causes people to switch from hunting to fishing. They refer to this as a monotonicity assumption. Vytlacil (2002) points out that this is implied by the latent index model when the index $\varphi\left(Z_{i}\right)$ is separable from $\nu_{i}$ as we assumed in equation (5.6). As is implied by equation (5.7), increasing the index $\varphi\left(Z_{i}\right)$ will cause some people to switch from hunting to fishing, but not the reverse. ${ }^{14}$

Throughout, we use the latent index framework that is embedded in the Generalized Roy model, for three reasons. First, we can appeal to the identification results of the Generalized Roy model. Second, the latent index can be interpreted as the added utility from making a decision. Thus we can use the estimated model for welfare analysis. Third, placing the choice in an optimizing framework allows us to test the restrictions on choice that come from the theory of optimization.

As we have pointed out, not everyone offered training actually takes the training. For example, in the case of the JTPA, only $60 \%$ of those offered the training actually received it (Bloom et al., 1997). Presumably, those who took the training are those who stood the most to gain from the training. For example, the reason that many people do not take training is that they receive a job offer before training begins. For these people, the training may have been of relatively little value. Furthermore, $2 \%$ of those who applied for and were not assigned training program wind up receiving the training (Bloom et al., 1997). Angrist, Imbens and Rubin (1996) refer to those who were assigned training, but did not take the training as never-takers. Those who receive the training whether or not they are assigned are always-takers. Those who receive the training only when assigned the training are compliers. In terms of the latent index framework, the never-takers are those for whom $\left(\nu_{i} \geq \varphi(1)\right)$, the compliers are those for whom $\left(\varphi(0) \leq \nu_{i}<\varphi(1)\right)$, and the always-takers are those for whom $\left(\nu_{i}<\varphi(0)\right)$.

The monotonicity assumption embedded in the latent index framework rules out the existence of a final group: the defiers. In the context of training, this would be an individual who receives training when not assigned training but would not receive training when assigned. At least in the context of training programs (and many other contexts) it seems safe to assume that there are no defiers.

\footnotetext{
${ }^{14}$ However, he points out that the non-separable model $D_{f i}=1\left(f\left(Z_{i}, \nu_{i}\right)>0\right)$ does not necessarily give rise to monotonicity. All other differences between the latent variable framework and the LATE framework are extremely technical and minor.
} 


\subsection{Marginal Treatment Effects}

Heckman and Vytlacil (1999, 2001, 2005, 2007b) develop a framework that is useful for constructing many types of treatment effects. They focus on the marginal treatment effect (MTE) defined in our context as

$$
\Delta^{M T E}(x, \nu) \equiv E\left(\pi_{i} \mid X_{i}=x, \nu_{i}=\nu\right)
$$

They show formally how to identify this object. We present their methodology using our notation.

Note that if we allow for regressors $X_{i}$, let the exclusion restriction $Z_{i}$ to take on values beyond zero and one, then if $\left(z^{\ell}, x\right)$ and $\left(z^{h}, x\right)$ are in the support of the data, then equation (5.12) can be rewritten as

$$
\begin{aligned}
& E\left(Y_{f i} \mid \varphi\left(z^{\ell}, x\right) \leq \nu_{i}<\varphi\left(z^{h}, x\right), X_{i}=x\right) \\
= & \frac{E\left(Y_{f i} \mid\left(Z_{i}, X_{i}\right)=\left(z^{h}, x\right), J_{i}=f\right) \operatorname{Pr}\left(J_{i}=f \mid\left(Z_{i}, X_{i}\right)=\left(z^{h}, x\right)\right)}{\operatorname{Pr}\left(J_{i}=f \mid\left(Z_{i}, X_{i}\right)=\left(z^{h}, x\right)\right)-\operatorname{Pr}\left(J_{i}=f \mid\left(Z_{i}, X_{i}\right)=\left(z^{\ell}, x\right)\right)} \\
& -\frac{E\left(Y_{f i} \mid\left(Z_{i}, X_{i}\right)=\left(z^{\ell}, x\right), J_{i}=f\right) \operatorname{Pr}\left(J_{i}=f \mid\left(Z_{i}, X_{i}\right)=\left(z^{\ell}, x\right)\right)}{\operatorname{Pr}\left(J_{i}=f \mid\left(Z_{i}, X_{i}\right)=\left(z^{h}, x\right)\right)-\operatorname{Pr}\left(J_{i}=f \mid\left(Z_{i}, X_{i}\right)=\left(z^{\ell}, x\right)\right)}
\end{aligned}
$$

for $\varphi\left(z^{\ell}, x\right)<\varphi\left(z^{h}, x\right)$. Now notice that for any $\nu$,

$$
\lim _{\varphi\left(z^{\ell}, x\right) \uparrow \nu, \varphi\left(z^{h}, x\right) \downarrow \nu} E\left(Y_{f i} \mid \varphi\left(z^{\ell}, x\right) \leq \nu_{i}<\varphi\left(z^{h}, x\right), X_{i}=x\right)=E\left(Y_{f i} \mid \nu_{i}=\nu, X_{i}=x\right) .
$$

Thus if $(x, \nu)$ is in the support of $\left(X_{i}, \varphi\left(Z_{i}, X_{i}\right)\right)$, then $E\left(Y_{f i} \mid \nu_{i}=\nu, X_{i}=x\right)$ is identified. Since the model is symmetric, under similar conditions $E\left(Y_{h i} \mid \nu_{i}=\nu, X_{i}=\right.$ $x$ ) is identified as well. Finally since

$$
\begin{aligned}
\Delta^{M T E}(x, \nu) & =E\left(\pi_{i} \mid X_{i}=x, \nu_{i}=\nu\right) \\
& =E\left(Y_{f i} \mid \nu_{i}=\nu, X_{i}=x\right)-E\left(Y_{h i} \mid \nu_{i}=\nu, X_{i}=x\right),
\end{aligned}
$$

the marginal treatment effect is identified.

The marginal treatment effect is interesting in its own right. It is the value of the treatment for any individual with $X_{i}=x$ and $\nu_{i}=\nu$. In addition, it is also useful because the different types of treatment effects can be defined in terms of the marginal treatment effect. For example

$$
A T E=\iint_{0}^{1} \Delta^{M T E}(x, \nu) d \nu d G(x) .
$$


One can see from this expression that without full support this will not be identified because $\Delta^{M T E}(x, \nu)$ will not be identified everywhere.

Heckman and Vytlacil (2005) also show that the instrumental variables estimator defined in equation (5.5) (conditional on $x$ ) is

$$
\int_{0}^{1} \Delta^{M T E}(x, \nu) h_{I V}(x, \nu) d \nu
$$

where they give an explicit functional form for $h_{I V}$. It is complicated enough that we do not repeat it here but it can be found in Heckman and Vytlacil (2005).

This framework is also useful for seeing what is not identified. In particular if $\varphi\left(Z_{i}, x\right)$ does not have full support so that it is bounded above or below the average treatment effect will not be identified. However, many other interesting treatment effects can be identified. For example, the Local Average Treatment Effect in a model with no regressors $(x)$ is

$$
L A T E=\frac{\int_{\varphi(0)}^{\varphi(1)} \Delta^{M T E}(\nu) d \nu}{\varphi(1)-\varphi(0)} .
$$

More generally, in this series of papers, Heckman and Vytlacil show that the marginal treatment effect can also be used to organize many ideas in the literature. One interesting case is policy effects. They define the policy relevant treatment effect as the treatment resulting from a particular policy. They show that if the relationship between the policy and the observable covariates is known, the policy relevant treatment effect can be identified from the marginal treatment effects.

\subsection{Applications of the Marginal Treatment Effects Approach}

Heckman and Vytlacil $(1999,2001,2005)$ suggest procedures to estimate the marginal treatment effect. They suggest what they call "local instrumental variables." Using our notation for the generalized Roy model in which $J_{i}=f$ when $\varphi\left(X_{i}, Z_{i}\right)-\nu_{i}>0$ where $\nu_{i}$ is uniformly distributed, they show that

$$
\Delta^{M T E}(x, \nu)=\frac{\partial E\left(Y_{i} \mid X_{i}=x, \varphi\left(X_{i}, Z_{i}\right)=\nu\right)}{\partial \nu}
$$


To see why this is the same definition of MTE as in equation (5.15), note that

$$
\begin{aligned}
& \frac{\partial E\left(Y_{i} \mid X_{i}=x, \varphi\left(X_{i}, Z_{i}\right)=\nu\right)}{\partial \nu} \\
& =\frac{\partial\left[E\left(Y_{f i} \mid X_{i}=x, \nu_{i} \leq \nu\right) \operatorname{Pr}\left(\nu_{i} \leq \nu\right)+E\left(Y_{h i} \mid X_{i}=x, \nu_{i}>\nu\right) \operatorname{Pr}\left(\nu_{i}>\nu\right)\right]}{\partial \nu} \\
& =\frac{\partial\left[\int_{0}^{\nu} E\left(Y_{f i} \mid \nu_{i}=\omega, X_{i}=x\right) d \omega+\int_{\nu}^{1} E\left(Y_{h i} \mid \nu_{i}=\omega, X_{i}=x\right) d \omega\right]}{\partial \nu} \\
& =E\left(Y_{f i} \mid \nu_{i}=\nu, X_{i}=x\right)-E\left(Y_{h i} \mid \nu_{i}=\nu, X_{i}=x\right) \\
& =\Delta^{M T E}(x, \nu) .
\end{aligned}
$$

Thus one can estimate the marginal treatment effect in three steps. First estimate $\varphi$, second estimate $E\left(Y \mid X_{i}=x, \varphi\left(X_{i}, Z_{i}\right)=\nu\right)$ using some type of nonparametric regression approach, and third take the derivative.

Because as a normalization $\nu_{i}$ is uniformly distributed

$$
\begin{aligned}
\varphi(x, z) & =\operatorname{Pr}\left(\nu_{i} \leq \varphi\left(X_{i}, Z_{i}\right) \mid X_{i}=x, Z_{i}=z\right) \\
& =\operatorname{Pr}\left(J_{i}=f \mid X_{i}=x, Z_{i}=z\right) \\
& =E\left(D_{f i} \mid X_{i}=x, Z_{i}=z\right) .
\end{aligned}
$$

Thus we can estimate $\varphi(x, z)$ from a nonparametric regression of $D_{f i}$ on $\left(X_{i}, Z_{i}\right)$.

A very simple way to do this is to use a linear probability model of $D_{f i}$ regressed on a polynomial of $Z_{i}$. By letting the terms in the polynomial get large with the sample size, this can be considered a nonparametric estimator. For the second stage we regress the outcome $Y_{i}$ on a polynomial of our estimate of $\varphi\left(Z_{i}\right)$. To see how this works consider the case in which both polynomials are quadratics. We would use the following two stage least squares procedure:

$$
\begin{gathered}
D_{f i}=\gamma_{0}+\gamma_{1} Z_{i}+\gamma_{2} Z_{i}^{2}+\gamma_{x} X_{i}+e_{i}, \\
Y_{i}=\beta_{0}+\beta_{1} \widehat{D_{f i}}+\beta_{2}{\widehat{D_{f i}}}^{2}+\beta_{x} X_{i}+u_{i},
\end{gathered}
$$

where $\widehat{D_{f i}}=\widehat{\gamma_{0}}+\widehat{\gamma_{1}} Z_{i}+\widehat{\gamma_{2}} Z_{i}^{2}+\widehat{\gamma_{x}} X_{i}$ is the predicted value from the first stage. The $\beta_{2}$ coefficient may not be 0 because as we change $\widehat{D_{f i}}$ the instrument affects different groups of people. The MTE is the effect of changing $\widehat{D_{f i}}$ on $Y_{i}$. For the case above the MTE is:

$$
\frac{\partial Y_{i}}{\partial \widehat{D_{f i}}}=\beta_{1}+2 \beta_{2} \widehat{D_{f i}}
$$


Although the polynomial procedure above is transparent, the most common technique used to estimate the MTE is local linear regression.

French and Song (2010) estimate the labor supply response to Disability Insurance (DI) receipt for DI applicants. Individuals are deemed eligible for DI benefits if they are "unable to engage in substantial gainful activity"-i.e., if they are unable to work. Beneficiaries receive, on average $\$ 12,000$ per year, plus Medicare health insurance. Thus, there are strong incentives to apply for benefits. They continue to receive these benefits only if they earn less than a certain amount per year $(\$ 10,800$ in 2007). For this reason, the DI system likely has strong labor supply disincentives. A healthy DI recipient is unlikely to work if that causes the loss of DI and health insurance benefits.

The DI system attempts to allow benefits only to those who are truly disabled. Many DI applicants have their case heard by a judge who determines those who are truly disabled. Some applicants appear more disabled than others. The most disabled applicants are unable to work, and thus will not work whether or not they get the benefit. For less serious cases, the applicant will work, but only if she is denied benefits. The question, then, is what is the optimal threshold level for the amount of observed disability before the individual is allowed benefits? Given the definition of disability, this threshold should depend on the probability that an individual does not work, even when denied the benefit. Furthermore, optimal taxation arguments suggest that benefits should be given to groups whose labor supply is insensitive to benefit allowance. Thus the effect of DI allowance on labor supply is of great interest to policy makers.

OLS is likely to be inconsistent because those who are allowed benefits are likely to be less healthy than those who are denied. Those allowed benefits would have had low earnings even if they did not receive benefits. French and Song propose an IV estimator using the process of assignment of cases to judges. Cases are assigned to judges on a rotational basis within each hearing office, which means that for all practical purposes, judges are randomly assigned to cases conditional on the hearing office and the day. Some judges are much more lenient than others. For example, the least lenient 5\% of all judges allow benefits to less than $45 \%$ of the cases they hear, whereas the most lenient $5 \%$ of all judges allow benefits to $80 \%$ of all the cases they hear. Although some of those who are denied benefits appeal and get benefits later, most do not. If assignment of cases to judges is random then the instrument of judge assignment is a 
plausibly exogenous instrument. Furthermore, and as long as judges vary in terms of leniency and not ability to detect individuals who are disabled, ${ }^{15}$ the instrument can identify a MTE.

French and Song use a two stage procedure. In the first stage they estimate the probability that an individual is allowed benefits, conditional on the average judge specific allowance rate. They estimate a version of equation (5.17) where $D_{f i}$ is an indicator equal to 1 if case $i$ was allowed benefits and $Z_{i}$ is the average allowance rate of the judge who heard case $i$. In the second stage they estimate earnings conditional on whether the individual was allowed benefits (as predicted by the judge specific allowance rate). They estimate a version of equation (5.18) where $Y_{i}$ is annual earnings 5 years after assignment to a judge. Figure 1 shows the estimated MTE (using the formula in equation (5.19)) using several different specifications of polynomial in the first and second stage equations. Assuming that the treatment effect is constant (i.e., $\beta_{2}=0$ ), they find that annual earnings 5 years after assignment to a judge are $\$ 1,500$ for those allowed benefits and $\$ 3,900$ for those denied benefits, so the estimated treatment effect is $\$ 2,400$. This is the MTE-linear case in Figure 1 . However, this masks considerable heterogeneity in the treatment effects. They find that when allowance rates rise, the labor supply response of the marginal case also rises. When allowing for the quadratic term $\beta_{2}$ to be non-zero, they find that less lenient judges (who allow $45 \%$ of all cases) have a MTE of a $\$ 1,800$ decline in earnings. More lenient judges (who allow $80 \%$ of all cases) have a MTE of $\$ 3,200$ decline in earnings. Figure 1 also shows results when allowing for cubic and quartic terms in the polynomials in the first and second stage equations. This result is consistent with the notion that as allowance rates rise, more healthy individuals are allowed benefits. These healthier individuals are more likely to work when not receiving DI benefits, and thus their labor supply response to DI receipt is greater.

One problem with an instrument such as this is that the instrument lacks full support. Even the most lenient judge does not allow everyone benefits. Even the strictest judge does not deny everyone. However, the current policy debate is whether the thresholds should be changed by only a modest amount. For this reason, the MTE

\footnotetext{
${ }^{15}$ If judges vary in terms of ability to detect disability, then a case that is allowed by a low allowance judge might be denied by a high allowance judge. This would violate the monotonicity assumption shown in equation (5.7).
} 


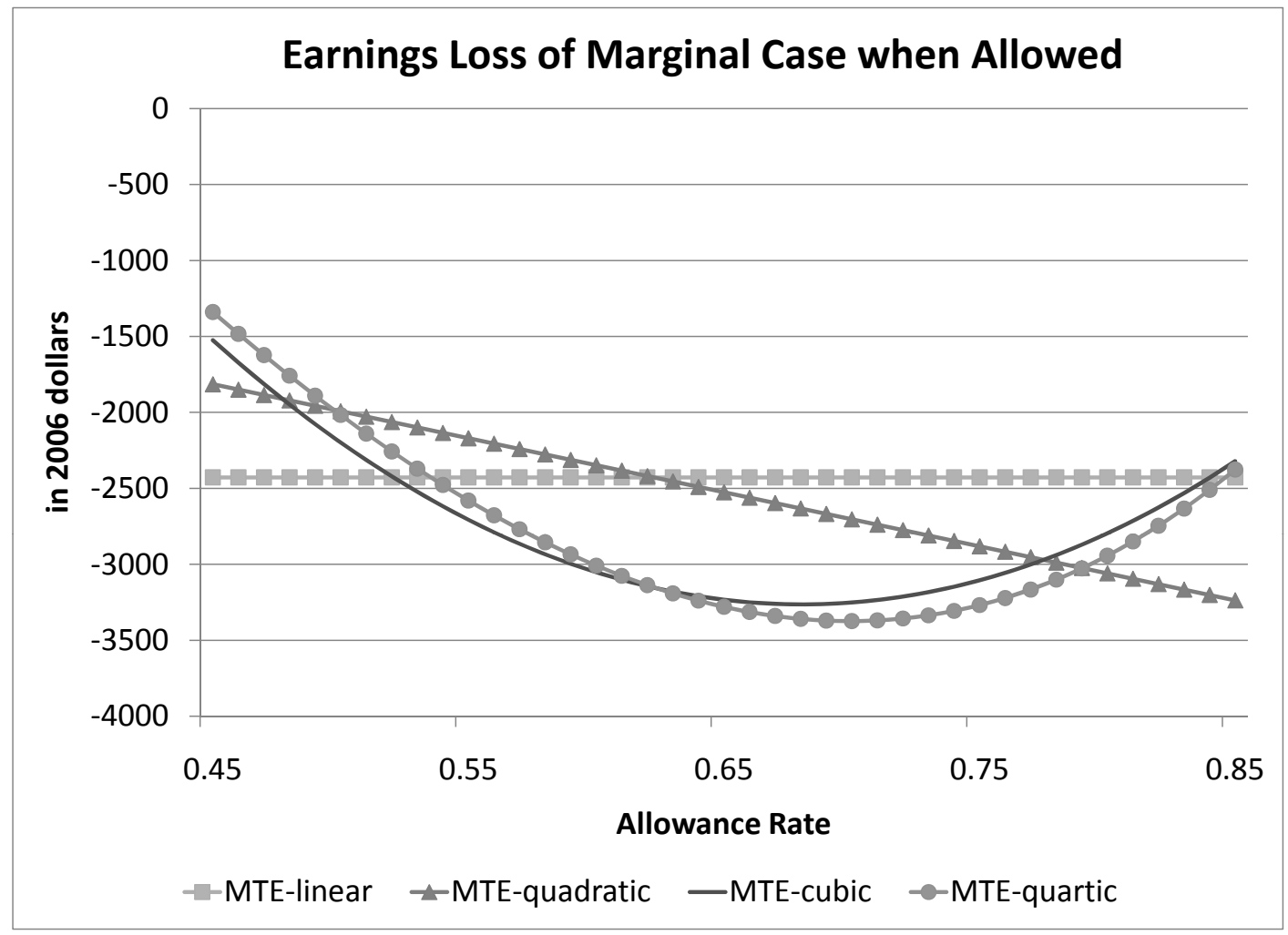

Figure 1: Marginal Treatment EfFect

on the support of the data is the effect of interest, whereas the ATE is not.

Doyle (2007) estimates the Marginal Treatment Effect of foster care on future earnings and other outcomes. Foster care likely increases earnings of some children but decreases it for others. For the most serious child abuse cases, foster care will likely help the child. For less serious cases, the child is probably best left at home. The question, then, is at what point should the child abuse investigator remove the child from the household? What is the optimal threshold level for the amount of observed abuse before which the child is removed from the household and placed into foster care?

Only children from the most disadvantaged backgrounds are placed in foster care. They would have had low earnings even if they were not placed in foster care. Thus, OLS estimates are likely inconsistent. To overcome this problem, Doyle uses IV. Case investigators are assigned to cases on a rotational basis, conditional on time and the 
location of the case. Case investigators are assigned to possible child abuse cases after a complaint of possible child abuse is made (by the child's teacher, for example). Investigators have a great deal of latitude about whether the child should be sent into foster care. Furthermore, some investigators are much more lenient than others. For example, one standard deviation in the case manager removal differential (the difference between his average removal rate and the removal rate of other investigators who handle cases at the same time and place) is $10 \%$. Whether the child is removed from the home is a good predictor of whether the child is sent to foster care. So long as assignment of cases to investigators is random and investigators only vary in terms of leniency (and not ability to detect child abuse) then the instrument of investigator assignment is a useful and plausibly exogenous instrument.

Doyle uses a two stage procedure where in the first stage he estimates the probability that a child is placed in foster care as a function of the investigator removal rate. In the second stage he estimates adult earnings as a function of whether the child was placed in foster care (as predicted by the instrument). He finds that children placed into foster care earn less than those not placed into foster care over most of the range of the data. Two stage least squares estimates reveal that foster care reduces adult quarterly earnings by about $\$ 1000$, which is very close to average earnings. Interestingly, he finds that when child foster care placement rates rise, earnings of the marginal case fall. For example, earnings of the marginal child handled by a lenient investigator (who places only $20 \%$ of the children in foster care) are unaffected by placement. For less lenient investigators, who place $25 \%$ of the cases in foster care, earnings of the marginal case decline by over $\$ 1500$.

Carneiro and Lee (2009) estimate the counterfactual marginal distributions of wages for college and high school graduates, and examine who enters college. They find that those with the highest returns are the most likely to attend college. Thus, increases in college cause changes in the distribution of ability among college and high school graduates. For fixed skill prices, they find that a $14 \%$ increase in college participation (analogous to the increase observed in the 1980s), reduces the college premium by $12 \%$. Likewise, Carneiro, Heckman and Vytlacil (2010) find that while the conventional IV estimate of the return to schooling (using distance to a college and local labor market conditions as the instruments) is 0.095 , the estimated marginal return to a policy that 
expands each individual's probability of attending college by the same proportion is only 0.015 .

\subsection{Selection on Observables}

Perhaps the simplest and most common assumption is that assignment of the treatment is random conditional on observable covariates (sometimes referred to as unconfoundedness). The easiest way to think about this is that the selection error term is independent of the other error terms:

\section{Assumption 5.2.}

$$
J_{i}=f \text { when } \varphi\left(X_{i}\right)>\nu_{i}
$$

where $\nu_{i}$ is independent of $\left(\varepsilon_{f i}, \varepsilon_{h i}\right)$.

We continue to assume that $Y_{f i}=g_{f}\left(X_{f i}, X_{0 i}\right)+\varepsilon_{f i}$ and $Y_{h i}=g_{h}\left(X_{h i}, X_{0 i}\right)+\varepsilon_{h i}$. Note that we have explicitly dropped $Z_{i}$ from the model as we consider cases in which we do not have exclusion restrictions. The implication of this assumption is that unobservable factors that determine one's income as a fisherman do not affect the choice to become a fisherman. That is while it allows for selection on observables in a very general way, it does not allow for selection on unobservables.

Interestingly, this is still not enough for us to identify the Average Treatment Effect. If there are values of observable covariates $X_{i}$ for which $\operatorname{Pr}\left(J_{i}=f \mid X_{i}=x\right)=1$ or $\operatorname{Pr}\left(J_{i}=f \mid X_{i}=x\right)=0$ the model is not identified. If $\operatorname{Pr}\left(J_{i}=f \mid X_{i}=x\right)=1$ then it is straight forward to identify $E\left(Y_{f i} \mid X_{i}=x\right)$, but $E\left(Y_{h i} \mid X_{i}=x\right)$ is not identified. Thus we need the additional assumption

Assumption 5.3. For almost all $x$ in the support of $X_{i}$,

$$
0<\operatorname{Pr}\left(J_{i}=f \mid X_{i}=x\right)<1
$$

Theorem 5.2. Under assumptions 5.2 and 5.3 the Average Treatment Effect is identified

(Proof in Appendix) 
Estimation in this case is relatively straightforward. One can use matching ${ }^{16}$ or regression analysis to estimate the average treatment effect.

\subsection{Set Identification of Treatment Effects}

In our original discussion of identification we defined $\Psi(\Theta(P))$ as "the set of values of $\psi$ that are consistent with the data distribution $P$." We said that $\psi$ was identified if this set was a singleton. However, there is another concept of identification we have not discussed until this point which is set identification. Sometimes we may be interested in a parameter that is not point identified, but this does not mean we cannot say anything about it. In this subsection we consider the case of set identification (i.e. trying to characterize the set $\Psi(\Theta(P)))$ focusing on the case in which $\psi$ is the Average Treatment Effect. Suppose that we have some prior knowledge (possibly an exclusion restriction that gives us a LATE). What can we learn about the ATE without making any functional form assumptions? In a series of papers Manski $(1989,1990,1995,1997)$ and Manski and Pepper $(2000,2009)$ develop procedures to derive set estimators of the Average Treatment Effect and other parameters given weak assumptions. By "set identification" we mean the set of possible Average Treatment Effects given the assumptions placed on the the data. Throughout this section we will continue to assume that the structure of the Generalized Roy model holds and we derive results under these assumptions. In many cases the papers we mentioned did not impose this structure and get more general results.

Following Manski (1990) or Manski (1995), notice that

$$
\begin{aligned}
& E\left(Y_{f i}\right)=E\left(Y_{f i} \mid J_{i}=f\right) \operatorname{Pr}\left(J_{i}=f\right)+E\left(Y_{f i} \mid J_{i}=h\right) \operatorname{Pr}\left(J_{i}=h\right) \\
& E\left(Y_{h i}\right)=E\left(Y_{h i} \mid J_{i}=h\right) \operatorname{Pr}\left(J_{i}=h\right)+E\left(Y_{h i} \mid J_{i}=f\right) \operatorname{Pr}\left(J_{i}=f\right) .
\end{aligned}
$$

We observe all of the objects in equations (5.20) and (5.21) except $E\left(Y_{f i} \mid J_{i}=h\right)$ and $E\left(Y_{h i} \mid J_{i}=f\right)$. The data are completely uninformative about these two objects. However, suppose we have some prior knowledge about the support of $Y_{f i}$ and $Y_{h i}$. In particular, suppose that the support of $Y_{f i}$ and $Y_{h i}$ are bounded above by $y^{u}$ and from

\footnotetext{
${ }^{16}$ Our focus is on identification rather than estimation. Thus we avoid a discussion of matching estimators. See Heckman, Lalonde, and Smith (1999), Imbens and Wooldridge (2009), or Dinardo and Lee (2010) for discussion.
} 
below by $y^{\ell}$. Thus, by assumption $y^{u} \geq E\left(Y_{f i} \mid J_{i}=h\right) \geq y^{\ell}$ and $y^{u} \geq E\left(Y_{h i} \mid J_{i}=\right.$ $f) \geq y^{\ell}$. Using these assumptions and equations (5.20) and (5.21) we can establish that

$$
\begin{aligned}
& E\left(Y_{f i} \mid J_{i}=f\right) \operatorname{Pr}\left(J_{i}=f\right)+y^{\ell} \operatorname{Pr}\left(J_{i}=h\right) \\
& \leq E\left(Y_{f i}\right) \leq E\left(Y_{f i} \mid J_{i}=f\right) \operatorname{Pr}\left(J_{i}=f\right)+y^{u} \operatorname{Pr}\left(J_{i}=h\right) \\
& E\left(Y_{h i} \mid J_{i}=h\right) \operatorname{Pr}\left(J_{i}=h\right)+y^{\ell} \operatorname{Pr}\left(J_{i}=f\right) \\
& \leq E\left(Y_{h i}\right) \leq E\left(Y_{h i} \mid J_{i}=h\right) \operatorname{Pr}\left(J_{i}=h\right)+y^{u} \operatorname{Pr}\left(J_{i}=f\right) .
\end{aligned}
$$

Using these bounds and the definition of the ATE

$$
A T E=E\left(Y_{f i}\right)-E\left(Y_{h i}\right)
$$

yields

$$
\begin{aligned}
& \left(E\left(Y_{f i} \mid J_{i}=f\right) \operatorname{Pr}\left(J_{i}=f\right)+y^{\ell} \operatorname{Pr}\left(J_{i}=h\right)\right)-\left(E\left(Y_{h i} \mid J_{i}=h\right) \operatorname{Pr}\left(J_{i}=h\right)+y^{u} \operatorname{Pr}\left(J_{i}=f\right)\right) \\
& \quad \leq A T E \leq \\
& \left(E\left(Y_{f i} \mid J_{i}=f\right) \operatorname{Pr}\left(J_{i}=f\right)+y^{u} \operatorname{Pr}\left(J_{i}=h\right)\right)-\left(E\left(Y_{h i} \mid J_{i}=h\right) \operatorname{Pr}\left(J_{i}=h\right)+y^{\ell} \operatorname{Pr}\left(J_{i}=f\right)\right)
\end{aligned}
$$

In practice the bounds above can yield wide ranges and are often not particularly informative. A number of other assumptions can be used to decrease the size of the identified set.

Manski $(1990,1995)$ shows that one method of tightening the bounds is with an instrumental variable. We can write the expressions (5.20) and (5.21) conditional on $Z_{i}=z$ for any $z \in \operatorname{supp}\left(Z_{i}\right)$ as for each $j \in\{f, h\}$,

$$
\begin{aligned}
E\left(Y_{j i} \mid Z_{i}=z\right) & =E\left(Y_{j i} \mid J_{i}=f, Z_{i}=z\right) \operatorname{Pr}\left(J_{i}=f \mid Z_{i}=z\right) \\
& +E\left(Y_{j i} \mid J_{i}=h, Z_{i}=z\right) \operatorname{Pr}\left(J_{i}=h \mid Z_{i}=z\right)
\end{aligned}
$$

Since $Z_{i}$ is, by assumption, mean independent of $Y_{f i}$ and $Y_{h i}$ (it only affects the probability of choosing one occupation versus the other), then $E\left(Y_{f i} \mid Z_{i}=z\right)=E\left(Y_{f i}\right)$ and $E\left(Y_{h i} \mid Z_{i}=z\right)=E\left(Y_{h i}\right)$. Assume there is a binary instrumental variable, $Z_{i}$, which equals either 0 or 1 . We can then follow exactly the same argument as in equations 
(5.22) and (5.23), but conditioning on $Z_{i}$ and using equation (5.25) yields

$$
\begin{aligned}
& E\left(Y_{f i} \mid J_{i}=f, Z_{i}=1\right) \operatorname{Pr}\left(J_{i}=f \mid Z_{i}=1\right)+y^{\ell} \operatorname{Pr}\left(J_{i}=h \mid Z_{i}=1\right) \\
\leq & E\left(Y_{f i}\right) \leq \\
& \leq E\left(Y_{f i} \mid J_{i}=f, Z_{i}=1\right) \operatorname{Pr}\left(J_{i}=f \mid Z_{i}=1\right)+y^{u} \operatorname{Pr}\left(J_{i}=h \mid Z_{i}=1\right) \\
& E\left(Y_{h i} \mid J_{i}=h, Z_{i}=0\right) \operatorname{Pr}\left(J_{i}=h \mid Z_{i}=0\right)+y^{\ell} \operatorname{Pr}\left(J_{i}=f \mid Z_{i}=0\right) \\
\leq & E\left(Y_{h i}\right) \leq \\
& E\left(Y_{h i} \mid J_{i}=h, Z_{i}=0\right) \operatorname{Pr}\left(J_{i}=h \mid Z_{i}=0\right)+y^{u} \operatorname{Pr}\left(J_{i}=f \mid Z_{i}=0\right) .
\end{aligned}
$$

Thus we can bound $A T E=E\left(Y_{f i}\right)-E\left(Y_{h i}\right)$ from below by subtracting (5.27) from $(5.26)$ :

$$
\begin{aligned}
& E\left(Y_{f i} \mid J_{i}=f, Z_{i}=1\right) \operatorname{Pr}\left(J_{i}=f \mid Z_{i}=1\right)+y^{\ell} \operatorname{Pr}\left(J_{i}=h \mid Z_{i}=1\right) \\
& \quad-E\left(Y_{h i} \mid J_{i}=h, Z_{i}=0\right) \operatorname{Pr}\left(J_{i}=h \mid Z_{i}=0\right)+y^{u} \operatorname{Pr}\left(J_{i}=f \mid Z_{i}=0\right) \\
& \quad \leq A T E \leq \\
& E\left(Y_{f i} \mid J_{i}=f, Z_{i}=1\right) \operatorname{Pr}\left(J_{i}=f \mid Z_{i}=1\right)+y^{u} \operatorname{Pr}\left(J_{i}=h \mid Z_{i}=1\right) \\
& \quad-E\left(Y_{h i} \mid J_{i}=h, Z=0\right) \operatorname{Pr}\left(J_{i}=h \mid Z_{i}=0\right)+y^{\ell} \operatorname{Pr}\left(J_{i}=f \mid Z_{i}=0\right) .
\end{aligned}
$$

Our choice of a binary value of $Z_{i}$ can be trivially relaxed. In the cases in which $Z_{i}$ takes on many values one could choose any two values in the support of $Z_{i}$ to get upper and lower bounds. If our goal is to minimize the size of the set we would choose the values $z^{\ell}$ and $z^{h}$ to minimize the difference between the upper and lower bounds in (5.28):

$$
\left(y^{u}-y^{\ell}\right)\left[\operatorname{Pr}\left(J_{i}=h \mid Z_{i}=z^{h}\right)+\operatorname{Pr}\left(J_{i}=f \mid Z_{i}=z^{\ell}\right)\right]
$$

The importance of support conditions once again becomes apparent from this expression. If we could find values $z^{\ell}$ and $z^{h}$ such that

$$
\begin{aligned}
& \operatorname{Pr}\left(J_{i}=h \mid Z_{i}=z^{h}\right)=0 \\
& \operatorname{Pr}\left(J_{i}=f \mid Z_{i}=z^{\ell}\right)=0
\end{aligned}
$$

then this expression is zero and we obtain point identification of the ATE. When $\operatorname{Pr}\left(J_{i}=h \mid Z_{i}=z\right)$ or $\operatorname{Pr}\left(J_{i}=f \mid Z_{i}=z\right)$ are bounded from below we are only 
able to obtain set estimates. A nice aspect of this is that it represents a nice middle point between identifying LATE versus claiming the ATE is not identified. If the identification at infinity effect is not exactly true, but approximately true so that one can find values of $z^{\ell}$ and $z^{h}$ so that $\operatorname{Pr}\left(J_{i}=h \mid Z_{i}=z^{h}\right)$ and $\operatorname{Pr}\left(J_{i}=f \mid Z_{i}=z^{\ell}\right)$ are small, then the bounds will be tight. If one cannot find such values, the bounds will be far apart.

In many cases these bounds may be wide. Wide bounds can be viewed in two ways. One interpretation is that the bounding procedure is not particularly helpful in learning about the true ATE. However, a different interpretation is that it shows that the data, without additional assumptions, is not particularly helpful for learning about the ATE. Below we discuss additional assumptions for tightening the bounds on the ATE, such as Monotone treatment response, Monotone treatment selection, Monotone instruments. In order to keep matters simple, below we assume that there is no exclusion restriction. However, if a exclusion restriction is known, this allows us to tighten the bounds.

Next we consider the assumption of Monotone Treatment Response introduced in Manski (1997) which we write as

\section{Assumption 5.4. Monotone Treatment Response}

$$
Y_{f i} \geq Y_{h i}
$$

with probability one.

In the fishing/hunting example this is not a particularly natural assumption, but for many applications in labor economics it is. Suppose we are interested in knowing the returns to a college degree, and $Y_{f i}$ is income for individual $i$ if a college graduate whereas $Y_{h i}$ is income if a high school graduate. It is reasonable to believe that the causal effect of school or training cannot be negative. That is, one could reasonably assume that receiving more education can't causally lower your wage. Thus, Monotone Treatment Response seems like a reasonable assumption in this case. This can lower the bounds above quite a bit because now we know that

$$
\begin{aligned}
& E\left(Y_{f i} \mid J_{i}=h\right) \geq E\left(Y_{h i} \mid J_{i}=h\right) \\
& E\left(Y_{h i} \mid J_{i}=f\right) \leq E\left(Y_{f i} \mid J_{i}=f\right) .
\end{aligned}
$$

From this Manski (1997) shows that

$$
0 \leq A T E
$$


Another interesting assumption that can also help tighten the bounds is the Monotone Treatment Selection assumption introduced in Manski and Pepper (2000). In our framework this can be written as

Assumption 5.5. Monotone Treatment Selection: for $j=f$ or $h$,

$$
E\left(Y_{j i} \mid J_{i}=f\right) \geq E\left(Y_{j i} \mid J_{i}=h\right)
$$

Again this might not be completely natural for the fishing/hunting example, but may be plausible in many other cases. For example it seems like a reasonable assumption in schooling if we believe that there is positive sorting into schooling. Put differently, suppose the average college graduate is a more able person than the average high school graduate and would earn higher income, even if she did not have the college degree. If this is true, then the average difference in earnings between college and high school graduates overstates the true causal effect of college on earnings. This also helps to further tighten the bounds as this implies that

$$
A T E \leq E\left(Y_{f i} \mid J_{i}=f\right)-E\left(Y_{h i} \mid J_{i}=h\right)
$$

Note that by combining the MTR and MTS assumption, one can get the tighter bounds:

$$
0 \leq A T E \leq E\left(Y_{f i} \mid J_{i}=f\right)-E\left(Y_{h i} \mid J=h\right)
$$

Manski and Pepper (2000) also develop the idea of a monotone instrumental variable. An instrumental variable is defined as one for which for any two values of the instrument $z_{a}$ and $z_{b}$,

$$
E\left(Y_{j i} \mid Z_{i}=z_{a}\right)=E\left(Y_{j i} \mid Z_{i}=z_{b}\right)
$$

In words, the assumption is that the instrument does not directly affect the outcome variable $Y_{j i}$. It only affects one's choices. Using somewhat different notation, but their exact wording they define a monotone instrumental variable in the following way

Assumption 5.6. Let $\mathcal{Z}$ be an ordered set. Covariate $Z_{i}$ is a monotone instrumental variable in the sense of mean-monotonicity if, for $j \in\{f, h\}$, each value of $x$, and all $\left(z_{b}, z_{a}\right) \in(\mathcal{Z} \times \mathcal{Z})$ such that $z_{b} \geq z_{a}$,

$$
E\left(Y_{j i} \mid X_{i}=x, Z_{i}=z_{b}\right) \geq E\left(Y_{j i} \mid X_{i}=x, Z_{i}=z_{a}\right)
$$


This is a straight generalization of the instrumental variable assumption, but imposes much weaker requirements for an instrument. It does not require that the instrument be uncorrelated with the outcome, but simply that the outcome monotonically increase with the instrument. An example is that parental income has often been used as an instrument for education. Richer parents are better able to afford a college degree for their child. However, it seems likely that the children of rich parents would have had high earnings, even in the absence of a college degree.

They show that this implies that

$$
\begin{aligned}
\sum_{z \in \mathcal{Z}} \operatorname{Pr}\left(Z_{i}=z\right)\left\{\sup _{z_{a} \leq z}\left[E\left(Y_{i} \mid Z_{i}=z_{a}, J_{i}=f\right) \operatorname{Pr}\left(J_{i}=f \mid Z_{i}=z_{a}\right)+y^{\ell} \operatorname{Pr}\left(J_{i}=h \mid Z_{i}=z_{a}\right)\right]\right\} & \left\{\inf _{z_{b} \geq z}\left[E\left(Y_{i} \mid Z_{i}=z_{b}, J_{i}=h\right) \operatorname{Pr}\left(J_{i}=h \mid Z_{i}=z_{b}\right)+y^{u} \operatorname{Pr}\left(J_{i}=f \mid Z_{i}=z_{b}\right)\right]\right\} \\
-\sum_{z \in \mathcal{Z}} \operatorname{Pr}\left(Z_{i}=z T E \leq\right. & \\
& \leq \sum_{z \in \mathcal{Z}} \operatorname{Pr}\left(Z_{i}=z\right)\left\{\inf _{z_{b} \geq z}\left[E\left(Y_{i} \mid Z_{i}=z_{b}, J_{i}=f\right) \operatorname{Pr}\left(J_{i}=f \mid Z_{i}=z_{b}\right)+y^{u} \operatorname{Pr}\left(J_{i}=h \mid Z_{i}=z_{b}\right)\right]\right\} \\
- & \sum_{z \in \mathcal{Z}} \operatorname{Pr}\left(Z_{i}=z\right)\left\{\sup _{z_{a} \leq z}\left[E\left(Y_{i} \mid Z_{i}=z_{a}, J_{i}=h\right) \operatorname{Pr}\left(J_{i}=h \mid Z_{i}=z_{a}\right)+y^{\ell} \operatorname{Pr}\left(J_{i}=f \mid Z_{i}=z_{a}\right)\right]\right\}
\end{aligned}
$$

One can obtain tighter bounds by combining the Monotone Instrumental Variable assumption with the Monotone Treatment Response assumption but we do not explicitly present this result.

Blundell et al. (2007) estimate changes in the distribution of wages in the United Kingdom using bounds to allow for the impact of non-random selection into work. They first document the growth in wage inequality among workers over the 1980s and 1990s. However, they point out that rates of non-participation in the labor force have grown on the UK over the same time period. Nevertheless, they show that selection effects alone cannot explain the rise in inequality observed among workers: the worst case bounds establish that inequality has increased. However, worst case bounds are not sufficiently informative to understand such questions as whether most of the rise in wage inequality is due to increases in wage inequality within education groups versus across education groups. Next, they add in additional assumptions to tighten the bounds. First, they assume the probability of work is higher for those with higher wages, which is essentially the Monotone Treatment Selection assumption shown in Assumption 5.5. Second, they make the Monotone Instrumental Variables assumption 
shown in Assumption 5.6. They assume that higher values out of work benefit income are positively associated with wages. They show that both of these assumptions tighten the bounds considerably. They find that when these additional restrictions are made, then they can show that both within group and between group inequality has increased.

\subsection{Using Selection on Observables to Infer Selection on Un- observables}

Altonji, Elder, and Taber (2005a) suggest another approach which is to use the amount of selection on observable covariates as a guide to the potential amount of selection on unobservables. To motivate this approach, consider an experiment in which treatment status is randomly assigned. The key to random assignment is that it imposes that treatment status be independent of the unobservables in the treatment model. Since they are unobservable, one can never explicitly test whether the treatment was truly random. However, if randomization was carried out correctly, treatment should also be uncorrelated with observable covariates. This is testable and applying this test is standard in experimental approaches.

Researchers use this same argument in non-experimental cases as well. If a researcher wants to argue that his instrument or treatment is approximately randomly assigned, then it should be uncorrelated with observable covariates as well. Even if this is strictly not required for consistent estimates of instrumental variables, readers may be skeptical of the assumption that the instrument is uncorrelated with the unobservables if it is correlated with the observables. Researchers often test for this type of relationship as well. ${ }^{17}$ The problem with this approach is that simply testing the null of uncorrelatedness is not that useful. Just because you reject the null does not mean it isn't approximately true. We would not want to throw out an instrument with a tiny bias just because we have a data set large enough to detect a small correlation between it and an observable. Along the same lines, just because you fail to reject the null does not mean it is true. If one has a small data set with little power one could fail to reject the null even though the instrument is poor. To address these issues, Altonji, Elder, and Taber (2005a) design a framework that allows them to describe how large the treatment effect would be if "selection on the unobservables is the same as selection

\footnotetext{
${ }^{17}$ Altonji, Elder, and Taber (2005a) discuss a number of studies that do so.
} 
on the observables."

Their key variables are discrete, so they consider a latent variable model in which a dummy variable for graduation from high school can be written as

$$
G_{i}= \begin{cases}1 & Y_{i}^{*} \geq 0 \\ 0 & Y_{i}^{*}<0\end{cases}
$$

where $Y_{i}^{*}$ can be written as

$$
\begin{aligned}
Y_{i}^{*} & =\beta_{0}+\alpha D_{f i}+\sum_{j=1}^{K} W_{i j} \beta_{j} \\
& =\beta_{0}+\alpha D_{f i}+\sum_{j=1}^{K} S_{j} W_{i j} \beta_{j}+\sum_{j=1}^{K}\left(1-S_{j}\right) W_{i j} \beta_{j} \\
& =\beta_{0}+\alpha D_{f i}+X_{i}^{\prime} \beta+\nu_{i} .
\end{aligned}
$$

$W_{i j}$ represent all covariates, both those that are observable to the econometrician and those that are unobservable, the variable $S_{j}$ is a dummy variable representing whether the covariate is observable to the empirical researcher, $X_{i}^{\prime} \beta=\sum_{j=1}^{K} S_{j} W_{i j} \beta_{j}$ represents the observable part of the index, and $\nu_{i}=\sum_{j=1}^{K}\left(1-S_{j}\right) W_{i j} \beta_{j}$ denotes the unobservable part.

Within this framework, one can see that different assumptions about what dictates which observables are chosen $\left(S_{j}\right)$ can be used to identify the model. Their specific goal is to quantify what it means for "selection on the observables to be the same as selection on the unobservables." They argue that the most natural way to formalize this idea is to assume that $S_{j}$ is randomly assigned so that the unobservables and observables are drawn from the same underlying distribution.

The next question is what this assumption implies on the data that can be useful for identification. They consider the projection:

$$
\operatorname{proj}\left(Z_{i} \mid X_{i}^{\prime} \beta, \nu_{i}\right)=\phi_{0}+\phi X_{i}^{\prime} \beta+\phi_{\varepsilon} \nu_{i}
$$

where $Z_{i}$ can be any random variable. They show that if $S_{j}$ is randomly assigned,

$$
\phi \approx \phi_{\varepsilon}
$$

This restriction is typically sufficient to insure identification of $\alpha{ }^{18}$

\footnotetext{
${ }^{18}$ In some cases it is not point identification, but either 2 or 3 different points.
} 
Altonji, Elder and Taber (2005) argue that for their example this is an extreme assumptions and the truth is somewhere in between this assumption and the assumption that $Z_{i}$ is uncorrelated with the unobservables which would correspond to $\phi_{\varepsilon}=0$. They assume that when $\phi>0$,

$$
0 \leq \phi_{\varepsilon} \leq \phi
$$

There are at least three arguments for why selection on unobservables would be expected to be less severe than selection on observables (as it is measured here). First, some of the variation in the unobservable is likely just measurement in the dependent variable. Second, data collectors likely collect the variables that are likely to be correlated with many things. Third, there is often a time lapse between the time the baseline data is collected (the observables) and when the outcome is realized. If unanticipated events occur in between these two time periods, that would lead to the result.

Notice that if $\phi=0$ then assuming $\phi_{\varepsilon}=\phi$ is the same as assuming $\phi_{\varepsilon}=0$. However, if $\phi$ were very large the two estimates would be very different which would shed doubt on the assumption of random assignment. Since $\phi$ essentially picks up the relationship between the instrument and the observable covariates, the bounds would be wide when there is a lot of selection on observables and will be tight when there is little selection on observables.

Altonji, Elder, and Taber consider the case of whether the decision to attend Catholic high school affects outcomes such as test scores and high school graduation rates. Those who attend Catholic schools have higher graduation rates than those who do not attend Catholic schools. However, those who attend Catholic may be very different than those who do not. They find that (on the basis of observables) while this is true in the population, it is not true when one conditions on the individuals who attend Catholic school in eighth grade. To formalize this, they use their approach and estimate the model under the two different assumptions. In their application the projection variable, $Z_{i}$, is the latent variable determining whether an individual attends Catholic school. First they estimate a simple probit of high school graduation on Catholic high school attendance as well as many other covariates. This corresponds to the $\phi_{\varepsilon}=0$ case. They find a marginal effect of 0.08 meaning that Catholic school raises high school graduation by eight percentage points. Next they estimate a bivariate probit of Catholic high school attendance and high school graduation subject to the 
constraint that $\phi_{\varepsilon}=\phi$. In this case they find a Catholic high school effect of 0.05. The closeness of these two estimates strongly suggests that the Catholic high school effect is not simply a product omitted variable bias. The tightness of the two estimates arose both because $\phi$ was small and because they use a wide array of powerful explanatory variables.

\section{Duration Models and Search Models}

In this section we relate the previous discussion to the competing risks model and the search model. We show that the competing risk model can be written in a way that is almost identical to the Roy model. We also show how the basic ideas of exclusion restrictions can be used to identify a version of a search model.

\subsection{Competing Risks Model}

With duration data a researcher observes the elapsed time until some event occurs. The prototypical example in labor economics is the duration of unemployment and we focus on that example. We explain why identification of this model is almost identical to identification of the Roy model. Let $T_{i}$ denote the length of an unemployment spell. There are (at least) four different ways to characterize the distribution of $T_{i}$. The first is the cumulative distribution function $F(t) \equiv \operatorname{Pr}\left(t>T_{i}\right)$, which in the context of unemployment durations is the probability the individual found a job. The second is the density function $f$. The third is the survivor function defined as

$$
S(t) \equiv \operatorname{Pr}\left(T_{i}>t\right)=1-F(t)
$$

The fourth is the hazard function, which is the job finding rate at time $t$, given that the individual was unemployed at time $t$ :

$$
\begin{aligned}
h(t) & \equiv \lim _{\delta \rightarrow 0} \frac{\operatorname{Pr}\left(T_{i} \leq t+\delta \mid T_{i} \geq t\right)}{\delta} \\
& =\frac{f(t)}{S(t)}
\end{aligned}
$$


The link between the hazard rate and survivor function is:

$$
\begin{aligned}
h(t)=\frac{f(t)}{S(t)} & =\frac{d F(t) / d t}{S(t)} \\
& =\frac{-d S(t) / d t}{S(t)} \\
& =\frac{-d \log S(t)}{d t} .
\end{aligned}
$$

There is a large literature on identification of duration models. Heckman and Taber (1994), Van den Berg (2001), and Abbring (2010) provide excellent surveys of this literature. ${ }^{19}$ Rather than survey the full literature here we relate it to our previous discussion. Given that $T_{i}$ must be positive, it is natural to model $T_{i}$ using the basic framework we have been using all along:

$$
\log \left(T_{i}\right)=g\left(X_{i}\right)+\varepsilon_{i}
$$

Clearly if we could observe the distribution of $\log \left(T_{i}\right)$ conditional on $X_{i}$, identification of $g$ and the distribution of $\varepsilon_{i}$ would be straightforward.

However, often we cannot observe the full duration of $T_{i}$ because the spell (or our observation of it) is truncated before the worker is re-employed. For example, the worker may die, be lost from the data, or the survey may end. In the classic medical example we might want to estimate the duration until a patient has a heart attack, but if she dies from cancer we never observe this event. Hence the name "competing risk model." To put this in the context of our Roy model example, suppose an unemployed worker would take the first offer they received and they can get an offer as a fisherman or a hunter. Define the model as

$$
\begin{aligned}
& \log \left(T_{f i}\right)=g_{f}\left(X_{i}\right)+\varepsilon_{f i} \\
& \log \left(T_{h i}\right)=g_{h}\left(X_{i}\right)+\varepsilon_{h i}
\end{aligned}
$$

where $T_{f i}$ and $T_{h i}$ are the amount of time it would take until the worker received an offer as a fisherman or as a hunter, $X_{i}$ denotes observable variables that are independent of the unobservables $\left(\varepsilon_{f i}, \varepsilon_{h i}\right){ }^{20}$ The econometrician can observe whether the worker

\footnotetext{
${ }^{19}$ Key papers include Elbers and Ridder (1982), Heckman and Singer (1984a,b), Ridder (1990), Honoré (1993), and Abbring and Ridder (2009).

${ }^{20} \mathrm{We}$ do not need to make use of exclusion restrictions here so we do not distinguish between observables that may enter differently.
} 
becomes a fisherman or a hunter and the length of the unemployment spell. However, notice that as Heckman and Honoré (1990) point out, this is just another version of the Roy model. Rather than observe the maximum of $Y_{f i}$ and $Y_{h i}$, the econometrician observes the minimum of $\log \left(T_{f i}\right)$ and $\log \left(T_{h i}\right)$.

The specification (6.2) and (6.3) above is not the way that many researchers choose to model duration data. Often they model the hazard function directly as it is sometimes easier to interpret. Moreover, if the observable covariates change over time, the hazard model is a more reasonable way to model the durations. The most common specification is the mixed proportional hazard model

$$
h\left(t \mid X_{i}=x\right)=\xi(t) \phi(x) \omega_{i}
$$

where $\xi(t)$ is referred to as the baseline hazard, $\omega_{i}$ is an unobservable variable which is independent of the observables, and $X_{i}$ denotes observable characteristics. Most studies find that the hazard rate for finding a job tends to decline with the unemployment duration. The model above allows for two possible interpretations of this empirical regularity. First, it could be that as unemployment durations lengthen, skills depreciate, making it harder to find a job. This is captured by $\xi(t)$. Second, it could be that some people are just less able to find a job than others in ways not captured by observables. This is captured in $\omega_{i}$. Van den Berg (1999) provides a thorough discussion of this model.

Heckman and Honoré (1989) show how to map the hazard specification into a framework that is similar to what we use in our analysis of the Roy model. The transformation is simplest is when $\xi(t)=1$. In that case one can write the survivor function as

$$
\operatorname{Pr}\left(T_{i}>t \mid X_{i}=x, \omega_{i}=\omega\right)=e^{-t \phi(x) \omega} .
$$

It is straightforward to derive equation (6.4) using the survivor function (6.5) and equation (6.1). Define $g(\cdot)=-\log (\phi(\cdot))$ and $F_{\omega}$ to be the distribution of $\omega_{i}$. In order to obtain the cumulative density function of unemployment durations we must 
integrate over the distribution of unemployed individuals:

$$
\begin{aligned}
\operatorname{Pr}\left(T_{i} \leq t \mid X_{i}=x\right) & =\int 1-e^{-t \phi(x) \omega_{i}} d F_{\omega} \\
& =\int 1-\exp \left(-\exp \left(\log (t)-g(x)+\log \left(\omega_{i}\right)\right)\right) d F_{\omega} \\
& \equiv F_{\widetilde{\omega}}(\log (t)-g(x))
\end{aligned}
$$

where $F_{\widetilde{\omega}}$ is defined implicitly by this relationship. Note that $F_{\widetilde{\omega}}$ is a legitimate CDF, as it is strictly increasing from 0 to $1 .{ }^{21}$ Thus one can think of the data generating process as

$$
\log \left(T_{i}\right)=g\left(X_{i}\right)+\widetilde{\omega}_{i}
$$

where $\widetilde{\omega}_{i}$ is distributed according to $F_{\widetilde{\omega}}$ and is independent of $X_{i}$.

In the more general case in which $\xi(t)$ is not constant, it is well known that one can write the survivor function as

$$
e^{-\Xi(t) \phi\left(X_{i}\right) \omega_{i}}
$$

where $\Xi$ is the integrated hazard

$$
\Xi(t) \equiv \int_{0}^{t} \xi(t) d t .
$$

Equation (6.7) differs from equation (6.5) by the term $\Xi(t)$ instead of $t$. Thus replacing $t$ with $\Xi(t)$ in equation (6.6) yields

$$
\log \left(\Xi\left(T_{i}\right)\right)=g\left(X_{i}\right)+\widetilde{\omega}_{i}
$$

Heckman and Honoré (1989) use a more general framework to think about the competing risks model in which the probability of not getting a fishing job by time $t_{f}$ and not getting a hunting job by time $t_{h}, S\left(t_{f}, t_{h} \mid X_{i}=x\right)$, can be written as

$$
S\left(t_{f}, t_{h} \mid X_{i}=x\right)=K\left(\exp \left\{-\Xi_{f}\left(t_{f}\right) \phi_{f}(x)\right\}, \exp \left\{-\Xi_{h}\left(t_{h}\right) \phi_{h}(x)\right\}\right)
$$

where $\phi_{j}(x)=\exp \left(-g_{j}(x)\right)$ for $j=f, h$. This is a generalization of a model in which

$$
\begin{aligned}
& \log \left(\Xi_{f}\left(T_{f i}\right)\right)=g_{f}\left(X_{i}\right)+\widetilde{\omega}_{f i} \\
& \log \left(\Xi_{h}\left(T_{h i}\right)\right)=g_{h}\left(X_{i}\right)+\widetilde{\omega}_{h i}
\end{aligned}
$$

\footnotetext{
${ }^{21}$ It is the distribution of a convolution between $\log \left(\omega_{i}\right)$ and an extreme value.
} 
because

$$
\begin{aligned}
& S\left(t_{f}, t_{h} \mid X_{i}=x\right)=\operatorname{Pr}\left[\log \left(\Xi_{f}\left(T_{f i}\right)\right)>\log \left(\Xi_{f}\left(t_{f}\right)\right), \log \left(\Xi_{h}\left(T_{h i}\right)\right)>\log \left(\Xi_{h}\left(t_{h}\right)\right) \mid X_{i}=x\right] \\
& =\operatorname{Pr}\left[g_{f}(x)+\widetilde{\omega}_{f i}>\log \left(\Xi_{f}\left(t_{f}\right)\right), g_{h}(x)+\widetilde{\omega}_{h i}>\log \left(\Xi_{h}\left(t_{h}\right)\right)\right] \\
& =\operatorname{Pr}\left[-\widetilde{\omega}_{f i}<-\log \left(\Xi_{f}\left(t_{f}\right)\right)+g_{f}(x),-\widetilde{\omega}_{h i}<-\log \left(\Xi_{h}\left(t_{h}\right)\right)+g_{h}(x)\right] \\
& =F_{-\widetilde{\omega}_{f i}-\widetilde{\omega}_{h i}}\left(-\log \left(\Xi_{f}\left(t_{f}\right)\right)+g_{f}(x),-\log \left(\Xi_{h}\left(t_{h}\right)\right)+g_{h}(x)\right) \\
& \equiv K\left(\exp \left\{-\Xi_{f}\left(t_{f}\right) \phi_{f}(x)\right\}, \exp \left\{-\Xi_{h}\left(t_{h}\right) \phi_{h}(x)\right\}\right)
\end{aligned}
$$

where $F_{-\widetilde{\omega}_{f i}-\widetilde{\omega}_{h i}}$ is the joint $\operatorname{CDF}$ of $\left(-\widetilde{\omega}_{f i}^{*},-\widetilde{\omega}_{h i}^{*}\right)$, and $K$ is defined implicitly as $K(a, b)=F_{-\widetilde{\omega}_{f i}-\widetilde{\omega}_{h i}}(-\log (-\log (a)),-\log (-\log (b)))$.

Heckman and Honoré (1989), Theorem 1 contains the following result. We reproduce their result, only altering the notation.

Theorem 6.1. Assume that $\left(T_{f i}, T_{h i}\right)$ has the joint survivor function as given in (6.8). Then $\Xi_{f}, \Xi_{h}, \phi_{f}, \phi_{h}$, and $K$ are identified from the identified minimum of $\left(T_{f i}, T_{h i}\right)$ under the following assumptions

1. $K$ is continuously differentiable with partial derivatives $K_{1}$ and $K_{2}$ for $i=1,2$ the limit as $n \rightarrow \infty$ of $K_{i}\left(\eta_{1 n}, \eta_{2 n}\right)$ is finite for all sequences of $\eta_{1 n}, \eta_{2 n}$ for which $\eta_{1 n} \rightarrow 1$ and $\eta_{2 n} \rightarrow 1$ for $n \rightarrow \infty$. We also assume that $K$ is strictly increasing in each of its arguments in all of $[0,1] \times[0,1]$.

2. $\Xi_{f}(1)=1, \Xi_{h}(1)=1, \phi_{f}\left(x_{0}\right)=1$ and $\phi_{h}\left(x_{0}\right)=1$ for some fixed point $x_{0}$ in the support $X$.

3. The support of $\left\{\phi_{f}(x), \phi_{h}(x)\right\}$ is $(0, \infty) \times(0, \infty)$.

4. $\Xi_{f}$ and $\Xi_{h}$ are nonnegative, differentiable, strictly increasing functions, except that we allow them to be $\infty$ for finite $t$.

(Proof in Heckman and Honoré, 1989 )

Since the model is almost identical to the Roy model, the intuition for identification is very similar so we don't review it here. We do mention a few things about these assumptions. First note that assumption (2) in Theorem 6.1 is just a normalization as one cannot separate the scales of $\phi_{f}, \Xi_{f}$, and $\nu_{f}$. The more notable difference between this and the theorem we presented in the Roy model section above is the lack 
of exclusion restrictions. What is crucial in being able to do this is the assumptions about $K$ in assumption (1). In their proof they show that for any $x$ in the support of $X_{i}$

$$
\lim _{t \rightarrow 0} \frac{\frac{\partial P r\left(T_{f i}<t, T_{h i}>T_{f i} \mid X_{i}=x\right)}{\partial t}}{\frac{\partial \operatorname{Pr}\left(T_{f i}<t, T_{h i}>T_{f i} \mid X_{i}=x_{0}\right)}{\partial t}}=\phi_{f}(x) .
$$

One could in principle use this form of identification for the Roy model, but it is somewhat less natural in the Roy framework as taking the limit as $t \rightarrow 0$ corresponds to taking limits as the log of wages become arbitrarily large. It also makes heavy use of the independence assumption which is not necessary for identification of $g_{f}$ when one has exclusion restrictions. Finally, the basic approach will not expand to the "labor supply" model in which we only observe wages in one sector and to the generalized Roy model in the same way that exclusion restrictions do.

Abbring and van den Berg (2003) extends Heckman and Honoré's (1989) results on the mixed proportional hazards competing risk models in a few ways including generalizing the assumptions for identification somewhat and considering identification in the case in which researchers observe multiple spells.

\subsection{Search Models}

Eckstein and van den Berg (2007) present a nice survey of Empirical Search models. We avoid a general discussion, but rather combine the proportional hazard model with a search model. In a well known result Flinn and Heckman (1982) show that the search model is not fully identified. They use the Lippman and McCall (1976) search model in which workers search for jobs until their wage exceeds their reservation wage. In this model, one essentially assumes that the worker stays at the job forever. All workers are assumed to be ex-ante identical and face the same distribution of offered wages which we denote by $F$. The reservation wage $w^{r}$ is the point at which the individual is indifferent between taking the job and continued search. It is defined implicitly by the formula

$$
c+w^{r}=\frac{\lambda}{r} \int_{w^{r}}^{\infty}\left(x-w^{r}\right) d F(x)
$$

where $c$ is search cost, $r$ is the interest rate, and $\lambda$ is the hazard rate of finding a job.

Flinn and Heckman (1982) assume that one observes the time until finding a job $\left(T_{i}\right)$ and the wage a worker receives conditional on finding the job. The only source of 
heterogeneity in the model comes from the timing of the job offers and the draw from the wage offer distribution. Clearly one can identify the distribution of accepted wage offers which is the distribution of observed wages. The reservation wage is the lowest acceptable wage, so a one can identify $w^{r}$ as the minimum observed wage. Then they can identify

$$
\frac{f(x)}{1-F\left(w^{r}\right)} \text { for } x \geq w^{r} \text {. }
$$

They can also identify the hazard rates of job finding which is

$$
\lambda\left(1-F\left(w^{r}\right)\right) .
$$

However, this is all that can be identified. In particular, one cannot separate $\lambda$ from $\left(1-F\left(w^{r}\right)\right)$. Furthermore, the distribution of wage offers below the reservation wage is not identified. This is quite intuitive. Since nobody works at a salary below the reservation wage, we do not have any information from the data on what that distribution might look like. ${ }^{22}$ Furthermore, identification of the model above relies on the strong assumption that people are identical. All dispersion in observed wages comes from identical people with identical skills being offered different wages. It also implies a constant hazard rate of finding jobs $\lambda$, which is at odds with the data.

By using exclusion restrictions and using some of the ideas from the Roy model with the arguments from the mixed proportional hazard model, most of the components of the model can be identified. In particular let the arrival rate of job offers be

$$
\lambda_{i}=\phi\left(X_{\lambda i}, X_{0 i}\right) \omega_{i}
$$

where now $X_{\lambda i}$ is an exclusion restriction that influences the arrival rate, but not any other aspect of the model. We assume that search cost is defined as

$$
\log \left(C_{i}\right)=g_{h}\left(X_{h i}, X_{0 i}\right)+\varepsilon_{h i}
$$

Finally we assume the wage offer that individual $i$ would receive at time $t$ is

$$
\log \left(W_{f i t}\right)=g_{f}\left(X_{f i}, X_{0 i}\right)+\varepsilon_{f i t} .
$$

\footnotetext{
${ }^{22}$ Of course this raises an interesting question. What does it mean for a firm to make an offer that it knows no worker would ever take? In most wage posting models, a firm would never post a wage that no worker would take (see e.g. Burdett and Mortensen, 1998). However, if there is a job match component, one can also write down a model in which one could define the counterfactual wage at which a worker would be paid at a job in which he would never take (whether that offer is actually "extended" or not is largely a semantic issue).
} 
The complicated aspect of this model is that workers may reject the first offer they receive, and then receive a second different offer. Thus we need the time subscript on $\varepsilon_{\text {fit }}$ to denote that this draw can be different. The second issue is that one would expect the distribution of offered $\varepsilon_{f i t}$ to not be identical across workers. We assume that the distribution of $\varepsilon_{f i t}$ is individual specific coming from distribution $F_{i \varepsilon_{f}}$. That is each time a worker gets a new offer it is a draw from the distribution of $F_{i \varepsilon_{f}}$. As above $X_{i}$ is observable and independent of $\left(\nu_{i}, \varepsilon_{f i t}, \varepsilon_{h i}\right)$.

Using the Lippman and McCall (1976) model, define $W_{i}^{*}$ as the solution to the equation

$$
C_{i}+W_{i}^{*}=\frac{\lambda_{i}}{r} \int_{\log \left(W_{i}^{*}\right)-g_{f}\left(X_{f i}, X_{0 i}\right)}^{\infty}\left(e^{g_{f}\left(X_{f i}, X_{0 i}\right)+\varepsilon_{f i t}}-W_{i}^{*}\right) d F_{i \varepsilon_{f}}\left(\varepsilon_{f i t}\right) .
$$

The reservation wage is defined as

$$
W_{i}^{r} \equiv \max \left\{W_{i}^{*}, 0\right\}
$$

If search costs are sufficiently high, $W_{i}^{*}$ could be negative. But because the distribution of wages is bounded below at 0 , the reservation wage would be 0 .

The added assumptions to identify the model are completely analogous to those we used for the Roy model earlier

Assumption 6.1. $\left(\varepsilon_{f i t}, \varepsilon_{h i}, \nu_{i}\right)$ is continuously distributed with support $\mathbb{R}^{3}$, and is independent of $X_{i}$.

Assumption 6.2. $\operatorname{supp}\left(\phi\left(X_{\lambda i}, X_{0 i}\right), g_{f}\left(X_{f i}, x_{0}\right), g_{h}\left(X_{h i}, x_{0}\right)\right)=\mathbb{R}^{+} \times \mathbb{R}^{2}$ for all $x_{0} \in$ $\operatorname{supp}\left(X_{0 i}\right)$.

Assumption 6.3. The marginal distributions of $\varepsilon_{f i t}, \varepsilon_{h i}$, and $\nu_{i}$ have expected values equal to zero. Moreover, the expected value of $e^{\varepsilon_{f i t}}$ is finite.

Assumption 6.4. $X_{i}=\left(X_{f i}, X_{h i}, X_{\lambda i}, X_{0 i}\right)$ can be written as $\left(X_{f i}^{c}, X_{f i}^{d}, X_{h i}^{c}, X_{h i}^{d}, X_{\lambda i}^{c}, X_{\lambda i}^{d}, X_{0 i}^{c}, X_{0 i}^{d}\right)$ where the elements of $X^{c}=\left(X_{f i}^{c}, X_{h i}^{c}, X_{\lambda i}^{c}, X_{0 i}^{c}\right)$ are continuously distributed (no point has positive mass), and $X^{d}=\left(X_{f i}^{d}, X_{f i}^{d}, X_{\lambda i}^{d}, X_{0 i}^{d}\right)$ is distributed discretely (all support points have positive mass).

Assumption 6.5. For any $\left(x_{f}^{d}, x_{h}^{d}, x_{\lambda}^{d}, x_{0}^{d}\right) \in \operatorname{supp}\left(X_{f i}^{d}, X_{h i}^{d}, X_{\lambda i}^{d}, X_{0 i}^{d}\right), g_{f}\left(x_{f}^{c}, x_{f}^{d}, x_{0}^{c}, x_{0}^{d}\right)$, $g_{h}\left(x_{h}^{c}, x_{h}^{d}, x_{0}^{c}, x_{0}^{d}\right)$, and $\phi\left(x_{\lambda}^{c}, x_{\lambda}^{d}, x_{0}^{c}, x_{0}^{d}\right)$ are almost surely continuous across $\left(x^{c}\right) \in \operatorname{supp}\left(X_{i}^{c} \mid\right.$ $\left.X_{i}^{d}=x^{d}\right)$. 
Theorem 6.2. Under assumptions 6.1- 6.5 and that $\phi$ and the distribution of $\omega_{i}$ satisfy the assumptions in Heckman and Honoré (1989), given that we observe $T_{i}$ and $w_{f i T_{i}}$ from the model determined by equations (6.9)-(6.13), we can identify $\phi$ and $g_{f}$ on their support, and $g_{h}$ up to location on a set $\mathcal{X}^{*}$ that has measure 1.

\section{(Proof in Appendix)}

Unlike some of the other models, we have not completely identified the error structure (or the location of $g_{h}$ ). This is probably not surprising given the complexity of $F_{i \varepsilon_{f}}$ and the relatively modest data conditions. ${ }^{23}$

We conclude this section after making three comments. First, it is not clear that one cares about the location of $g_{h}$.

That is, for many interesting policy counterfactuals, identification of the aspects above should be sufficient. Second, with more structure, more features of the model should be identified. ${ }^{24}$ Third, if a researcher observes multiple spells on the same worker, this can add much identifying information. The identification problem arises because if we see one worker making more than another we do not know if it is because the first worker is more productive or if they just happened to get a fortunate draw from offer distribution. With panel data, if we see that the first worker consistently earns more money across many employers, this would suggest that the difference has more to do with ability than with draws from the offer distribution.

We have barely scratched the surface of identification of search models. Many papers being estimated today are based on equilibrium models such as Mortensen and Pissarides (1994), Burdett and Mortensen (1998), or Postel-Vinay and Robin (2002). We think there is much work to be done on identification in these models. ${ }^{25}$

\footnotetext{
${ }^{23}$ Some aspects of the distribution of wages can be identified. For example identification of the marginal distribution of $\omega_{i}$ is straightforward. Describing the distribution of $F_{i \varepsilon_{f}}$ is difficult because it is a distribution of distributions. Given the cost in setting up notation to discuss this, we do not try to characterize this distribution. A typical assumption would be that we could write $\varepsilon_{f i t}=\epsilon_{f i}+\zeta_{f i t}$ where $\epsilon_{f i}$ is an individual specific term that does not vary across wages and $\zeta_{f i t}$ is i.i.d.

${ }^{24}$ Proving identification in nonlinear models such as this one is often quite difficult. This might not be problematic in practice as researchers can search for multiple solutions in the data. If there are multiple solutions, all can be reported. If only one solution exists, this should give a consistent estimate of the truth.

${ }^{25}$ Canals-Cerda (2010) provides a recent example which adds measurement error in wages to the Flinn and Heckman (1982) framework. Barlevy (2008) shows how to non-parametrically identify the wage offer distribution in the presence of measurement error in wages and unobserved heterogeneity in skills.
} 


\section{Forward Looking Dynamic Models}

In this section we discuss an extension of the generalized Roy model into a dynamic framework with uncertainty and forward looking behavior. We show that the basic identification ideas presented above can be generalized to dynamic models. The identification results for the simple models on which we focus can be extended to more complicated environments. We begin with a two period model which in which there are three choices made over two periods. We then discuss some general issues with identifying the components of the Bellman Equation. Finally we present a dynamic Generalized Roy model that one can use for dynamic treatment effect evaluation. Once again, we do not provide a full review of the literature, but focus on expanding the generalized Roy model into a forward looking dynamic model. Abbring (2010) includes a more complete discussion. ${ }^{26}$

\subsection{Two period Discrete Choice Dynamic Model}

We begin with the framework of Taber (2000) who considers a simple version of a dynamic model. To think of this model as an extension of the basic Roy model we go from two occupational choices to three. While we could modify the fishing/hunting example to a dynamic context, it is easiest to think about this in terms of an education model as Taber does. In particular, a student first decides whether to graduate from high school or not. After graduating from high school, she decides whether to attend college or enter the labor market directly. Extending beyond 3 choices is straightforward, but as in Taber we stick to the 3 choice model for expositional purposes. We focus on identification of the choice model and ignore data on earnings until section 7.3 .

First consider the case in which there was no uncertainty or dynamics. We specify the model using the three value functions

$$
\begin{aligned}
V_{c i} & =g_{c}\left(X_{c i}, X_{0 i}\right)+\varepsilon_{c i} \\
V_{d i} & =g_{d}\left(X_{d i}, X_{0 i}\right)+\varepsilon_{d i} \\
V_{h i} & =0
\end{aligned}
$$

\footnotetext{
${ }^{26}$ Recent papers that cover aspects of identification not discussed here include Kasahara and Shimotsu (2009) and Hu and Shum (2009).
} 
where $V_{c i}$ is the value function for a college student, $V_{h i}$ the value function for an individual with exactly a high school degree, and $V_{d i}$ the value function for high school dropout. Individuals choose the option with the highest value function. That is

$$
J_{i}=\operatorname{argmax}\left\{V_{d i}, V_{h i}, V_{c i}\right\} .
$$

If there were no uncertainty in this model it would be a simple polychotomous choice model. Matzkin (1993) considers identification a general class of polychotomous choice modes under a number of different assumptions. One result is that since choices are only identified up to monotonic transformations, $V_{h i}=0$ is a location normalization that we impose at this point. Adding dynamics and uncertainty does not change this result.

Our goal now is to add dynamics and uncertainty to the model. The timing can be seen in the following figure

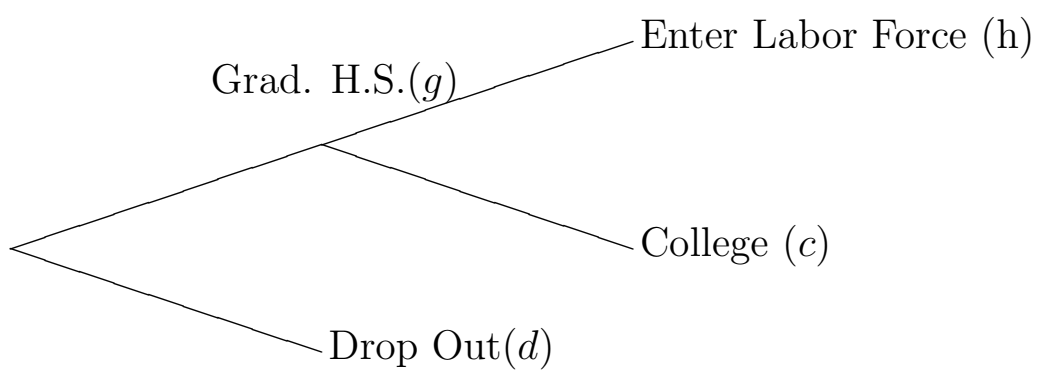

In the first period the agent chooses whether to graduate from high school. If she graduates in the first period, she then chooses whether to go to college in the second. The key aspect of the model is that information will be revealed between the first and second period. The agent's preferences are summarized by lifetime reward function $V_{j i}$ at each terminal state $j \in\{c, h, d\}$. Taber defines $V_{d i}$ so that it is known at the time the high school graduation choice is made. Then in period two, $V_{c i}$ and $V_{h i}$ are known when the choice between $c$ and $h$ is made. That is, in period one the agent does not know $X_{c i}$ or $\varepsilon_{c i}$. The first period information is assumed to be contained in $\left(X_{0 i}, X_{1 i}, \varepsilon_{1 i}\right)$ where $X_{1 i}$ is observable in period one and will be informative about $X_{c i}$ while $\varepsilon_{1 i}$ is unobservable and informative about $\varepsilon_{c i}$. We assume that decisions are made in order to maximize expected lifetime reward. Thus the reward function at node $g$ in 
the first period takes the value

$$
V_{g}\left(x_{1}, x_{d}, x_{0}, \epsilon_{1}\right) \equiv E\left[\max \left\{V_{c i}, V_{h i}\right\} \mid\left(X_{1 i}, X_{d i}, X_{0 i}\right)=\left(x_{1}, x_{d}, x_{0}\right), \varepsilon_{1 i}=\epsilon_{1}\right]
$$

The agent chooses node $d$ if $V_{d i}>V_{g}\left(X_{1 i}, X_{d i}, X_{0 i}, \varepsilon_{1 i}\right)$ and chooses node $g$ otherwise. If she chooses $g$ in the first period she chooses node $c$ in the second if $V_{c i}>V_{h i}$ and node $h$ otherwise.

We let $G\left(X_{c i} \mid\left(X_{1 i}, X_{d i}, X_{0 i}\right)=\left(x_{1}, x_{d}, x_{0}\right)\right)$ denote the distribution of $X_{c i}$ conditional on $\left(X_{1 i}, X_{d i}, X_{0 i}\right)=\left(x_{1}, x_{d}, x_{0}\right)$. We can summarize the information structure as follows

\begin{tabular}{|l|l|l|}
\hline Known to the Agent & $\begin{array}{l}\text { Learned by the Agent } \\
\text { at time one }\end{array}$ & $\begin{array}{l}\text { Observed by } \\
\text { the Econometrician }\end{array}$ \\
\hline$\varepsilon_{1 i}, \varepsilon_{d i}$ & $\varepsilon_{c i}$ & $X_{0 i}, X_{1 i}, X_{d i}$ \\
$X_{0 i}, X_{1 i}, X_{d i}$ & $X_{c i}$ & $X_{c i}$ \\
$G\left(X_{c i} \mid\left(X_{1 i}, X_{d i}, X_{0 i}\right)=\left(x_{1}, x_{d}, x_{0}\right)\right)$ & & $J_{i}$ \\
\hline
\end{tabular}

We first consider identification of $g_{c}$ and $g_{d}$ up to monotonic transformations. We follow Taber (2000) closely except that we use our notation and use stronger assumptions than he does to avoid adding more notation. ${ }^{27}$

Assumption 7.1. For any $\left(x_{c}, x_{0}\right) \in \operatorname{supp}\left\{X_{c i}, X_{0 i}\right\}$,

$$
\begin{aligned}
& \operatorname{supp}\left\{\varepsilon_{d i}\right\}=\mathbb{R}=\operatorname{supp}\left\{g_{d}\left(X_{d i}, x_{0}\right) \mid\left(X_{c i}, X_{0 i}\right)=\left(x_{c}, x_{0}\right)\right\} \\
& \operatorname{supp}\left\{\varepsilon_{c i}\right\}=\mathbb{R}
\end{aligned}
$$

This assumption is analogous to what we have been assuming all along. In order to estimate the full model, we need full support of $g_{d}$ conditional on $\left(X_{c i}, X_{0 i}\right)$.

Assumption 7.2. For any $\left(x_{d}, x_{0}\right) \in \operatorname{supp}\left\{X_{d i}, X_{0 i}\right\}, y \in \mathbb{R}$, and $a \in(0,1)$, there exists a set $\mathcal{X}_{1}\left(x_{f}, x_{0}, y, a\right)$ with positive measure such that for $x_{1} \in \mathcal{X}_{1}\left(x_{f}, x_{0}, y, a\right)$,

(a) $\operatorname{Pr}\left(g_{c}\left(X_{c i}, x_{0}\right)<y \mid\left(X_{1 i}, X_{d i}, X_{0 i}\right)=\left(x_{1}, x_{d}, x_{0}\right)\right)>a$

${ }^{27}$ Taber (2000) allows for the possibility that the support of the error term could be bounded which allows for weaker support condition on the observables. 
(b) The distribution of $g_{c}\left(X_{c i}, x_{0}\right)$ conditional on $\left(X_{1 i}, X_{d i}, X_{0 i}\right)=\left(x_{1}, x_{d}, x_{0}\right)$ is stochastically dominated by the unconditional distribution of $g_{c}\left(X_{c i}, x_{0}\right)$.

This is a stochastic analogue of a support condition. In the case in which $X_{c i}$ were known at time one so that $X_{1 i}=X_{c i}$, this would be implied be a standard support condition. However, is general enough to allow for the distribution of $X_{c i}$ to not be known at time one, but we still need a time one variable $X_{1 i}$ that is useful in forecasting $X_{c i}$. For example $X_{c i}$ could be a variable like family income while the child is in college while $X_{1 i}$ is a variable like family income while the child is in high school. This assumption states that we can condition on the value of this variable so that the conditional probability that the agent chooses option $c$ in the second period can become arbitrarily small. In the family income example this means we could condition on families whose income while the child is in high school are sufficiently low that college seems like a very unlikely outcome for the child.

Assumption 7.3. $\left(\varepsilon_{1 i}, \varepsilon_{d i}, \varepsilon_{c i}\right)$ is independent of $\left(X_{1 i}, X_{d i}, X_{c i}, X_{0 i}\right)$, for any $\epsilon_{1} \in$ $\operatorname{supp}\left(\varepsilon_{1 i}\right)$,

$$
E\left(\left|\varepsilon_{c i}\right| \mid \varepsilon_{1 i}=\epsilon_{1}\right)<\infty
$$

and for any $\left(x_{1}, x_{d}, x_{0}\right) \in \operatorname{supp}\left(X_{1 i}, X_{d i}, X_{0 i}\right)$,

$$
E\left(\left|g_{c}\left(X_{c i}, x_{0}\right)\right| \mid\left(X_{1 i}, X_{d i}, X_{0 i}\right)=\left(x_{1}, x_{d}, x_{0}\right)\right)<\infty
$$

Assumption 7.3 is the separable independent assumption that we have been making throughout this chapter. We also need to assume that the stochastic components have finite expectations so that $V_{g}$ is finite.

Theorem 7.1. Under assumption 7.1,7.2, and 7.3, from data on $\left(X_{1 i}, X_{d i}, X_{c i}, X_{0 i}, J_{i}\right)$ $g_{d}$ and $g_{c}$ are identified up to monotonic transformation.

(Proof in Taber, 2000)

The basic strategy used in this proof is a stochastic extension of "identification at infinity." This should not be surprising as this looks very much like the type of selection problem we have discussed throughout this chapter: we can not observe the choice between $c$ and $h$ unless individuals have already rejected d. 
We identify $g_{c}$ in almost exactly the same was as we identified $g_{f}$ as presented for the Roy Model. With an exclusion restriction we can condition on $g_{d}$ arbitrarily low so that the probability of selecting node $d$ is close to zero. This leaves us with a simple binary choice model in which the agents choose between $h$ and $c$. The type of exclusion restriction used here is a variable that enters $g_{d}$, but does not influence $g_{c}$ directly. One can see this in the following expression

$$
\begin{aligned}
\lim _{g_{d}\left(x_{d}, x_{0}\right) \rightarrow-\infty} & \operatorname{Pr}\left(J_{i}=c \mid X_{i}=x\right) \\
& =\lim _{g_{d}\left(x_{d}, x_{0}\right) \rightarrow-\infty} \operatorname{Pr}\left[g_{d}\left(x_{d}, x_{0}\right)+\varepsilon_{d i} \leq V_{g}\left(x_{1}, x_{d}, x_{0}, \varepsilon_{1 i}\right), g_{c}\left(x_{c}, x_{0}\right)+\varepsilon_{c i}>0\right] \\
& =\operatorname{Pr}\left[g_{c}\left(x_{c}, x_{0}\right)+\varepsilon_{c i}>0\right] .
\end{aligned}
$$

Using standard identification strategies for the binary choice model described in the first step of identification of the Roy model, $g_{c}$ is identified.

Identification of $g_{d}$ is somewhat trickier but one can use essentially the same idea. In a static model one could use an identification at infinity argument by eliminating $c$ as an option and could compare the binary choice of $d$ versus $h$. In this stochastic case this is can not be done because the value of $X_{c i}$ is not known at time 1 . Thus we need a somewhat different type of exclusion restriction, a variable known at time one that does not enter $g_{d}$ directly, but does have predictive power for the distribution of $g_{c}$ above and beyond $X_{d i}$. To see how this works, suppose we have a variable $X_{1 i}$ that satisfies these conditions and that as $x_{1}$ gets small the conditional distribution of $g_{c}$ shifts to the left. In this case

$$
\lim _{x_{1} \rightarrow-\infty} E\left[\max \left(g_{c}\left(X_{c i}, x_{0}\right)+\varepsilon_{c i}, 0\right) \mid\left(X_{1 i}, X_{d i}, X_{0 i}\right)=\left(x_{1}, x_{d}, x_{0}\right), \varepsilon_{1 i}=\epsilon_{1}\right]=0,
$$

so that,

$$
\begin{aligned}
\lim _{x_{1} \rightarrow-\infty} & \operatorname{Pr}\left(J_{i}=d \mid X_{i}=x\right) \\
\quad= & \lim _{x_{1} \rightarrow-\infty} \operatorname{Pr}\left[g_{d}\left(x_{d}, x_{0}\right)+\varepsilon_{d i}>E\left[\max \left(V_{c i}, 0\right) \mid\left(X_{1 i}, X_{d i}, X_{0 i}\right)=\left(x_{1}, x_{d}, x_{0}\right), \varepsilon_{1 i}=\epsilon_{1}\right]\right] \\
\quad= & \operatorname{Pr}\left[g_{d}\left(x_{d}, x_{0}\right)+\varepsilon_{d i}>0\right] .
\end{aligned}
$$

From this piece we can identify $g_{d}$ up to a monotonic transformation. This type of variable will satisfy assumption 7.2. Note that the type of exclusion restriction we 
need here is something that is known at time 1 , is useful in forecasting $X_{c i}$, but does not affect $V_{d i}$.

Taber (2000) goes on to consider identification of the distribution of the error terms. The most general version of the full model above can not be identified without further assumptions so he instead studies a few interesting cases. Identification of the error terms requires a different kind of exclusion restriction. His key assumption requires variation in $g_{c}\left(x_{c}\right)$ holding $x_{1}$ fixed. Thus we need some uncertainty from the point of view of the agents. The full model is not identified if agent's have perfect information about future values of $X_{c i}$. A natural way to satisfy this exclusion restriction is with time varying observables. The details can be found in Taber (2000).

\subsection{Identification of the Components of the Bellman Equation}

While the model above is dynamic, we have not used Bellman's equation. A natural way to parameterize the model would be to define period specific utility functions $u_{h}\left(X_{h i}, X_{0 i}, \varepsilon_{h i}\right), u_{c}\left(X_{c i}, X_{0 i}, \varepsilon_{c i}\right)$, and $u_{g}\left(X_{1 i}, X_{0 i}, \varepsilon_{1 i}\right)$ in each of the three nodes above other than the dropout node. If we think of the model as a two period model we can define $u_{d}\left(t, X_{d i}, X_{0 i}, \varepsilon_{d i}\right)$ to be the period specific utility of individual $i$ if she drops out at time $t$. Conditional on graduating, she enters college if

$$
u_{c}\left(X_{c i}, X_{0 i}, \varepsilon_{c i}\right)>u_{h}\left(X_{h i}, X_{0 i}, \varepsilon_{h i}\right) .
$$

The Bellman equation for the high school graduate is

$$
\begin{aligned}
& V_{g}\left(x_{1}, x_{d}, x_{0}, \epsilon_{1}\right) \equiv u_{g}\left(x_{1}, x_{0}, \epsilon_{1}\right) \\
& \quad+\beta E\left[\max \left\{u_{c}\left(X_{c i}, X_{0 i}, \varepsilon_{c i}\right), u_{h}\left(X_{h i}, X_{0 i}, \varepsilon_{h i}\right)\right\} \mid\left(X_{1 i}, X_{d i}, X_{0 i}\right)=\left(x_{1}, x_{d}, x_{0}\right), \varepsilon_{1 i}=\epsilon_{1}\right]
\end{aligned}
$$

Mapping back to the notation in the subsection above, the rest of the value functions are defined as

$$
\begin{aligned}
& V_{d i}=u_{d}\left(1, X_{d i}, X_{0 i}, \varepsilon_{d i}\right)+\beta u_{d}\left(2, X_{d i}, X_{0 i}, \varepsilon_{d i}\right) \\
& V_{h i}=u_{g}\left(X_{1 i}, X_{0 i}, \varepsilon_{1 i}\right)+\beta u_{h}\left(X_{h i}, X_{0 i}, \varepsilon_{h i}\right) \\
& V_{c i}=u_{g}\left(X_{1 i}, X_{0 i}, \varepsilon_{1 i}\right)+\beta u_{c}\left(X_{c i}, X_{0 i}, \varepsilon_{c i}\right) .
\end{aligned}
$$

An obvious question arises as to whether one can separately identify the components of the value functions $\beta, u_{h}, u_{c}$, and $u_{d}$. Unfortunately, in general one can not do this. 
Consider a full certainty version of the model. In this case the decision of which occupation to enter would depend on $V_{d i}, V_{h i}$, and $V_{c i}$ only. One can choose any $\beta>0$ and any $u_{g}$, but then always find a value of $u_{c}$ and $u_{h}$ to leave $V_{c i}$ and $V_{h i}$ unchanged. For a simple model such as the one Taber (2000) presents, parameterizing the model in terms of the terminal value functions (i.e. $V_{d i}, V_{h i}$, and $V_{c i}$ ) avoids this problem as one does not need to decompose them into their components.

However, Taber's parameterization is clearly not feasible for an infinitely lived model. Furthermore, it is not convenient in an finite time model with many periods and state variables. It does not take advantage of the dimension reducing advantages of the Bellman formulation: the functions would depend on the whole history of state variables rather than just the current set.

Next we consider Rust's (1994) model. Note that we use his notation exactly even though it is inconsistent with our previous notation. Let $S_{i}$ represents the current state and $D_{i}$ represents the discrete choice. In general $S_{i}$ will contain elements that are both observed and unobserved by the econometrician. He writes the Bellman equation as

$$
v(s, d)=u(s, d)+\beta \int \max _{D_{i}^{\prime} \in D\left(S_{i}^{\prime}\right)}\left[v\left(S_{i}^{\prime}, D_{i}^{\prime}\right)\right] p\left(d S_{i}^{\prime} \mid S_{i}=s, D_{i}=d\right)
$$

where $v$ is the value function, $u$ is the period specific utility function, $\beta$ is the discount rate, $D(s)$ is the choice set in state of the world $s$, and $p$ is the transitional probability distribution of the state variables. Rust (1994) shows that one can not separately identify the model above from an alternative with the same $\beta$ and $p$, but with

$$
\bar{u}(s, d)=u(s, d)+f(s)-\beta \int f\left(S_{i}^{\prime}\right) p\left(d s^{\prime} \mid S_{i}=s, D_{i}=d\right) .
$$

Intuitively this is close to the discussion above in the simple model in which you can change the timing at which the innovation to utility takes place, without changing the value function.

Magnac and Thesmar (2002) discuss this issue in much greater detail. They not only show that the model is not identified, but document the extent of underidentification. They additionally assume that one can write

$$
u\left(S_{i}, d\right)=u_{d}\left(X_{i}\right)+\varepsilon_{d i}
$$

where $X_{i}$ is the observable part of the state space and the unobservable $\varepsilon_{d i}$ is mean independent of $x$ and independent across periods (conditional on $x$ and $d$ ). That is $S_{i}$ 
represents the state space, so if one knows $S_{i}$, they also know $X_{i}$ and $\varepsilon_{d i}$. They show that given knowledge of $\beta$ and the joint distribution of the $\varepsilon_{d i}$, one can identify

$$
\begin{aligned}
U_{d}(x) \equiv u_{d}(x)+ & \beta \int \max _{D_{i}^{\prime} \in D\left(D_{i}^{\prime}\right)}\left[v\left(S_{i}^{\prime}, D_{i}^{\prime}\right)\right] p\left(d S_{i}^{\prime} \mid X_{i}=x, D_{i}=d\right)-u_{k}(x) \\
& +\beta \int \max _{D_{i}^{\prime} \in D\left(S_{i}^{\prime}\right)}\left[v\left(S_{i}^{\prime}, D_{i}^{\prime}\right)\right] p\left(d S_{i}^{\prime} \mid X_{i}=x, D_{i}=k\right)
\end{aligned}
$$

where $k$ is one of the elements of $D(s)$. They further explore the model with additional identifying information and correlated random effects.

How problematic it is that the model is not fully identified? The answer to this question depends on the purpose of the model. That is, even if the model is not fully identified, one may still be able to identify policy counterfactuals of interest. Ichimura and Taber (2002) provide one example of a case in which the policy counterfactual can be identified. They start with the model of Keane and Wolpin (2001) and show how one can estimate a semiparametric reduced form version of this model and use it to evaluate the effect of a tuition subsidy on college enrollment. They key is having enough structure on the model to map variation in the data to the counterfactual tuition subsidy.

Aguirregabiria (2010) presents a different and somewhat more general example of policy evaluation in a finite time dynamic discrete choice model. We do not get into the details as it is different from the types of labor models we study here, but he shows that despite the fact that his full model is not identified, the welfare effect function resulting from the policy change can be identified. Thus one can do welfare analysis even though the full model is not identified.

\subsection{Dynamic Generalized Roy Model}

Heckman and Navarro (2007) provide another example showing that one can identify interesting counterfactuals even when the full model is not identified. Their study complements the discussion in this chapter as it extends the work on identification in dynamic discrete choice models into the treatment effects literature discussed in section 5 above. They consider a finite time optimal stopping problem. Using the notation used above in section $7.2, D_{i}$ is either zero or one, and once it is one it remains 1 forever. Their main example is a schooling model in which students decide at which time to leave school (assuming that after leaving they cannot come back). The model 
is essentially a dynamic generalized Roy model. Let $T_{i a}$ and $L_{i a}$ respectively denote the level of schooling and a dummy for whether individual $i$ is out of school at age $a$. Using a somewhat modified version of their notation we can write time $a$ earnings as

$$
Y_{i, a, t, \ell}=\mu\left(a, t, \ell, X_{i}\right)+\varepsilon_{i, a, t, \ell}
$$

where $t$ and $\ell$ represent potential outcomes of $T_{i, a}$ and $L_{i, a}$. Heckman and Navarro (2007) also assume that the cost of schooling can be written as

$$
C_{i, t}=\Phi\left(t, X_{i}, Z_{i}\right)+\omega_{i, t}
$$

In order to keep our notation complete and consistent across sections we will assume that random variable $\Theta_{i, a}$ summarizes all information (both observables and unobservables) that individual $i$ has at age $a$. This means that if we know $\Theta_{i, a}$ we also know $\left(X_{i}, Z_{i}, T_{i, a}, L_{i, a}, \varepsilon_{i, a, t, l}, \omega_{i, t}\right)$, so when we condition on $\Theta_{i, a}=\theta$, we are conditioning on $\left(X_{i}, Z_{i}, T_{i, a}, L_{i, a}, \varepsilon_{i, a, t, l}, \omega_{i, t}\right)=\left(x, z, t, \ell, \epsilon_{a, t, \ell}, \omega_{t}\right)$. We will make use of this notation below.

Once a student leaves school they make no further decisions, so if a student leaves school at age $a$ with $t$ years of schooling, lifetime utility discounted to the time one leaves school is written as

$$
R(a, t, \theta)=E\left(\sum_{j=0}^{\bar{T}}\left(\frac{1}{1+r}\right)^{j} Y_{i, a+j, t, 1} \mid \Theta_{i, a}=\theta\right) .
$$

The only decision that agents make is whether they will drop out of school or not. For a student at age $a$ with $t$ years of schooling the value function when they make this decision is written as

$$
\begin{aligned}
& V(a, t, \theta)=\max \{R(a, t, \theta), \\
& \quad \mu(a, t, 0, x)+\epsilon_{a, t, 0}-\Phi(t, x, z)-\omega_{t}+\left(\frac{1}{1+r}\right) E\left[V\left(a+1, t+1, \Theta_{i, a+1} \mid \Theta_{i, a}=\theta\right]\right\} .
\end{aligned}
$$

This is basically a dynamic version of the generalized Roy model. Identification follows by essentially combining the arguments used by Taber (2000) for the dynamic aspects of the model with the arguments for identification of the generalized Roy model. Heckman and Navarro (2007) use higher level assumptions to avoid the use of exclusion 
restrictions. $^{28}$ They also use a factor structure on the distribution of the error term to reduce dimension. We refer readers interested in these generalizations and in the details of their proof to their paper. Here we attempt to give an intuitive feel for identification of this model and show how it is related to identification of the generalized Roy model presented in section 3.3.

\section{Identification of reduced form choice model}

In this case they do not derive an explicit reduced form, but note that

$$
\operatorname{Pr}\left(T_{i, a}=t \mid X_{i}=x, Z_{i}=z\right)
$$

can be identified directly from the data.

\section{Identification of the Earnings Equation $\mu$}

With exclusion restrictions this can be done in exactly the same way as in the static model. Assuming that $\varepsilon_{i, a, t, \ell}$ has a zero mean,

$$
\begin{array}{r}
\lim _{\operatorname{Pr}\left(T_{i, a}=t \mid\left(X_{i}, Z_{i}\right)=(x, z)\right) \rightarrow 1} E\left[Y_{i, a+j, t, 1} \mid\left(X_{i}, Z_{i}\right)=(x, z)\right]=\mu(a+j, t, 1, x) . \\
\lim _{\operatorname{Pr}\left(T_{i, a}>t \mid\left(X_{i}, Z_{i}\right)=(x, z)\right) \rightarrow 1} E\left[Y_{i, a, a, 0} \mid\left(X_{i}, Z_{i}\right)=(x, z)\right]=\mu(a, a, 0, x) .
\end{array}
$$

Thus this is a version of an "identification at infinity argument." Heckman and Navarro (2007) do not use this explicit argument because they avoid exclusion restrictions with a higher order assumption. However, they do use identification at infinite.

\section{Identification of $\Phi$}

Next consider the identification of the cost of schooling function $\Phi$. The best way to think about identification in these types of models is to start with the final period and work backward.

Since the maximum length of schooling is $\bar{T}$, the final decision is made when the individual has $\bar{T}-1$ years of schooling. At that point the student decides whether to attend the final year of school or not. Heckman and Navarro (2007) use an "identification at infinity" argument so that $\operatorname{Pr}\left(T_{i}>\bar{T}-2 \mid X_{i}=x, Z_{i}=z\right) \approx 1$. Then the

\footnotetext{
${ }^{28}$ This relates back to our discussion of identification and exclusion restrictions in the sample selection model at the very end of section 3. Exclusion restrictions prevent one from setting $\widetilde{g}_{f}(x)=g_{f}(x)+h(g(x))$ but shape restrictions on $g$ and $g_{f}$ can do this as well. Their "higher level assumptions" are essentially assuming that we make restrictions on $g_{f}$ so that we can not add $h(g(x))$ to it and remain in the permissible class of $g_{f}$ functions.
} 
problem becomes analogous to a static problem. ${ }^{29}$ That is

$$
\begin{aligned}
\lim _{\operatorname{Pr}\left(T_{i}>\bar{T}-2 \mid X_{i}=x, Z_{i}=z\right) \rightarrow 1} & \operatorname{Pr}\left(T_{i \bar{T}}=\bar{T} \mid X_{i}=x, Z_{i}=z\right) \\
=\operatorname{Pr}( & R\left(\bar{T}-1, \bar{T}-1, \Theta_{i, \bar{T}-1}\right)<\mu(\bar{T}-1, \bar{T}-1,0, x)+\varepsilon_{i, \bar{T}-1, \bar{T}-1,0} \\
& \left.\quad \Phi(\bar{T}-1, x, z)-\omega_{i, \bar{T}-1}+\left(\frac{1}{1+r}\right) E\left[R\left(\bar{T}, \bar{T}, \Theta_{i \bar{T}}\right) \mid \Theta_{i, \bar{T}-1}\right] \mid X_{i}=x, Z_{i}=z\right) .
\end{aligned}
$$

This is analogous to identification of the $g_{h}$ function in the Roy model. ${ }^{30}$

Now one can just iterate backward given knowledge of all variables at $\bar{T}$ and $\bar{T}-1$. That is, the distribution of $\left(\frac{1}{1+r}\right) E\left[V\left(\bar{T}-1, \bar{T}-1, \Theta_{i, \bar{T}-1} \mid \Theta_{i, \bar{T}-2}\right]\right.$ has been identified so once again we can use the identification approach of the static problem and can use the same basic style of proof. That is we can condition on a set of variables so that $\operatorname{Pr}\left(t>\bar{T}-2 \mid X_{i}=x, Z_{i}=z\right) \approx 1$ so that identification is analogous to the static problem. Consider the decision with $\bar{T}-2$ years of schooling.

$$
\begin{aligned}
\lim _{\operatorname{Pr}\left(T_{i}>\bar{T}-3 \mid X_{i}=x, Z_{i}=z\right) \rightarrow 1} & \operatorname{Pr}\left(T_{i, \bar{T}-1}=\bar{T}-1 \mid X_{i}=x, Z_{i}=z\right) \\
=\operatorname{Pr}( & R\left(\bar{T}-2, \bar{T}-2, \Theta_{i, \bar{T}-2}\right)<\mu(\bar{T}-2, \bar{T}-2,0, x)+\varepsilon_{i, \bar{T}-2, \bar{T}-2,0}-\Phi(\bar{T}-2, x, z) \\
& \quad-\omega_{i, \bar{T}-2}+\left(\frac{1}{1+r}\right) E\left[V\left(\bar{T}-1, \bar{T}-1, \Theta_{i, \bar{T}-1} \mid \Theta_{i, \bar{T}-2}\right] \mid X_{i}=x, Z_{i}=z\right) .
\end{aligned}
$$

One can keep iterating on this procedure so that $\Phi$ is identified in all periods.

Identification of the Distribution of the Error Terms

Heckman and Navarro (2007) impose a factor structure so that

$$
\begin{aligned}
\varepsilon_{i, a, t, \ell} & =\alpha_{a, t, \ell}^{\prime} \tau_{i}+\varepsilon_{i, a, t, \ell} \\
\omega_{i, t} & =\lambda_{t}^{\prime} \tau_{i}+\xi_{i, t}
\end{aligned}
$$

where $\tau_{i}$ is a vector random variable, the $\varepsilon^{\prime} s$ and $\xi^{\prime} s$ are all independently distributed, and the $\alpha$ and $\lambda$ terms are factor loadings. Given this structure and that the other components of the model have been identified, identification of the distribution of the

\footnotetext{
${ }^{29}$ Once again, Heckman and Navarro (2007) use higher order assumptions that do not require exclusion restrictions. For example they allow for either an exclusion restriction or a cost variable to identify the scale (such as tuition described in section 4 above).

${ }^{30}$ Note that we have violated one convention in this chapter which is to make conditioning explicit such as $E\left(\cdot \mid X_{i}=x\right)$. When we condition on $\Theta_{i, \bar{T}-1}$ we cannot do this explicitly because while the expectation inside the expression conditions on its outcome, the probability expression (immediately after the $=$ sign) treats $\Theta_{i, \bar{T}-1}$ as a random variable.
} 
error terms and factor loadings can be done by varying the indices in much the same way as in the static model. We do not show this explicitly.

\section{Conclusions}

In this chapter we have presented identification results for models of the labor market. The main issue in all of these models is the issue of sample selection bias. We start with the classic Roy model and devote much space to explaining how this model can be identified. We then show how these results can be extended to more complicated cases, the generalized Roy model, treatment effect models, duration data, search models, and forward looking dynamic models. We show the importance of both exclusion restrictions and support conditions for all of these models.

\section{Technical Appendix}

\section{Proof of Theorem 2.1}

Let $\mathcal{X}^{*}$ be the set of points $\left(x^{c}, x^{d}\right)$ at which $g$ is continuous in $x^{c}$. For any $\left(x^{c}, x^{d}\right) \in \mathcal{X}^{*}$ and $\delta>0, E\left(Y_{i} \mid\left\|X_{i}^{c}-x^{c}\right\|<\delta, X_{i}^{d}=x^{d}\right)$ is identified directly from the data.

Since $g$ is continuous at $\left(x^{c}, x^{d}\right)$,

$$
\lim _{\delta \downarrow 0} E\left(Y_{i} \mid\left\|X_{i}^{c}-x^{c}\right\|<\delta, X_{i}^{d}=x^{d}\right)=g\left(x^{c}, x^{d}\right),
$$

so $g\left(x^{c}, x^{d}\right)$ is identified on $\mathcal{X}^{*}$. By assumption $2.2, \mathcal{X}^{*}$ has measure one.

\section{Proof of Theorem 3.1}

Let $\mathcal{X}^{*}$ be the set of points $\left(x_{f}^{c}, x_{f}^{d}, x_{h}^{c}, x_{h}^{d}, x_{0}^{c}, x_{0}^{d}\right)$ at which $g_{h}$ and $g_{f}$ are continuous in $x^{c}$.

First notice that for any $x=\left(x_{f}^{c}, x_{f}^{d}, x_{h}^{c}, x_{h}^{d}, x_{0}^{c}, x_{0}^{d}\right) \in \mathcal{X}^{*}$,

$$
\begin{aligned}
\lim _{\delta \downarrow 0} \operatorname{Pr}\left(J_{i}=f \mid\left\|X_{i}^{c}-x^{c}\right\|<\delta, X_{i}^{d}=x^{d}\right) & \equiv \operatorname{Pr}\left(J_{i}=f \mid X_{i}=x\right) \\
& =g(x)
\end{aligned}
$$

is identified. 
Thus we have thus established that we can write the model as $J_{i}=f$ if and only if $g\left(X_{i}\right)>\varepsilon_{i}$ where $\varepsilon_{i}$ is uniform $[0,1]$ and that $g$ is identified.

Next consider identification of $g_{f}$ at the point $\left(x_{f}, x_{0}\right)$. This is basically the standard selection problem. As long as $g$ is continuous on the continuous covariates at this point, we can identify

$$
\begin{aligned}
& \lim _{\delta \downarrow 0} \operatorname{Med}\left(Y_{i}\left|\left\|X_{f i}^{c}-x_{f}^{c}\right\|<\delta, X_{f i}^{d}=x_{f}^{d},\left\|X_{0 i}^{c}-x_{0}^{c}\right\|<\delta, X_{0 i}^{d}=x_{0}^{d},\right| 1-g\left(X_{i}\right) \mid<\delta, J_{i}=f\right) . \\
& =g_{f}\left(x_{f}, x_{0}\right)+ \\
& \quad \lim _{\delta \downarrow 0} \operatorname{Med}\left(\varepsilon_{f i}\left|\left\|X_{f i}^{c}-x_{f}^{c}\right\|<\delta, X_{f i}^{d}=x_{f}^{d},\left\|X_{0 i}^{c}-x_{0}^{c}\right\|<\delta, X_{0 i}^{d}=x_{0}^{d},\right| 1-g\left(X_{i}\right) \mid<\delta, J_{i}=f\right) \\
& =g_{f}\left(x_{f}, x_{0}\right) .
\end{aligned}
$$

Thus $g_{f}$ is identified. Note that having an exclusion restriction with strong support conditions is necessary to guarantee that the measure of the set of $X_{i}$ satisfying $\left|1-g\left(X_{i}\right)\right|<\delta$ is not zero.

Next we show how to identify $g_{h}$. Note that for any $\left(x_{h}, x_{0}\right)$ where $g$ is continuous in the continuous covariates and $\delta>0$ we can identify the set

$$
\mathcal{X}\left(x_{h}, x_{0}, \delta\right) \equiv\left\{\widetilde{x} \in \mathcal{X}^{*}:\left\|\tilde{x}_{h}^{c}-x_{h}^{c}\right\|<\delta, \tilde{x}_{h}^{d}=x_{f}^{d},\left\|\tilde{x}_{0}^{c}-x_{0}^{c}\right\|<\delta, \tilde{x}_{0 i}^{d}=x_{0}^{d},|0.5-g(\widetilde{x})|<\delta\right\}
$$

where $\tilde{x}=\left(\tilde{x}_{f}, \tilde{x}_{h}, \tilde{x}_{0}\right)$. Under our assumptions it has positive measure.

The median zero assumption guarantees that

$$
\begin{aligned}
\lim _{\delta \downarrow 0} \mathcal{X}\left(x_{h}, x_{0}, \delta\right) & =\left\{\tilde{x} \in \mathcal{X}^{*}: \tilde{x}_{h}=x_{h}, \tilde{x}_{0}=x_{0}, 0.5=\operatorname{Pr}\left(J_{i}=F \mid X_{i}=\tilde{x}\right)\right\} \\
& =\left\{\tilde{x} \in \mathcal{X}^{*}: \tilde{x}_{h}=x_{h}, \tilde{x}_{0}=x_{0}, 0.5=\operatorname{Pr}\left(\varepsilon_{h i}-\varepsilon_{f i} \leq g_{f}\left(\tilde{x}_{f}, x_{0}\right)-g_{h}\left(x_{h}, x_{0}\right)\right)\right\} \\
& =\left\{\tilde{x} \in \mathcal{X}^{*}: \tilde{x}_{h}=x_{h}, \tilde{x}_{0}=x_{0}, g\left(\tilde{x}_{f}, x_{0}\right)=g_{h}\left(x_{h}, x_{0}\right)\right\}
\end{aligned}
$$

is identified. Since $g\left(\tilde{x}_{f}, x_{0}\right)$ is identified, $g_{h}$ is identified.

Finally consider identification of $G$ given $g_{f}$ and $g_{h}$. Note that from the data one can identify

$$
\begin{aligned}
& \lim _{\delta \downarrow 0} \operatorname{Pr}\left(J_{i}=f, \log \left(Y_{f i}\right)<s \mid\left\|X_{i}^{c}-x^{c}\right\|<\delta, X_{i}^{d}=x^{d}\right) \\
= & \lim _{\delta \downarrow 0} \operatorname{Pr}\left(g_{h}\left(X_{h i}, X_{0 i}\right)+\varepsilon_{h i} \leq g_{f}\left(X_{f i}, X_{0 i}\right)+\varepsilon_{f i}, g_{f}\left(X_{f i}, X_{0 i}\right)+\varepsilon_{f i} \leq s \mid\left\|X_{i}^{c}-x^{c}\right\|<\delta, X_{i}^{d}=x^{d}\right) \\
= & \operatorname{Pr}\left(\varepsilon_{h i}-\varepsilon_{f i} \leq g_{f}\left(x_{f}, x_{0}\right)-g_{h}\left(x_{h}, x_{0}\right), \varepsilon_{f i} \leq s-g_{f}\left(x_{f}, x_{0}\right)\right)
\end{aligned}
$$


which is the cumulative distribution function of $\left(\varepsilon_{h i}-\varepsilon_{f i}, \varepsilon_{f i}\right)$ evaluated at the point $\left(g_{f}\left(x_{f}, x_{0}\right)-g_{h}\left(x_{h}, x_{0}\right), s-g_{f}\left(x_{f}, x_{0}\right)\right)$. By varying the point of evaluation one can identify the joint distribution of $\left(\varepsilon_{h i}-\varepsilon_{f i}, \varepsilon_{f i}\right)$ from which one can derive the joint distribution of $\left(\varepsilon_{f i}, \varepsilon_{h i}\right)$.

\section{Proof of Theorem 4.1}

As in the proof of Theorem 3.1, let $\mathcal{X}^{*}$ be the set of points $\left(z^{c}, z^{d}, x_{f}^{c}, x_{f}^{d}, x_{h}^{c}, x_{h}^{d}, x_{0}^{c}, x_{0}^{d}\right)$ at which $g_{h}, g_{f}, \varphi_{h}$ and $\varphi_{f}$ are continuous in $\left(z^{c}, z^{d}, x_{f}^{c}, x_{f}^{d}, x_{h}^{c}, x_{h}^{d}, x_{0}^{c}, x_{0}^{d}\right)$.

First notice that for any $(z, x)=\left(z^{c}, z^{d}, x_{f}^{c}, x_{f}^{d}, x_{h}^{c}, x_{h}^{d}, x_{0}^{c}, x_{0}^{d}\right) \in \mathcal{X}^{*}$,

$$
\begin{aligned}
& \lim _{\delta \downarrow 0} \operatorname{Pr}\left(J_{i}=f \mid\left\|X_{i}^{c}-x^{c}\right\|<\delta,\left\|Z_{i}^{c}-z^{c}\right\|<\delta,\left(Z_{i}^{d}, X_{i}^{d}\right)=\left(z^{d}, x^{d}\right)\right) \\
& \quad=\operatorname{Pr}\left(\nu_{i} \leq \varphi(z, x)\right) \\
& \quad=\varphi(z, x) .
\end{aligned}
$$

Thus $\varphi$ is identified on the relevant set. Next consider $g_{f}$ and the joint distribution of $\left(\nu_{i}, \varepsilon_{f i}\right)$. Note that for all $\left(z, x_{f}, x_{h}, x_{0}\right) \in \mathcal{X}^{*}$ and any $y \in \mathbb{R}$, we can identify

$$
\begin{aligned}
& \lim _{\delta \downarrow 0} \operatorname{Pr}\left(J_{i}=f, Y_{f i} \leq y \mid\left\|X_{i}^{c}-x^{c}\right\|<\delta,\left\|Z_{i}^{c}-z^{c}\right\|<\delta,\left(Z_{i}^{d}, X_{i}^{d}\right)=\left(z^{d}, x^{d}\right)\right) \\
& \quad=\operatorname{Pr}\left(\nu_{i} \leq \varphi(z, x), g_{f}\left(x_{f}, x_{0}\right)+\varepsilon_{f i} \leq y\right)
\end{aligned}
$$

which is the joint distribution of $\left(\nu_{i}, g_{f}\left(x_{f}, x_{0}\right)+\varepsilon_{f i}\right)$ evaluated at $(\varphi(z, x), y)$. Holding $\left(x_{f}, x_{0}\right)$ constant and varying $(\varphi(z, x), y)$ we can estimate this joint distribution. Since the median of $\varepsilon_{f i}$ is zero, $g_{f}$ is identified and given $g_{f}$ the joint distribution of $\left(\nu_{i}, \varepsilon_{f i}\right)$ is identified. Since the model is symmetric in $h$ and $f, g_{h}$ and the joint distribution of $\left(\nu_{i}, \varepsilon_{h i}\right)$ are identified using the analogous argument.

\section{Proof of Theorem 4.2}

The first part is analogous to step three of identification of the Roy model presented in the text. Note that for any $\left(z, x_{0}\right)$ and $\delta$ we can identify the set

$$
\left.\mathcal{X}\left(z, x_{0}, \delta\right) \equiv\left\{(\tilde{z}, \tilde{x}) \in \mathcal{X}^{*}:\left\|\tilde{z}^{c}-z^{c}\right\|<\delta, \tilde{z}^{d}=z^{d},\left\|\tilde{x}_{0}^{c}-x_{0}^{c}\right\|<\delta, \tilde{x}_{0}^{d}=x_{0}^{d}, \mid 0.5-\varphi(\tilde{z}, \tilde{x})\right) \mid<\delta\right\}
$$


and it has positive measure where the elements of $(\tilde{z}, \tilde{x})$ are defined in the obvious way. The median zero assumption guarantees that

$$
\begin{aligned}
& \lim _{\delta \downarrow 0} \mathcal{X}\left(z, x_{0}, \delta\right)=\left\{(\tilde{z}, \tilde{x}) \in \mathcal{X}^{*}: \tilde{z}=z, \tilde{x}_{0}=x_{0}, 0.5=\operatorname{Pr}\left(J_{i}=F \mid\left(Z_{i}, X_{i}\right)=(\tilde{z}, \tilde{x})\right)\right\} \\
& \quad=\left\{(\tilde{z}, \tilde{x}) \in \mathcal{X}^{*}: \tilde{z}=z, \tilde{x}_{0}=x_{0}, 0.5=\operatorname{Pr}\left(\varepsilon_{h i}-\varepsilon_{f i} \leq g_{f}\left(\tilde{x}_{f}, x_{0}\right)+\varphi\left(z, x_{0}\right)-g_{h}\left(\tilde{x}_{h}, x_{0}\right)\right)-\varphi\left(z, x_{0}\right)\right\} \\
& \quad=\left\{(\tilde{z}, \tilde{x}) \in \mathcal{X}^{*}: \tilde{z}=z, \tilde{x}_{0}=x_{0}, \varphi_{f}\left(z, x_{0}\right)-\varphi_{h}\left(z, x_{0}\right)=g_{h}\left(\tilde{x}_{h}, x_{0}\right)-g_{f}\left(\tilde{x}_{f}, x_{0}\right)\right\}
\end{aligned}
$$

Since $g_{h}$ and $g_{f}$ are identified by Theorem 4.1, $\varphi_{f}\left(z, x_{0}\right)-\varphi_{h}\left(z, x_{0}\right)$ is also identified. Given this we can identify the distribution of $\left(\varepsilon_{h i}+\nu_{h i}-\varepsilon_{f i}-\nu_{f i}, \varepsilon_{f i}\right)$ and $\left(\varepsilon_{h i}+\nu_{h i}-\varepsilon_{f i}-\nu_{f i}, \varepsilon_{h i}\right)$ since in general

$$
\begin{aligned}
& \lim _{\delta \downarrow 0} \operatorname{Pr}\left(J_{i}=f, Y_{f i} \leq y \mid\left\|Z_{i}^{c}-z^{c}\right\|<\delta, Z_{i}^{d}=z^{d},\left\|X_{i}^{c}-x^{c}\right\|<\delta, X_{i}^{d}=x_{0}^{d}\right) \\
& \quad=\operatorname{Pr}\left(\varepsilon_{h i}+\nu_{h i}-\varepsilon_{f i}-\nu_{f i} \leq g_{f}\left(x_{f}, x_{0}\right)+\varphi_{f}\left(z, x_{0}\right)-g_{h}\left(x_{h}, x_{0}\right)-\varphi_{h}\left(z, x_{0}\right), \varepsilon_{f i} \leq y-g_{f}\left(x_{f}, x_{0}\right)\right)
\end{aligned}
$$

and

$$
\begin{aligned}
& \lim _{\delta \downarrow 0} \operatorname{Pr}\left(J_{i}=r, Y_{h i} \leq y \mid\left\|Z_{i}^{c}-z^{c}\right\|<\delta, Z_{i}^{d}=z^{d},\left\|X_{i}^{c}-x^{c}\right\|<\delta, X_{i}^{d}=x_{0}^{d}\right) \\
& \quad=\operatorname{Pr}\left(-\left(\varepsilon_{h i}+\nu_{h i}-\varepsilon_{f i}-\nu_{f i}\right) \leq g_{h}\left(x_{h}, x_{0}\right)+\varphi_{h}\left(z, x_{0}\right)-g_{f}\left(x_{f}, x_{0}\right)-\varphi_{f}\left(z, x_{0}\right), \varepsilon_{h i} \leq y-g_{h}\left(x_{h}, x_{0}\right)\right)
\end{aligned}
$$

\section{Proof of Theorem 5.1}

Theorem 4.1 shows that the marginal distributions of $\varepsilon_{f i}$ and $\varepsilon_{h i}$ are identified. Since their expectations are finite, $E\left(\varepsilon_{f i}\right)$ and $E\left(\varepsilon_{h i}\right)$ are identified. We also showed that $g_{f}$ and $g_{h}$ are identified over a set of measure 1. Note that $E\left(\pi_{i}\right)=E\left(Y_{f i}\right)-E\left(Y_{h i}\right)=$ $E\left(g_{f}\left(X_{f i}, X_{0 i}\right)+\varepsilon_{f i}\right)-E\left(g_{h}\left(X_{h i}, X_{0 i}\right)+\varepsilon_{h i}\right)=g_{f}\left(X_{f i}, X_{0 i}\right)-g_{h}\left(X_{h i}, X_{0 i}\right)+E\left(\varepsilon_{f i}\right)-$ $E\left(\varepsilon_{h i}\right)$. Because all the components of $E\left(\pi_{i}\right)$ are identified, $E\left(\pi_{i}\right)$ is identified as well.

\section{Proof of Theorem 5.2}

The marginal distribution of $X_{i}$, the joint distribution of $\left(X_{i}, Y_{f i}\right)$ conditional on $J_{i}=f$ and the joint distribution of $\left(X_{i}, Y_{h i}\right)$ conditional on $J_{i}=h$ are identified directly from the data. Assumption 5.2 guarantees that for both fishing and hunting $(j \in\{f, h\})$, 
the conditional distribution of $Y_{j i}$ conditional on $X_{i}$ and $J_{i}=j$ is the same as the conditional distribution of $Y_{j i}$ conditional on $X_{i}$ alone. From each of these conditional distributions and the marginal distribution of $X_{i}$, one can identify $E\left(Y_{j i}\right)$ and thus the average treatment effect is identified by taking the difference between the two.

\section{Proof of Theorem 6.2}

Let $\mathcal{X}^{*}$ be the set of points $\left(x^{c}, x^{d}\right)$ at which the functions are all continuous in $x^{c}$.

First note that in this model the hazard rate of finding for any individual can be written as

$$
\phi\left(X_{\lambda i}, X_{0 i}\right) \nu_{i}\left[1-F_{i \varepsilon_{f}}\left(\log \left(W_{i}^{r}\right)-g_{f}\left(X_{f i}, X_{0 i}\right)\right)\right] .
$$

Our first goal is for any $\left(x_{f}, x_{\lambda}, x_{0}\right) \in \mathcal{X}^{*}$, to identify the values of $x_{h}$ that send $g_{h}\left(x_{h}, x_{0}\right)$ arbitrarily large so that all offers are accepted. Since the reservation wage is strictly decreasing in $g_{h}$, the hazard rate is strictly increasing in $g_{h}$, we can do this by fixing $\left(X_{f i}, X_{0 i}\right)$ within some neighborhood of $\left(x_{f}, x_{0}\right)$ and finding the value of $x_{h}$ that minimizes the job finding rate.

More formally for any $\left(x_{f}, x_{\lambda}, x_{0}\right)$ and $\delta$, define

$$
x_{h}(\delta) \equiv \operatorname{argmin} E\left(T_{i} \mid\left\|X_{i}^{c}-\left(x_{f}^{c}, x_{h}^{c}(\delta), x_{\lambda}^{c}, x_{0}^{c}\right)\right\|<\delta,\left(X_{i}^{d}=\left(x_{f}^{d}, x_{h}^{d}(\delta), x_{\lambda}^{d}, x_{0}^{d}\right)\right)\right) .
$$

Note that this minimum will be such that as $\delta \rightarrow 0, W_{i}^{r} \rightarrow 0$ so that

$$
\begin{gathered}
\lim _{\delta \downarrow 0} \operatorname{Pr}\left(\log \left(T_{i}\right)<t, \log \left(W_{f i t}\right)<w\|\| X_{i}^{c}-\left(x_{f}^{c}, x_{h}^{c}(\delta), x_{\lambda}^{c}, x_{0}^{c}\right) \|<\delta, X_{i}^{d}=\left(x_{f}^{d}, x_{h}^{d}(\delta), x_{\lambda}^{d}, x_{0}^{d}\right)\right) \\
=G_{\omega^{*}, \varepsilon}\left(t+\log \left(\phi\left(x_{\lambda}, x_{0}\right)\right), w-g_{f}\left(x_{f}, x_{0}\right)\right)
\end{gathered}
$$

where $G$ is the joint distribution between a convolution of $\omega_{i t}$ and an extreme value and of $\varepsilon_{\text {fit }}$. Given $G$, applying the identification arguments for the mixed proportional hazard model one can identify $\phi$. Furthermore, $g_{f}$ can be identified through the standard argument for identification of the regression model.

Finally, recovering $g_{h}$ can be done in an analogous was as for the Roy model. Notice that the reservation wage is scalable so that if we increase both $C_{i}$ and $W_{i t}$ by $10 \%$, then the reservation wage increases by $10 \%$ and the probability of job acceptance does not change. That is for any $\delta>0$ if $w_{i}^{*}$ solves

$$
e^{g_{h}\left(X_{h i}, X_{0 i}\right)+\varepsilon_{h i}}+w_{i}^{*}=\frac{\lambda_{i}}{r} \int_{\log \left(w_{i}^{*}\right)-g_{f}\left(X_{f i}, X_{0 i}\right)}^{\infty}\left(e^{g_{f}\left(X_{f i}, X_{0 i}\right)+\varepsilon_{f i t}}-w_{i}^{*}\right) d F_{i \varepsilon_{f}}\left(\varepsilon_{f i t}\right)
$$


then $w_{i}^{*} e^{\delta}$ solves

$$
e^{g_{h}\left(X_{h i}, X_{0 i}\right)+\delta+\varepsilon_{h i}}+w_{i}^{*} e^{\delta}=\frac{\lambda_{i}}{r} \int_{\log \left(w_{i}^{*}\right)-g_{f}\left(X_{f i}, X_{0 i}\right)}^{\infty}\left(e^{g_{f}\left(X_{f i}, X_{0 i}\right)+\delta+\varepsilon_{f i t}}-w_{i}^{*} e^{\delta}\right) d F_{i \varepsilon_{f}}\left(\varepsilon_{f i t}\right),
$$

but the probability of accepting a job and thus the expected duration remains the same.

Thus as in the identification of the slope that we discuss in Step 2 of identification of the Roy model, for any $\left(x_{h}, x_{0}\right)$ and $\left(\tilde{x}_{h}, \tilde{x}_{0}\right)$ suppose we want to identify $g_{h}\left(x_{h}, x_{0}\right)-$ $g_{h}\left(\tilde{x}_{h}, \tilde{x}_{0}\right)$. Fix $x_{\lambda}$ and $\tilde{x}_{\lambda}$ so that $\phi\left(x_{\lambda}, x_{0}\right)=\phi\left(\tilde{x}_{\lambda}, \tilde{x}_{0}\right)$. Then the key here is finding values $x_{f}$ and $\tilde{x}_{f}$ so that

$\lim _{\delta \downarrow 0} E\left(\log \left(Z\left(T_{i}\right)\right) \mid\left\|X_{i}^{c}-x^{c}\right\|<\delta, X_{i}^{d}=x^{d}\right)=\lim _{\delta \downarrow 0} E\left(\log \left(Z\left(T_{i}\right)\right) \mid\left\|X_{i}^{c}-\tilde{x}^{c}\right\|<\delta, X_{i}^{d}=\tilde{x}^{d}\right)$

But if this is the case it must be that

$$
g_{f}\left(x_{f}, x_{0}\right)-g_{h}\left(x_{h}, x_{0}\right)=g_{f}\left(\tilde{x}_{f}, \tilde{x}_{0}\right)-g_{h}\left(\tilde{x}_{h}, \tilde{x}_{0}\right)
$$

but then

$$
g_{h}\left(x_{h}, x_{0}\right)-g_{h}\left(\tilde{x}_{h}, \tilde{x}_{0}\right)=g_{f}\left(x_{f}, x_{0}\right)-g_{f}\left(\tilde{x}_{f}, \tilde{x}_{0}\right)
$$

where the right hand side has already been identified. Thus $g_{h}$ is identified up to location on the set $\mathcal{X}^{*}$. 


\section{References}

Abbring, J., 2010, "Identification of Dynamic Discrete Choice Models," forthcoming Annual Review of Economics.

Abbring, J., and G. Ridder, 2009, "A Note on the Non-parametric Identification of Generalized Accelerated Failure-Time Models," unpublished manuscript, Tilburg University.

Abbring, J., and J. Heckman, 2007, "Econometric Evaluation of Social Programs, Part III: Distributional Treatment Effects, Dynamic Treatment Effects, Dynamic Discrete Choice, and General Equilibrium Policy Evaluation," Handbook of Econometrics, Heckman and Leamer eds., North Holland, Amsterdam, 5145-5303.

Abbring, J., and G. van den Berg, 2003, "The Identifiability of the Mixed Proportional Hazards Competing Risks Model," Journal of the Royal Statical Society, Series B (Statistical Methodology)," 3, 701-710.

Aguirregabiria, V., 2010, "Another Look at the Identification of Dynamic Discrete Decision Processes: An Application to Retirement Behavior," "Journal of Business and Economic Statistics, 28, 201-218.

Altonji, J., T. Elder, and C. Taber, 2005a, "Selection on Observed and Unobserved Variables: Assessing the Effectiveness of Catholic Schools," Journal of Political Economy, Vol. 113.

Altonji, J., T. Elder, and C. Taber, 2005b, "An Evaluation of Instrumental Variable Strategies for Estimating the Effects of Catholic Schooling," Journal of Human Resources.

Angrist, J., and G. Imbens, 1999, "Comment on James J. Heckman, 'Instrumental Variables: A Study of Implicit Behavioral Assumptions Used in Making Program Evaluations"' The Journal of Human Resources, Vol. 34, No. 4, pp. 823-827.

Angrist, J., G. Imbens, and D. Rubin, 1996, "Identification of Causal Effects Using Instrumental Variables," Journal of the American Statistical Association, Vol. 91, June. 
Angrist, J., and S. Pischke, 2010, "The Credibility Revolution in Empirical Economics: How Better Research Design is Taking the Con out of Econometrics," NBER working paper 15794.

Bloom, H., L. Orr, S. Bell, G. Cave, F. Doolittle, W. Lin, and J.s Bos, 1997, "The Benefits and Costs of JTPA Title II-A Programs: Key Findings from the National Job Training Partnership Act Study," Journal of Human Resources, 32(3):549576.

Barlevy, G., 2008, "Identification of Search Models using Record Statistics," Review of Economic Studies, vol. 75(1), p29-64.

Blundell, R., A. Gosling, H. Ichimura, and C. Meghir, 2007, "Changes in the Distribution of Male and Female Wages Accounting for Employment Composition Using Bounds," Econometrica, 75, 323-363.

Buera, F., 2006, "Non-parametric Identification and Testable Implications of the Roy Model," unpublished manuscript, UCLA.

Burdett, K., and D. T. Mortensen, 1998, "Wage Differentials, Employer Size and Labor Market Equilibrium," International Economic Review, 39, 257-273.

Canals-Cerda, Jose, 2010, "Identification in Empirical Search Models when ages are Measured with Errors," unpublished manuscript, Federal Reserve Bank of Philadelphia.

Carneiro, P., J. Heckman and E. Vytlacil, 2010, "Estimating Marginal Returns to Education," unpublished manuscript, University College London.

Carneiro, P., and S. Lee, 2009, "Estimating Distributions of Potential Outcomes Using Local Instrumental Variables with an Application to Changes in College Enrollment and Wage Inequality," Journal of Econometrics, 149, 191-208.

Chamberlain, G., 1986, "Asymptotic Efficiency in Semiparametric Models with Censoring", Journal of Econometrics 32, 189-218.

Chen, X., 2007, "Large Sample Sieve Estimation of Semi-nonparametric Models," Handbook of Econometrics, North-Holland, chapt. 76. 
Das, M., W. Newey, and F. Vella, "Nonparametric Estimation of Sample Selection Models", The Review of Economic Studies, Vol. 70, No. 1 (Jan., 2003), pp. 33-58

Deaton, A., 2009, "Instruments of Development: Randomization in the Tropics, and the Search for the Elusive Keys to Economic Development," NBER working paper 14690.

Davidson, J., 1994, Stochastic Limit Theory, Oxford: Oxford University Press.

DiNardo, J., and D. Lee, "Program Evaluation and Research Designs", in Orley Ashenfelter and David Card, eds., Handbook of Labor Economics, Vol. 4, Elsevier Science, 2010.

Doyle, J., 2007, "Child Protection and Child Outcomes: Measuring the Effects of Foster Care", The American Economic Review. 97(5). December 2007: 15831610.

Eckstein, Z., and G van den Berg, 2007, "Empirical Labor Search: A Survey," Journal of Econometrics, 136, 531-564.

Elbers, C., and G. Ridder, 1982, "True and Spurious Duration Dependence: The Identifiability of the Proportional Hazard Model," Review of Economic Studies, 64, 403-409.

Evans, William N., and Robert M. Schwab, "Finishing High School and Starting College: Do Catholic Schools Make a Difference?" Quarterly Journal of Economics, 110 (1995), 947-974.

Flinn, C., and Heckman, J., 1982, "New Methods for Analyzing Structural Models of Labor Force Dynamics," Journal of Econometrics 18, 115-168.

French, E., and J. Song, 2010, " The Effect of Disability Insurance Receipt on Labor Supply," unpublished manuscript, Federal Reserve Bank of Chicago.

Heckman, J., 1979, "Sample Selection Bias as a Specification Error," Econometrica, 47 (1), 153-162.

Heckman, J.,1990, "Varieties of Selection Bias," American Economic Review, 80. 
Heckman, J., 1997, "Instrumental Variables: A Study of Implicit Behavioral Assumptions Used in Making Program Evaluations," The Journal of Human Resources, Vol. 32, No. 3 pp. 441-462.

Heckman, J., 1999, "Instrumental Variables: Response to Angrist and Imbens," The Journal of Human Resources, Vol. 34, No. 4, pp. 828-837.

Heckman, J., 2000, "Causal Parameters and Policy Analysis in Economics: A Twentieth Century Retrospective," Quarterly Journal of Economics, 115, 45-97.

Heckman, J. and B. Honoré, 1990, "The Empirical Content of the Roy Model," Econometrica, 58, 1121-1149.

Heckman, J. and B. Honoré, 1989, "The identifiability of the competing risks model," Biometrika 76, 32530 .

Heckman, J., R. LaLonde, and J. Smith, 1999, "The Economics and Econometrics of Active Labor Market Programs," in Ashenfelter and Card, Handbook of Labor Economics, vol. 3A, North-Holland, New York, 1865-2097.

Heckman, J., Lochner, L., and Taber, C., 1998, "Explaining Rising Wage Inequality: Explorations with a Dynamic General Equilibrium Model of Labor Earnings with Heterogeneous Agents," Review of Economic Dynamics.

Heckman, J., and S. Navarro, 2007, "Dynamic Discrete Choice and Dynamic Treatment Effects," Journal of Econometrics, 136, 341-396.

Heckman, J., and R. Robb, 1986, "Alternative Methods for Evaluating the Impact of Interventions," Longitudinal Analysis of Labor Market Data, Heckman and Singer eds., Cambridge University Press, New York, 156-245.

Heckman, J., and B. Singer, 1984a, "The Identifiability of the Proportional Hazard Model," Review of Economic Studies, 51, 231-241.

Heckman, J., and B. Singer, 1984b, "A Method for Minimizing the Impact of Distributional Assumptions in Econometric Models for Duration Data," Econometrica, $52,271-320$. 
Heckman, J. and C. Taber, 1994, "Econometric Mixture models and More General Models for Unobservables in Duration Analysis, Statistical Methods in Medical Research, Vol. 3 No. 3, 279-299.

Heckman, J. and C. Taber, 2008, "Roy model." The New Palgrave Dictionary of Economics Second Edition, Eds. Durlauf and Blume, Palgrave Macmillan.

Heckman, J., and S. Urzua, "Comparing IV with Structural Models: What Simple IV Can and Cannot Identify," forthcoming Journal of Econometrics.

Heckman, J. and E. Vytlacil, 1999, "Local Instrumental Variables and Latent Variable Models for Identifying and Bounding Treatment Effects," Proceedings of the National Academy of Sciences 96, 4730-4734.

Heckman, J. and E. Vytlacil, 2001, "Local instrumental variables," in Hsiao C., Morimune K. and Powell J. (eds), Nonlinear Statistical Inference: Essays in Honor of Takeshi Amemiya, Cambridge University Press, Cambridge, pp. 145.

Heckman, J. and E. Vytlacil, 2005, "Structural equations, treatment effects and econometric policy evaluation," Econometrica, Vol. 73, pp. 669738.

Heckman, J. and E. Vytlacil, 2007a, "Econometric Evaluation of Social Programs, Part I: Causal Models, Structural Models and Econometric Policy Evaluation," Handbook of Econometrics, Heckman and Leamer eds., North Holland, Amsterdam, 4779-4874.

Heckman, J. and E. Vytlacil, 2007b, "Econometric Evaluation of Social Programs, Part II: Using the Marginal Treatment Effect to Organize Alternative Econometric Estimators to Evaluate Social Programs, and to Forecast their Effects in New Environments," Handbook of Econometrics, Heckman and Leamer eds., North Holland, Amsterdam, 4875-5143.

Honoré, B., 1993, "Identification Results for Duration Models with Multiple Spells," Review of Economic Studies, 60, 241-246.

Hu, Y., and M. Shum, 2009, "Nonparametric Identification of Dynamic Models with Unobserved State Variables," Working Paper 543, Department of Economics, Johns Hopkins University. 
Ichimura, H., and Taber, C. (2002), "Semiparametric Reduced Form Estimation of Tuition Subsidies," American Economic Review , 286-2002.

Imbens, G., 2009, "Better LATE than Nothing: Some Comments on Deaton (2009) and Heckman and Urzua (2009)," unpublished manuscript, Harvard University.

Imbens, G., and Angrist, J., 1994, "Identification and Estimation of Local Average Treatment Effects," Econometrica, 62.

Imbens, G., and J. Wooldridge, 2009, "Recent Developments in the Econometrics of Program Evaluation," Journal of Economic Literature, 47:1, 5-86.

Kashara, H., and K. Shimotsu, 2009, "Nonparametric Identification of Finite Mixture Models of Dynamic Discrete Choice," Econometrica, 77(1), 135-175.

Klein, R., and F. Vella, 2010, "Estimation of a Class of Triangular Simultaneous Equations Models without Exclusion Restrictions, " Journal of Econometrics, 154, 154-164.

Keane, M., and Wolpin, K., 2001, "The Effect of Parental Transfers and Borrowing Constraints on Educational Attainment," (International Economic Review), 42, 1051-1103.

Keane, M., Todd, P., and K. Wolpin (2010) "The Structural Estimation of Behavioral Models: Discrete Choice Dynamic Programming Methods and Applications," in Orley Ashenfelter and David Card, eds., Handbook of Labor Economics, Vol. 4, Elsevier Science, 2010.

Lalonde, R., 1986, "Evaluating the Econometric Evaluations of Training Programs with Experimental Data," American Economic Review, 76, 604-620.

Leamer, E., 1983, "Let's Take the Con out of Econometrics," American Economic Review, 73(1): 31-43.

Lee, L-F, 1978, "Unionism and Wage Rates: A Simultaneous Equations Model with Qualitative and Limited Dependent Variables," International Economic Review, 19 (2), 415-433. 
Lippman, S., and J. McCall, 1976, "The Economics of Job Search: A Survey, Part I," Economic Inquiry, 14, 155-189.

Magnac, T. and D. Thesmar, 2002, "Identifying Dynamic Discrete Decision Processes," Econometrica, Vol. 70 No. 2, 801-816.

Manski, C., 1989, "Anatomy of the Selection Problem," The Journal of Human Resources, Vol. 24, No. 3, 343-360.

Manski, C., 1990, "Nonparametric Bounds on Treatment Effects, " American Economic Review, Vol. 80, No. 2, 319-323.

Manski, C., 1995, Identification Problems in the Social Sciences, Cambridge Mass.: Harvard University Press.

Manski, C., 1997, "Monotone Treatment Response," Econometrica, Vol. 65, No. 6, 1311-1334.

Manski, C., and J. Pepper, 2000, "Monotone Instrumental Variables with an Application to the Returns to Schooling," Econometrica, Vol. 68, No. 4, 997-1010.

Manski, C., and J. Pepper, 2009, "More on Monotone Instrumental Variables", The Econometric Journal, Vol. 12 Iss. s1, s200-s216.

Matzkin, R., 1992, Nonparametric and distribution-free estimation of the threshold crossing and binary Choice Model, Econometrica, 60, 239-270.

Matzkin, R., 1993, "Nonparametric Identification and Estimation of Polychotomous Choice Models," Journal of Econometrics, 58, 137-168.

Matzkin, R., 2007, "Nonparametric Identification," Handbook of Econometrics, Heckman and Leamer eds., North-Holland, Amsterdam, 5145-5368.

Mortensen, D., and C. Pissarides, 1994, "Job Creation and Job Destruction in the Theory of Unemployment," Review of Economic Studies, 61,397-415.

Neal, D., "The Effects of Catholic Secondary Schooling on Educational Attainment," Journal of Labor Economics 15 (1997), 98-123. 
Neal, D., and J. Grogger, 2000, "Further Evidence on the Effects of Catholic Secondary Schooling," Brookings-Wharton Papers on Urban Affairs, 151-193.

Postel-Vinay, F. and J.-M. Robin, 2002, "Wage Dispersion with Worker and Employer Heterogeneity", Econometrica, 70(6), 2295-350.

Ridder, G., 1990, The Non-parametric Identification of Generalized Accelerated FailureTime Models," Review of Economic Studies, 57, 167-182.

Rosenzweig, M., and K. Wolpin, 2000, "Natural 'Natural Experiments' in Economics," Journal of Economic Literature, 38(4): 827-874.

Roy, A.D., 1951, "Some Thoughts on the Distribution of Earnings," Oxford Economic Papers (New Series), 3, 135-146.

Rust, J., 1994, "Structural Estimation of Markov Decision Processes," forthcoming in R. Engle and D. McFadden eds., Handbook of Econometrics, Amsterdam, North Holland.

Shaikh, A., 2010, "Identification in Economics," Lecture Notes for Topics in Econometrics, http://home.uchicago.edu/ amshaikh/classes/topics_winter09.html, University of Chicago.

Sims, C., 2010, "Comment on Angrist and Pischke," unpublished manuscript, Princeton University.

Taber, C., 2000, "Semiparametric Identification and Heterogeneity in Dynamic Programming Discrete Choice Models," Journal of Econometrics.

Van den Berg, G., 2001, "Duration Models: Specification, Identification and Multiple Durations," in Heckman and Leamer eds. Handbook of Econometrics Volume 5, Elsevier.

Vytlacil, E., 2002, 'Independence, Monotonicity, and Latent Index Models: An Equivalence Result", Econometrica, Vol. 70, pp. 331341.

Willis, R. and Rosen, S., "Education and Self- Selection," Journal of Political Economy, 87(1979). 


\section{Working Paper Series}

A series of research studies on regional economic issues relating to the Seventh Federal Reserve District, and on financial and economic topics.

Risk Taking and the Quality of Informal Insurance: Gambling and Remittances in Thailand WP-07-01

Douglas L. Miller and Anna L. Paulson

Fast Micro and Slow Macro: Can Aggregation Explain the Persistence of Inflation?

WP-07-02

Filippo Altissimo, Benoît Mojon, and Paolo Zaffaroni

Assessing a Decade of Interstate Bank Branching

WP-07-03

Christian Johnson and Tara Rice

Debit Card and Cash Usage: A Cross-Country Analysis

Gene Amromin and Sujit Chakravorti

WP-07-04

The Age of Reason: Financial Decisions Over the Lifecycle

WP-07-05

Sumit Agarwal, John C. Driscoll, Xavier Gabaix, and David Laibson

Information Acquisition in Financial Markets: a Correction

WP-07-06

Gadi Barlevy and Pietro Veronesi

Monetary Policy, Output Composition and the Great Moderation

Benoît Mojon

Estate Taxation, Entrepreneurship, and Wealth

Marco Cagetti and Mariacristina De Nardi

WP-07-08

Conflict of Interest and Certification in the U.S. IPO Market

WP-07-09

Luca Benzoni and Carola Schenone

The Reaction of Consumer Spending and Debt to Tax Rebates -

Evidence from Consumer Credit Data

WP-07-10

Sumit Agarwal, Chunlin Liu, and Nicholas S. Souleles

Portfolio Choice over the Life-Cycle when the Stock and Labor Markets are Cointegrated Luca Benzoni, Pierre Collin-Dufresne, and Robert S. Goldstein

WP-07-11

Nonparametric Analysis of Intergenerational Income Mobility

WP-07-12 with Application to the United States

Debopam Bhattacharya and Bhashkar Mazumder

How the Credit Channel Works: Differentiating the Bank Lending Channel

WP-07-13 and the Balance Sheet Channel

Lamont K. Black and Richard J. Rosen

Labor Market Transitions and Self-Employment

WP-07-14

Ellen R. Rissman

First-Time Home Buyers and Residential Investment Volatility

WP-07-15

Jonas D.M. Fisher and Martin Gervais 


\section{Working Paper Series (continued)}

Establishments Dynamics and Matching Frictions in Classical Competitive Equilibrium

WP-07-16 Marcelo Veracierto

Technology's Edge: The Educational Benefits of Computer-Aided Instruction

WP-07-17

Lisa Barrow, Lisa Markman, and Cecilia Elena Rouse

The Widow's Offering: Inheritance, Family Structure, and the Charitable Gifts of Women Leslie McGranahan

WP-07-18

Incomplete Information and the Timing to Adjust Labor: Evidence from the

Lead-Lag Relationship between Temporary Help Employment and Permanent Employment Sainan Jin, Yukako Ono, and Qinghua Zhang

A Conversation with 590 Nascent Entrepreneurs

Jeffrey R. Campbell and Mariacristina De Nardi

WP-07-20

Cyclical Dumping and US Antidumping Protection: 1980-2001

WP-07-21

Meredith A. Crowley

Health Capital and the Prenatal Environment:

The Effect of Maternal Fasting During Pregnancy

Douglas Almond and Bhashkar Mazumder

WP-07-22

The Spending and Debt Response to Minimum Wage Hikes

WP-07-23

Daniel Aaronson, Sumit Agarwal, and Eric French

The Impact of Mexican Immigrants on U.S. Wage Structure

WP-07-24

Maude Toussaint-Comeau

A Leverage-based Model of Speculative Bubbles

WP-08-01

Gadi Barlevy

Displacement, Asymmetric Information and Heterogeneous Human Capital

WP-08-02

Luojia Hu and Christopher Taber

BankCaR (Bank Capital-at-Risk): A credit risk model for US commercial bank charge-offs Jon Frye and Eduard Pelz

WP-08-03

Bank Lending, Financing Constraints and SME Investment

Santiago Carbó-Valverde, Francisco Rodríguez-Fernández, and Gregory F. Udell

WP-08-04

Global Inflation

WP-08-05

Matteo Ciccarelli and Benoît Mojon

Scale and the Origins of Structural Change

WP-08-06

Francisco J. Buera and Joseph P. Kaboski

Inventories, Lumpy Trade, and Large Devaluations

WP-08-07

George Alessandria, Joseph P. Kaboski, and Virgiliu Midrigan 


\section{Working Paper Series (continued)}

School Vouchers and Student Achievement: Recent Evidence, Remaining Questions

WP-08-08

Cecilia Elena Rouse and Lisa Barrow

Does It Pay to Read Your Junk Mail? Evidence of the Effect of Advertising on

Home Equity Credit Choices

WP-08-09

Sumit Agarwal and Brent W. Ambrose

The Choice between Arm's-Length and Relationship Debt: Evidence from eLoans

Sumit Agarwal and Robert Hauswald

WP-08-10

Consumer Choice and Merchant Acceptance of Payment Media

WP-08-11

Wilko Bolt and Sujit Chakravorti

Investment Shocks and Business Cycles

Alejandro Justiniano, Giorgio E. Primiceri, and Andrea Tambalotti

WP-08-12

New Vehicle Characteristics and the Cost of the

Corporate Average Fuel Economy Standard

WP-08-13

Thomas Klier and Joshua Linn

Realized Volatility

WP-08-14

Torben G. Andersen and Luca Benzoni

Revenue Bubbles and Structural Deficits: What's a state to do?

WP-08-15

Richard Mattoon and Leslie McGranahan

The role of lenders in the home price boom

WP-08-16

Richard J. Rosen

Bank Crises and Investor Confidence

WP-08-17

Una Okonkwo Osili and Anna Paulson

WP-08-18

Life Expectancy and Old Age Savings

Mariacristina De Nardi, Eric French, and John Bailey Jones

Remittance Behavior among New U.S. Immigrants

WP-08-19

Katherine Meckel

Birth Cohort and the Black-White Achievement Gap:

The Roles of Access and Health Soon After Birth

WP-08-20

Kenneth Y. Chay, Jonathan Guryan, and Bhashkar Mazumder

Public Investment and Budget Rules for State vs. Local Governments

WP-08-21 Marco Bassetto

Why Has Home Ownership Fallen Among the Young?

WP-09-01

Jonas D.M. Fisher and Martin Gervais

Why do the Elderly Save? The Role of Medical Expenses

WP-09-02

Mariacristina De Nardi, Eric French, and John Bailey Jones 


\section{Working Paper Series (continued)}

Using Stock Returns to Identify Government Spending Shocks

WP-09-03

Jonas D.M. Fisher and Ryan Peters

Stochastic Volatility

Torben G. Andersen and Luca Benzoni

WP-09-04

The Effect of Disability Insurance Receipt on Labor Supply

WP-09-05

Eric French and Jae Song

CEO Overconfidence and Dividend Policy

WP-09-06

Sanjay Deshmukh, Anand M. Goel, and Keith M. Howe

Do Financial Counseling Mandates Improve Mortgage Choice and Performance?

WP-09-07

Evidence from a Legislative Experiment

Sumit Agarwal,Gene Amromin, Itzhak Ben-David, Souphala Chomsisengphet, and Douglas D. Evanoff

Perverse Incentives at the Banks? Evidence from a Natural Experiment

WP-09-08

Sumit Agarwal and Faye H. Wang

Pay for Percentile

Gadi Barlevy and Derek Neal

WP-09-09

The Life and Times of Nicolas Dutot

WP-09-10

François R. Velde

Regulating Two-Sided Markets: An Empirical Investigation

Santiago Carbó Valverde, Sujit Chakravorti, and Francisco Rodriguez Fernandez

WP-09-11

The Case of the Undying Debt

WP-09-12

François R. Velde

Paying for Performance: The Education Impacts of a Community College Scholarship Program for Low-income Adults

Lisa Barrow, Lashawn Richburg-Hayes, Cecilia Elena Rouse, and Thomas Brock

WP-09-13

Establishments Dynamics, Vacancies and Unemployment: A Neoclassical Synthesis

WP-09-14

Marcelo Veracierto

The Price of Gasoline and the Demand for Fuel Economy:

Evidence from Monthly New Vehicles Sales Data

WP-09-15

Thomas Klier and Joshua Linn

Estimation of a Transformation Model with Truncation, Interval Observation and Time-Varying Covariates

WP-09-16

Bo E. Honoré and Luojia Hu

Self-Enforcing Trade Agreements: Evidence from Antidumping Policy

WP-09-17

Chad P. Bown and Meredith A. Crowley

Too much right can make a wrong: Setting the stage for the financial crisis

WP-09-18 Richard J. Rosen 


\section{Working Paper Series (continued)}

Can Structural Small Open Economy Models Account

for the Influence of Foreign Disturbances?

WP-09-19

Alejandro Justiniano and Bruce Preston

Liquidity Constraints of the Middle Class

WP-09-20

Jeffrey R. Campbell and Zvi Hercowitz

Monetary Policy and Uncertainty in an Empirical Small Open Economy Model

WP-09-21

Alejandro Justiniano and Bruce Preston

Firm boundaries and buyer-supplier match in market transaction:

IT system procurement of U.S. credit unions

WP-09-22

Yukako Ono and Junichi Suzuki

Health and the Savings of Insured Versus Uninsured, Working-Age Households in the U.S.

WP-09-23

Maude Toussaint-Comeau and Jonathan Hartley

The Economics of “Radiator Springs:” Industry Dynamics, Sunk Costs, and

Spatial Demand Shifts

Jeffrey R. Campbell and Thomas N. Hubbard

WP-09-24

On the Relationship between Mobility, Population Growth, and

Capital Spending in the United States

Marco Bassetto and Leslie McGranahan

WP-09-25

The Impact of Rosenwald Schools on Black Achievement

WP-09-26

Daniel Aaronson and Bhashkar Mazumder

Comment on "Letting Different Views about Business Cycles Compete"

WP-10-01

Jonas D.M. Fisher

Macroeconomic Implications of Agglomeration

WP-10-02

Morris A. Davis, Jonas D.M. Fisher and Toni M. Whited

Accounting for non-annuitization

Svetlana Pashchenko

WP-10-03

Robustness and Macroeconomic Policy

WP-10-04

Gadi Barlevy

Benefits of Relationship Banking: Evidence from Consumer Credit Markets

Sumit Agarwal, Souphala Chomsisengphet, Chunlin Liu, and Nicholas S. Souleles

WP-10-05

The Effect of Sales Tax Holidays on Household Consumption Patterns

WP-10-06

Nathan Marwell and Leslie McGranahan

Gathering Insights on the Forest from the Trees: A New Metric for Financial Conditions

WP-10-07

Scott Brave and R. Andrew Butters

Identification of Models of the Labor Market

WP-10-08

Eric French and Christopher Taber 